\title{
REGULATION OF ANTIOXIDANT DEFENSES, DNA DAMAGE REPAIR, THE IMMUNE RESPONSE, AND NEUROPROTECTION DURING HIBERNATION IN THE THIRTEEN-LINED GROUND SQUIRREL
}

\author{
Kama E. Szereszewski
}

Ph.D. Carleton University, 2019

A thesis submitted to the Faculty of Graduate Studies and Research in partial fulfillment

of the requirements for the degree of

\author{
Doctor of Philosophy \\ Department Biology \\ Carleton University \\ Ottawa, Ontario, Canada \\ (C) Copyright 2019
}

Kama E. Szereszewski 
The undersigned hereby recommend to the Faculty of Graduate Studies and Research acceptance of this thesis

"REGULATION OF ANTIOXIDANT DEFENSES, DNA DAMAGE REPAIR, THE IMMUNE RESPONSE, AND NEUROPROTECTION DURING HIBERNATION IN THE THIRTEEN-LINED GROUND SQUIRREL

"

\begin{abstract}
Submitted by
Kama Elena Szereszewski, M. Sc.

In partial fulfillment of the requirements for the degree of Doctor of Philosophy in Biology with Combined Specialization in Biochemistry
\end{abstract}

Chair, Department of Biology

Thesis Supervisor

External Examiner 


\begin{abstract}
Hibernation is a fascinating survival adaptation that allows animals to transition into a torpid state to survive the winter by coordinating a strong suppression of metabolic rate, conservation of fuel/energy, and reduction of body temperature. This strategy permits thirteen-lined ground squirrels (Ictidomys tridecemlineatus) and other hibernating mammals to endure the harsh winter season when there is little access to food. Many energy-expensive cellular processes are suppressed, including gene transcription and protein synthesis/turnover, but are reactivated rapidly when animals arouse back to euthermia. Both torpor and arousal can have damaging consequences; for example, during arousal, reactive oxygen species flood the cell causing oxidative damage to numerous cellular components. Therefore, hibernation requires many pro-survival mechanisms to mitigate multiple types of damage: e.g. from oxidative damage, DNA damage, and pathogen attack, among others. The research reported in this thesis on damage control processes in hibernators shows that antioxidant enzymes such as PRDXs are upregulated in key tissues but in an isoform-specific and time-specific manner over the torpor-arousal cycle. PRDX2, 3, 4 and 6 were found to be significantly upregulated in specific tissues. Similarly, DNA damage repair is initiated during torpor and is characterized by the binding of repair proteins such as Ku80 and the MRN complex to the site of breaks, but ligation (with XLF) reactions to fully repair DNA do not appear to occur until the arousal period. Pro-inflammatory mechanisms are also used to deal with pathogens; these remain active at basal levels in a tissue-specific manner during torpor, but are up-regulated in the final stages just before arousal or only during arousal depending on the tissue, such as the induction of CCL5, a recruiter of monocytes. A cyto/neuro-protective mitochondrial peptide, shumanin, was also identified that is induced in a tissue-specific manner, helping to protect key organs such as the brain cortex and adipose tissues. The results show that hibernation is a complex, multi-faceted process that employs specific adaptations of damage prevention/repair pathways to protect squirrel tissues from damage not only during prolonged torpor but over the transitional states to/from torpor and does so expertly while conserving energy until such a time that repair mechanisms may be fully initiated.
\end{abstract}




\section{Acknowledgements}

I would first like to thank my supervisor Dr. Kenneth B. Storey for pushing me beyond the limits I believed I had and giving me the opportunity to maximize my learning experience. I have learned so much from you in both a scientific and personal capacity and believe that I have grown so much in the past several years in your lab, and have a lot more to go. I am eternally grateful for all that you have done for me. Also, I would like to give immense thanks to Jan, who has contributed countless hours reviewing and editing manuscripts, theses and posters as well as acting as a personal mentor. Jan, I would not have been able to survive without you and I cannot thank you enough for all that you have done for me.

I would like to thank my family for their support throughout my studies, for their patience and understanding as I spent many weekends in the lab. A special thanks to my inlaws who have always been supportive and loving, especially in the last few years when I needed it most. A special thank you to my husband Avery who I am sure is more than happy that I am finally done with my studies. Thank you for loving me, supporting me, meal prepping for me and dealing with my nonsense for the past 7 years. You have been there from day 1 of this journey and I will never forget your love and support.

A huge thanks to my friends, who have been there for me always, often treating me as real family, and have supported me and been there for me through thick and thin. Jackie, Himali, May, Annemarie, Mateja, Hanane I will never forget the scientific conversations, all the laughs, sleepovers, dinners, and so much more. You have been strong supporters in my life. I know many of you will never read this thesis, but know that you have meant more to me than words can say. Finally, a very special thank you to those with whom I have grown close in the last several months; Anna, Spencer, and especially Evan. You have my eternal gratitude for everything you have done for me and will always have a place in my heart. I couldn't have gotten through these months without you. All my love. 


\section{Table of Contents}

Title page $\quad$ i

Acceptance Sheet $\quad$ ii

$\begin{array}{lll}\text { Abstract } & \text { iii }\end{array}$

Acknowledgements

Table of Contents $\quad$ V

List of Abbreviations $\quad$ vi

List of Figures vii

List of Appendices $\quad \mathrm{x}$

Chapter 1: General Introduction 1

Chapter 2: Peroxiredoxin (oxidative stress) 19

Chapter 3: PARP (DNA damage) 43

Chapter 4: IRF3 transcription factor (immune response) 65

Chapter 5: s-humanin peptide (squirrelin) 96

$\begin{array}{ll}\text { Chapter 6: General Discussion } & 117\end{array}$

References 133 


\section{List of Abbreviations}

\begin{tabular}{|c|c|}
\hline APS & Ammonium persulfate \\
\hline DTT & Dithiolthreitol \\
\hline $\mathrm{EtBr}$ & Ethidium Bromide \\
\hline EDTA & Ethylenediamine tetraacetic acid \\
\hline HRP & Horseradish peroxidase \\
\hline PMSF & Phenylmethanesulfonylfluoride \\
\hline SDS & Sodium dodecyl sulfate \\
\hline TEMED & $\mathrm{N}, \mathrm{N}, \mathrm{N}$ ', N'-tetramethylethylenediamine \\
\hline TRIS & Tris(hydroxymethyl)aminomethane \\
\hline mRNA & messenger RNA \\
\hline ATP & Adenosine triphosphate \\
\hline PAGE & Polyacrylamide gel electrophoresis \\
\hline PCR & Polymerase chain reaction \\
\hline PVDF & Polyvinylidine fluoride \\
\hline $\mathrm{EC}$ & Euthermic control \\
\hline EN & Entrance (into torpor) \\
\hline $\mathrm{LT}$ & Late torpor \\
\hline EA & Early arousal \\
\hline IA & Interbout arousal \\
\hline $\mathrm{AOE}$ & Antioxidant enzyme \\
\hline PRDX & Peroxiredoxin \\
\hline ROS & Reactive oxygen species \\
\hline SOD & Superoxide dismutase \\
\hline $8-\mathrm{OH}-\mathrm{dG}$ & 8-hydroxy-2'-deoxyguanosine \\
\hline NHEJ & Non-homologous end joining \\
\hline HR & Homologous recombination \\
\hline
\end{tabular}




\section{List of Figures}

Figure 1.1: Body temperature $(\mathrm{Tb})$ of a thirteen-lined ground squirrel as a function of time over one hibernation season.

Figure 2.1: Relative antioxidant capacity, in the liver and brown adipose tissue across the torpor-arousal cycle.

Figure 2.2: Relative protein expression of the antioxidant enzymes SOD1, CAT, SOD2, TRX1, and PRX2 in the liver of I. tridecemlineatus in control versus late torpor.

Figure 2.3: Relative protein expression of peroxiredoxin isoforms 1-4 and 6 in the liver of I. tridecemlineatus throughout the torpor-arousal cycle.

Figure 2.4: Relative protein expression of peroxiredoxin isoforms 1-4,6 in the brown adipose tissue of $I$. tridecemlineatus throughout the torpor-arousal cycle.

Figure 2.5: Relative protein expression of peroxiredoxin isoforms 1-4 and 6 in white adipose tissue of I. tridecemlineatus throughout the torpor-arousal cycle.

Figure 3.1. Relative abundance of 8-hydroxy-2'-deoxyguanosine in liver and skeletal muscle of Ictidomys tridecemlineatus during late torpor (LT) and early arousal (EA) as compared with euthermic controls (EC).

Figure 3.2. Relative protein expression levels of the ATM and ATR kinases in liver and skeletal muscle of Ictidomys tridecemlineatus during torpor.

Figure 3.3. Relative protein expression levels of cytoplasmic and nuclear PARP-1 in liver (A) and skeletal muscle (B) of Ictidomys tridecemlineatus during torpor.

Figure 3.4. Relative protein expression levels of DNA damage repair targets Ku80, XLF, RAD50, p-P95, Mre11, and (total) PARP-1 in liver (A) and skeletal muscle (B) of Ictidomys tridecemlineatus during torpor.

Figure 4.1: Histogram of normalized $I R F 3$ relative gene transcript levels across the torporarousal cycle in liver (LIV), brown adipose tissue (BAT) and white adipose tissue (WAT).

Figure 4.2: Relative protein expression levels of total IRF3 and phosphorylated IRF (Ser396) in Ictidomys tridecemlineatus liver tissue across the torpor-arousal cycle.

Figure 4.3: Relative protein expression levels of upstream IRF3 targets in Ictidomys tridecemlineatus liver tissue throughout the torpor-arousal cycle.

Figure 4.4: Relative protein expression levels of total IRF3 and phosphorylated IRF (Ser396) in Ictidomys tridecemlineatus brown adipose tissue throughout the torpor-arousal cycle.

Figure 4.5: Relative protein expression levels of upstream IRF3 targets in Ictidomys tridecemlineatus brown adipose tissue throughout the torpor-arousal cycle. 
Figure 4.6: Relative protein expression levels of total IRF3 and phosphorylated IRF (Ser396) in Ictidomys tridecemlineatus white adipose tissue throughout the torpor-arousal cycle.

Figure 4.7: Relative protein expression levels of upstream IRF3 targets in Ictidomys tridecemlineatus white adipose tissue throughout the torpor-arousal cycle.

Figure 4.8: Histogram of normalized IRF3 downstream gene transcript levels in Ictidomys tridecemlineatus liver tissue throughout the torpor-arousal cycle.

Figure 4.9: Histogram of normalized IRF3 downstream gene transcript levels in Ictidomys tridecemlineatus brown adipose tissue throughout the torpor-arousal cycle.

Figure 4.10: Histogram of normalized IRF3 downstream gene transcript levels in Ictidomys tridecemlineatus white adipose tissue throughout the torpor-arousal cycle. Data are mean critical threshold $(\mathrm{Ct})$ ( \pm S.E.M. $n=4$ independent trials on tissue from different animals). Values that share the same letter are not significantly different $(p<0.05)$.

Fig. 5.1. Multiple sequence alignment of the predicted homologues of humanin peptide encoded by MT-RNR2 and MT-RNR2-like mitochondrial and nuclear genes in several mammalian species.

Fig. 5.2. Homology modeling of humanin (A) and predicted S-humanin (B) performed using the I-Tasser program with the human PDB code as the primary template.

Fig. 5.3. Histogram of standardized s-humanin gene transcript levels across the torporarousal cycle in liver (LIV), skeletal muscle (MUS), brown adipose tissue (BAT) and white adipose tissue (WAT).

Fig. 5.4. Histogram of standardized s-humanin relative transcript levels under EC and LT conditions in brain cortex (BCTX) and brainstem (BSTEM).

Fig. 5.5. Histogram of standardized s-humanin relative protein levels in control and late torpor.

\section{List of Appendices}

Appendix 2.1. Luminex multiplex analysis

Appendix 4.1. Primer design for RT-qPCR

Appendix 4.2. IRF3 pathway 


\section{CHAPTER 1}

\section{GENERAL INTRODUCTION}




\section{General Introduction}

Many animals endure oxidative stress when faced with harsh environmental conditions such as decreasing temperatures during winter. In hibernating squirrels, oxygen consumption falls to $\sim 2 \%$ of basal levels, but then rises to $\sim 300 \%$ during periods of arousal [1]. The increase in metabolic rate sets up the conditions for over-generation of ROS, therefore, protective pathways must be induced to protect the cell from radical-induced damage. Examples of key processes include antioxidant defenses, which must be induced to mitigate accumulating levels of ROS and pose a significant threat to the cell damaging proteins, lipids and DNA. Thus, DNA repair mechanisms must also be upregulated to protect it from oxidative damage such as lesions, adduct formation and base modifications. The immune response must be induced to protect not only against invading pathogens but free radicals themselves which could stimulate a pro-inflammatory environment. Finally, protective peptides are also induced to protect tissues by inducing pro-survival pathways and halting cell death. As such, the aim of this study is to examine these mechanisms in the context of hibernation to examine how the thirteen-lined ground squirrel can adapt to the oxidative environment.

Oxygen

Oxygen, one of the most abundant elements in the atmosphere, first described as the "food of life" element in 1604 [2] was pivotal to the development of living systems. The incorporation of oxygen into living systems began around 2.5 billion years ago with the evolution of organisms capable of transducing solar energy and converting it into the chemical energy of carbon bonds [3]. This process, known as photosynthesis, allowed for the generation of glucose from carbon dioxide and water, with diatomic $\mathrm{O}_{2}$ generated as a side product. As photosynthetic organisms prospered, so did the increase in the concentration of atmospheric $\mathrm{O}_{2}$. Then, approximately 1.5 billion years ago, eukaryotic organisms appeared containing subcellular organelles including mitochondria, capable of fully oxidizing glucose back to $\mathrm{CO}_{2}$ and $\mathrm{H}_{2} \mathrm{O}$. The process, referred to as cellular respiration, occurs in the mitochondrial matrix. Here, reducing agents pass through the 
mitochondrial respiratory complex and result in the formation of a proton gradient used to drive the oxidative phosphorylation to produce adenosine 5'-triphosphate (ATP) [3].

The evolution of metazoans resulted in the ability to recover the energy contained within the chemical bonds of glucose through oxidative phosphorylation. This process, which is 18 times more efficient at producing ATP per mole of glucose than glycolysis, provides the necessary energy for developing and maintaining complex multicellular organisms. However, the utilization of oxygen as a substrate for ATP production is not without risk, as a portion of the electrons transferred through the mitochondrial respiratory chain will not react with $\mathrm{O}_{2}$ to form water. Approximately $5 \%$ of these electrons escape and combine with oxygen to result in the generation of reactive intermediates known as free radicals and reactive oxygen species (ROS). The intermediates are partially reduced forms of atmospheric oxygen $\left(\mathrm{O}_{2}\right)$ that typically result from the excitation of $\mathrm{O}_{2}$ to form singlet oxygen $\left(\mathrm{O}_{2}{ }^{1}\right)$ from the transfer of one, two or three electrons to $\mathrm{O}_{2}$ to form, respectively, a superoxide radical $\left(\mathrm{O}_{2}^{-}\right)$, hydrogen peroxide $\left(\mathrm{H}_{2} \mathrm{O}_{2}\right)$ or a hydroxyl radical $\left(\mathrm{HO}^{-}\right)[4,5]$. The accumulation of these reactive oxygen species in cells is capable of causing extensive damage to macromolecules and its effects must therefore be mitigated.

\section{Reactive oxygen species}

Reactive oxygen species can be derived from many sources including endogenous (mitochondria, peroxisomes, lipoxygenases, NADPH oxidase, cytochrome p450) or exogenous inputs (UV, ionizing radiation, chemotherapeutics, inflammatory cytokines, environmental toxins). They can lead to impaired physiological function, a shift in the homeostasis of normal growth and development, as well as cause damage to signaling pathways [6-8]. It was suggested in the 1950s by Denham Harman, that endogenous free radicals were responsible for the "free-radical theory" of ageing and that toxic radicals generated in cells resulted in a pattern of cumulative damage [9]. However, ROS have since been shown to play important roles in diverse physiological processes including neurotransmission, blood pressure regulation, cellular defense mechanisms, smooth muscle relaxation, and immune regulation [4,5]. Increasing levels of ROS above homeostasis, however, have the potential to cause severe oxidative stress and damage macromolecules like lipids, proteins, mitochondrial and nuclear DNA [10]. 
Mitochondria produce the bulk of the ATP energy in the cell, and correspondingly consume the bulk of intracellular oxygen. The free-radical theory of aging is now often thought to be synonymous with the rate-of living hypothesis, where the higher the metabolic rate of an organism, the greater the production of ROS, and hence, the shorter the life span. However, recent studies in various species have found that this correlation is not always maintained, and is in fact contradicted. This is especially true for birds and primates which tend to live longer than would be expected given their metabolic rates. However, analyses of their oxidant production rates have demonstrated that these species tend to produce fewer ROS and therefore, it is likely that ROS production, rather than metabolic rate, provides the strongest correlation with overall longevity. Interestingly, this theory is also contradicted in studies examining the naked mole rat, the longest-living rodent, capable of surviving upwards of 30 years and yet, accumulates significantly elevated levels of ROS [11].

ROS-induced oxidation of proteins can lead to a change in the three-dimensional structure of that protein as well as fragmentation, aggregation or cross-linking. It can also make the protein more susceptible to degradation. ROS are also a major source of DNA damage, causing both double and single stranded breaks, removal of nucleotides, and a variety of modifications of the organic bases which can cause permanent changes, deleterious mutations, or loss of gene expression [12]. ROS are also capable of causing damage to the lipids of cell membranes whereby the bonds between fatty acids become prone to damage or abstraction. Lipid peroxidation may result in a self-perpetuating process as certain radicals are both reaction initiators as well as the products of lipid peroxidation and will attack lipid membranes thus altering membrane fluidity, permeability and could affect cellular metabolic functions as well [8]. Fortunately, numerous protective mechanisms exist to mitigate this damage and one such model of oxidative-stress tolerance is mammalian hibernation.

\section{Hibernation}

The ability to hibernate is not an adaptation that is unique to ground squirrels or bears. In fact, this hypometabolic state occurs widely across the Class Mammalia including mouse opossums, little brown bats, hedgehogs, tenrecs, marsupials, lemurs, and other mammals 
$[13,14]$. Hibernation is an adaptation to limited or complete lack of food availability and decrease in ambient temperatures to below freezing. To cope with these conditions most hibernating species, including ground squirrels abandon homeothermy in favour of heterothermy, and suppress their body temperatures to near-ambient, often as low as $0-5^{\circ} \mathrm{C}$, whereas other mammalian hibernators, chiefly bears, maintain relatively high body temperatures during torpor $\left(\sim 25-33^{\circ} \mathrm{C}\right)[14,15]$. They then return to euthermia without any ill effects. Additionally, many hibernators use body fuel reserves to survive the winter relying on a period of hyperphagia prior to the hibernation that packs on fat stores, sometimes nearly doubling body mass [16]. As a result, most species, including ground squirrels use triglyceride lipids (and beta-oxidation) as their main fuel/energy source during torpor, which also allows for carbohydrate sparing [13, 16, 17]. Incidentally, the fatty acid composition of stored lipids can also affect the depth and duration of the hibernation period [16]. Hibernation consists of multiple bouts of deep torpor (lasting days to weeks), punctuated by periodic arousals (lasting several hours) during which the animals rewarm back to euthermic body temperatures (Figure 1.1). During these arousals, the brown adipose tissue plays a critical role in uncoupling fuel oxidation from ATP production resulting in non-shivering thermogenesis $[13,16,18]$. During torpor, several mechanisms assist in the reduction of ATP expensive processes and involve important molecular "switches" that suppress these metabolic pathways. For example, gene transcription, protein synthesis, and ATP-dependent transmembrane pumps are suppressed during torpor $[19,20]$. Equally as important, is the upregulation of pro-survival mechanisms that protect against the deleterious effects that accompany ischemia-reperfusion and hypothermia [13, $20]$.

The reduction in metabolic rate during torpor is quite remarkable. As oxygen consumption at body temperatures approaching $0^{\circ} \mathrm{C}$ is typically around $1-5 \%$ of euthermic values [17], hibernation generally provides an energy savings of 90-95\% of energy expenditures compared with the euthermic state $[13,18,19,21]$. This is accompanied by several physiological changes (for ground squirrels) including a significant decrease in breathing rates (from 40 breaths per minute to less than 1), heart rate (from 350-400 beats per minute to 5-10), and tissue perfusion rates (down to $10 \%$ of euthermic). These and other physiological parameters are rapidly reversed upon exit from torpor during intermittent 
arousals, where a 10-20-fold surge in oxygen consumption occurs as the squirrel rewarms to $37^{\circ} \mathrm{C}[17,22]$. It is this surge of oxygen that brings with it an increase of ROS requiring increased antioxidant defenses to protect macromolecules from damage during extended periods of torpor and arousal [23]. However, the hibernating ground squirrel is evolutionarily equipped with various pro-survival mechanisms to protect its DNA, proteins, and lipids from oxidative stress-induced damage.

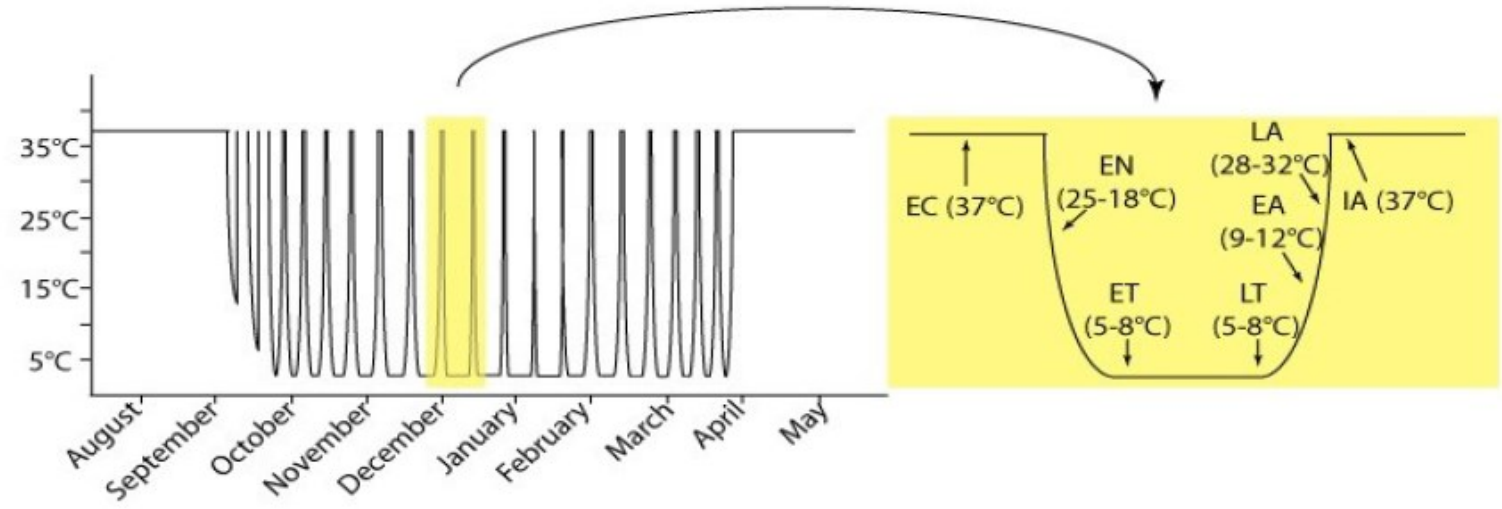

Figure 1.1: Body temperature $(\mathrm{Tb})$ of a thirteen-lined ground squirrel as a function of time over one hibernation season. Highlighted in yellow is one torpor-arousal cycle. The different stages are: (1) euthermic in the cold room (EC), (2) entrance into torpor (EN), (3) early torpor (ET), (4) late torpor (LT), (5) early arousal (EA), and (6) interbout arousal (IA). Modified from Hindle et al. 2011.

\section{Antioxidant enzymes}

Uncontrolled production of ROS often leads to damage of cellular macromolecules, but fortunately, several protective mechanisms exist to neutralize them. Antioxidant enzymes suppress the production of reactive species by reducing hydroperoxides, sequestering metal ions, scavenging free radicals, and clearing the damage caused by these to the cell [10]. Some antioxidants also induce the expression or biosynthesis of other antioxidants, resulting in a stronger response. Many types of antioxidant enzymes exist; they can be endogenous enzymatic, non-enzymatic, metal binding proteins (e.g. ferritin, ceruloplasmin), and phytoconstituents and nutrients (e.g. vitamin E). They are also 
classified as "first/second/third line of defense" on the basis of whether they are radical preventive, radical scavenging or radical-induced damage repairing [24]. Antioxidants that function to suppress or prevent the formation of free radicals are the first-line defenders and work very quickly to neutralize any molecules with the potential of developing into free radicals, or free radicals with the ability to induce the production of others. Second line defense antioxidants are generally referred to as scavenging antioxidants. They scavenge active radicals and act to inhibit chain reactions. They neutralize free radicals by donating electrons, and in the process, become free radicals but with less damaging effects. They can then be detoxified by other members in this group including ascorbic acid, uric acid, glutathione, vitamin E, and ubiquinol [24]. Third line defense antioxidants generally mitigate the damage that has already occurred. These are enzymes which repair the damage caused by free-radicals that reconstitute the cell membrane, DNA, or proteins by recognizing, breaking down and removing oxidized or damaged products and preventing their accumulation. Common examples of these include the DNA repair enzyme systems and proteolytic enzymes.

Three key enzymes are at the top of the list of first line defenders: superoxide dismutase (SOD), catalase (CAT) and glutathione peroxidase (GPx). SOD catalyses the dismutation of $\mathrm{O}_{2}{ }^{-}$into oxygen and $\mathrm{H}_{2} \mathrm{O}_{2}$. In mammals, there are three isoforms of SOD (SOD1 [CuZnSOD]; SOD2 [MnSOD]; and SOD3 [ecSOD]) [25]. Each SOD is a product of distinct gene and subcellular localization, but catalyzes the same reaction. The mechanism of dismutation of $\mathrm{O}_{2}{ }^{-}$to $\mathrm{H}_{2} \mathrm{O}_{2}$ by SOD involves alternate reduction and reoxidation of a redox active transition metal such as copper $(\mathrm{Cu})$ or manganese $(\mathrm{Mn})$ at the enzyme active site. Catalase, another first-line defense antioxidant, breaks down $\mathrm{H}_{2} \mathrm{O}_{2}$ into water and molecular oxygen. However, catalase is absent in the mitochondria, and therefore the reduction of $\mathrm{H}_{2} \mathrm{O}_{2}$ to water and lipid peroxides to their corresponding alcohols is generally carried out in this subcellular compartment by glutathione peroxidase[24]. CAT is present in almost all tissues that utilize oxygen. It uses either an iron or manganese catalytic metal, and reduces hydrogen peroxide, completing the detoxification process initiated by SOD [26]. CAT is highly efficient and breaks down millions of hydrogen peroxide molecules per second. GPx also breaks down $\mathrm{H}_{2} \mathrm{O}_{2}$ to water but mainly in the mitochondria and 
sometimes in the cytosol. There could be at least eight GPx enzymes in humans (GPx1-8), where GPx1 is the most abundant and is present in virtually all cells [24].

Two other important antioxidant enzymes are Thioredoxin (TRX) and Peroxiredoxin (PRDX). These have been shown to detoxify $\mathrm{H}_{2} \mathrm{O}_{2}$ as well as assist in its regulation as a signaling molecule of diverse physiological processes [27-29]. Studies have shown that (a) transient elevations of $\mathrm{H}_{2} \mathrm{O}_{2}$ occur in response to various cytokines and growth factors, (b) elevated $\mathrm{H}_{2} \mathrm{O}_{2}$ levels can affect the function of various protein kinases, phosphatases, transcription factors and $\mathrm{G}$ proteins, and (c) inhibition of $\mathrm{H}_{2} \mathrm{O}_{2}$ generation results in a complete blockage of signaling by various growth factors $[7,30,31]$. Therefore, a delicate balance must exist between the pathological and physiological levels of $\mathrm{H}_{2} \mathrm{O}_{2}$ where PRDX has been shown to play a crucial role.

The TRX system, is composed of NADPH-dependent thioredoxin reductase (TRXR). Mammalian cells possess two TRX systems - cytosolic TRX1 and mitochondrial TRX2 where TRX2 has only two cysteines in its active site, whereas TRX1 has three additional cysteines [32]. TRX provides electrons to thiol-dependent PRDXs to break down $\mathrm{H}_{2} \mathrm{O}_{2}$ in a ping-pong mechanism [33]. PRDXs are a ubiquitous family of antioxidant proteins with 6 different isoforms that use specialized cysteine residues to break down hydroperoxides. The cysteine in the active site of PRDX is very sensitive to oxidation, forming a sulfenic acid upon exposure to $\mathrm{H}_{2} \mathrm{O}_{2}$. A disulfide bond is formed resulting in an intermolecular

dimer which is reduced by TRX [7, 34]. Given the multi-faceted functions of peroxiredoxins and $\mathrm{H}_{2} \mathrm{O}_{2}$, measuring the expression of these enzymes has made them an interesting group of enzymes to study in a mammalian hibernator.

\section{DNA damage}

DNA, the basic unit of inheritance, is an intrinsically reactive molecule highly susceptible to chemical modifications by oxidative stress. Several enzymes exist to detect and repair mutations in the DNA sequence, however, some errors may go undetected, repair enzymes may make a mistake, or accumulating errors may overwhelm the cell and therefore initiate a stoppage of the cell cycle. Fortunately, cells are equipped with rigorous systems 
of DNA repair, damage tolerance, cell cycle checkpoints and cell death pathways that collectively function to reduce these deleterious consequences [35]. A robust DNA damage repair response exists in cells that allows sufficient time for DNA repair pathways to remove the damaged substrate quickly and efficiently. The major repair pathways include base excision repair (BER), nucleotide excision repair (NER), homologous recombination (HR) and non-homologous end joining (NHEJ) which are active throughout different stages of the cell cycle and are specific to the type of damage occurring [35]. If the damage is excessive, is unable to be repaired or persists, programmed cell death (apoptosis) is initiated to remove the damaged cells. Not surprisingly, the DNA damage response is dysregulated in various types of cancers where mutagenesis and genomic instability promote its progression [35-37]. Likewise, aging is attributed to the attrition of telomeric ends and the failing capacities of these pathways [35]. Although different types of DNA damage exist (e.g. base deamination, abasic sites, etc.), oxidative damage to DNA is an important source of damage for the hibernating squirrel. Although ROS by-products from cellular respiration can perform important cellular functions, in excess, they can cause hundreds of different oxidative base lesions. The reaction of these electrophilic radicals with DNA bases can cause modifications to hydrogen bonds, removal of hydrogen atoms from their methyl groups, or attack on sugar residues of DNA in their immediate vicinity [38]. As well, hydroxylation of bases is a common mutation, such as the modification of the C-8 guanine base in the saturated imidazole ring forming 8-hydroxy-deoxyguanosine [37, 39].

Fortunately, cells have an intricate DNA damage response system which is initiated within minutes of damage detection. The damage response is a highly coordinated response where numerous damage sensors and repair enzymes are recruited to the site of the break or mutation. Single stranded breaks (SSBs) are transiently detected by a multifaceted enzyme known as Poly-(ADP) ribosyl polymerase (PARP) which is recruited to the site of the break and undergoes rapid poly(ADP) ribosylation. This recruits other repair enzymes to the damaged site and initiates repair, while PARP dissociates to detect the next SSB. Double stranded breaks (DSBs) are detected by HR and NHEJ repair pathways which trigger ATM activation and recruitment of various repair enzymes. In NHEJ, the Ku70/80 heterodimer is the first to recognize and bind the DSBs within seconds and prevents improper end resection and serves as a scaffold to recruit other NHEJ components [40, 41]. 
Other components include DNA-PKcs, XLF, and ligases to rejoin the newly formed ends. In HR, an MRN complex composed of Meiotic Recombination 11 homolog (Mre11), DNA repair protein RAD50 (RAD50) and p95/Nibrin (NBS1) bind to the site of the break and recruit other factors such as Ataxia telangiectasia mutated (ATM), ataxia telangiectasia and Rad3-related protein (ATR), Breast cancer type 1 susceptibility protein (BRCA1), Tumor protein (P53) and others. Once ends are resected, the cell cycle and transcription may resume. Therefore, although DNA is constantly under threat of damage, robust repair pathways help to remove these lesions to allow survival and replication.

\section{Inflammation}

Obesity is a chronic disease where a significant increase in body mass results in excessive fat accumulation in and around various tissues as a result of excessive food consumption. Adipose tissue is divided into two types; the brown adipose tissue which possesses adipocytes with high concentrations of mitochondria that express uncoupling protein (UCP-1) responsible for the thermogenic activity of the tissue [7, 18, 42, 43], and the white adipose tissue, responsible for fat storage. However, recent studies have shown the white adipose tissue to also be an important endocrine organ responsible for hormonal regulation of various processes $[42,44,45]$. Due to the hyperplasia and hypertrophy that accompany obesity, adipocytes exhibit a lower density of insulin receptors and higher beta3 adrenergic receptor levels, which facilitates the diapedesis of monocytes, initiating a proinflammatory response [46]. WAT consists of different types of immune cells such as fibroblasts, pre-adipocytes, adipocytes and macrophages which have been shown to be significantly elevated in various metabolic liver diseases including diabetes, atherosclerosis and hypertension [47, 48]. Additionally, adipokines and adipocytokines such as plasminogen activator inhibitor-1 (PAI-1), tumor necrosis factor-alpha (TNF- $\alpha$ ), resistin, leptin, and adiponectin have also been measured [46, 47, 49]. These factors play an important role in the homeostasis of various physiological processes including regulating insulin sensitivity, fatty acid oxidation, lipolysis, and regulating appetite.

It has long been known that oxidative stress in involved in the pathological process of obesity, diabetes, cardiovascular disease and atherogenesis [49-51]. Although obesity may 
induce oxidative stress, oxidative stress in turn, is associated with increased production of inflammatory agents that contribute to the development of the metabolic syndrome [52]. Key biomarkers of oxidative damage are higher in obese individuals and directly correlate with increased BMI and percentage of body fat, LDL oxidation and triglyceride levels. Inflammation is characterized by vasodilation, vascular permeability and an increase in inflammatory cells such as neutrophils and cytokines such as TNF- $\alpha$, interleukin-1 (IL-1), IL-6 and various other chemokines [49]. These cytokines are in turn potent stimulators of ROS by macrophages and monocytes, and therefore a rise in cytokine concentrations could be another mechanism for increased oxidative stress. Additionally, when obesity persists for a long period of time, antioxidant sources can be depleted, decreasing the activity of enzymes such as SOD, CAT, GPx, TRX and PRDX. In fact, SOD and GPx activity have been shown to be significantly lower in obese individuals compared with healthy persons. further increasing the risk of tissue morbidity [53].

Interestingly, hibernation has been shown to confer resistance to inflammation-related injury where, despite the large increase in fat stores, the levels of pro-inflammatory agents seem to remain low over the winter dormancy [54]. It has been suggested that there could be an overall suppression of immune activation during the hibernating season $[18,54,55]$, however, we believe that inflammation is in fact being maintained at basal levels, in a tissue-specific and likely pathway-specific manner. NF- $\mathrm{kB}$, an important protein complex that controls cytokine expression and cell survival, was found to be activated in ground squirrel intestinal mucosa during hibernation compared with summer active squirrels [56]. However, studies in our lab on the same animal models found no significant increase in this transcription factor in any other tissue (unpublished results). This suggests that there could be another factor responsible for regulating the expression of inflammatory targets during the torpor-arousal cycle in the hibernating ground squirrel.

\section{Humanin}

The origin of mitochondria has been accepted to be due to endosymbiosis, where proteobacteria were engulfed by eukaryotic hosts and then integrated into their own systems [57]. This incorporation resulted in an evolutionary shift enabling a monumental upgrade 
where oxygen was used to mass-produce energy and other biosynthetic precursors. Owing to their origin, mitochondria are unique organelles in that they contain their own genome. This small genome in vertebrates typically encodes 13 protein-coding genes (coding for subunits of electron transport chain and oxidative phosphorylation proteins) a two ribosomal RNAs (12S \& 16S rRNAs), and 22 transfer RNAs (tRNAs). However, recent evidence shows that there are other genes hidden in the mitogenome. Mitochondria have diverse cellular functions including energy production, regulation of apoptosis, biosynthesis of precursors, heme synthesis, Fe-S cluster production, ion homeostasis and ROS production [58]. Despite the fact that they have such crucial responsibilities, little was known about how they transmitted information to the cytoplasm, until recently.

The discovery of a mitochondrially-produced small peptide both increased the known gene products encoded by mitochondria and provided the first example of peptidebased retrograde signaling (i.e. mitochondrial feedback to affect cytosolic processes). Humanin is a mitochondrial peptide that is highly conserved across species and was originally discovered in 2001 in the healthy portion of the brain from an Alzheimer's disease $(A D)$ patient $[59,60]$. Functional screening of clones protecting neuronal cells from amyloid precursor protein (APP)-related death identified multiple clones whose sequences were identical to a part of the mitochondrial 16S rRNA. Further functional analysis and sequence screening revealed a $75 \mathrm{bp}$ open reading frame sequence that yielded a 24 amino acid peptide, designated humanin. Translation of the peptide can be done in either the mitochondria or cytoplasm depending on the location of the translation machinery. Due to a slight difference in the genetic code between cytoplasmic and mitochondrial compartments, the resulting peptide is either a truncated 21 amino acid peptide when translated in the mitochondrion, or a 24 amino acid peptide if translated in the cytoplasm [61].

Humanin was shown to have several neuroprotective properties including antiapoptotic activity, as well as the ability to antagonize neurotoxicity from AD genes. Additionally, several cytoprotective effects have been described including protection against oxidative stress, atherosclerotic plaque formation, stimulating insulin sensitivity and suppressing apoptosis. Humanin mediates this protection through different pathways 
by binding to its specific receptor on target proteins. It interferes with the pro-apoptotic factor Bax, with insulin-like growth factor (IGF)-binding protein 3 (IGFBP3) and inhibits the signal transducer and activator of transcription 3 (STAT3) transcription factor (Szereszewski and Storey, manuscript; [62, 63]). Because humanin has been quite successful in the treatment against amyloid-beta-related stress, several labs have tested its ability to protect against oxidative stress. Treatment of rat retinal cultures have shown a protective phenotype from oxidative stress induced by hydrogen peroxide [64]. In human carotid atherosclerotic plaques, humanin was found to be twice as high in symptomatic patients compared with asymptomatic patients [65]. Humanin has been discovered in many other animal models including chimps, rats, bats, dogs, cows as well as ground squirrels (Szereszewski and Storey, manuscript). Knowing that the hibernating ground squirrel exhibits exceptional neuroprotection during hibernation through the synaptic plasticity of its neural structures, it is possible that the squirrel-specific peptide (s-humanin) plays a critical role in neuroprotection during the torpor-arousal cycle.

\section{Objectives and Hypotheses}

Since mammalian hibernators are subject to significantly increased levels of oxidative stress, especially upon exiting torpor, I chose for my Ph.D. thesis to explore one of the main protective mechanisms against oxidative damage as well as different damageassociated processes that enable the squirrel to survive the endogenous stress associated with arousal. I chose to approach this task at multiple levels including analyzing the regulation of the antioxidant response with a particular focus on peroxiredoxins and their function in mitigating cumulative oxidative stress (Chapter 2), the effects of increasing levels of oxidative stress and suppressed metabolic rate on the DNA-damage repair pathway (Chapter 3). The inflammatory response through the regulation of the IRF3 pathway and its induction of interferon-related genes (Chapter 4), and finally, the regulation and role of a cytoprotective mitochondrially-derived peptide, s-humanin (Chapter 5). Each of these themes was assessed over the time-course of the torpor-arousal bout and involved analysis of responses by diverse tissues (brain, liver, skeletal muscle, BAT and WAT) of the hibernating thirteen-lined ground squirrel (Ictidomys tridecemlineatus). The brain was 
selected for its importance in neuroprotection and plasticity during torpor, the liver was chosen due to its well-established role in metabolism and maintenance of homeostasis during hibernation, skeletal muscle was chosen due to its importance in shivering thermogenesis during arousal, and the adipose tissues were selected for their ability to rewarm the body with non-shivering thermogenesis (BAT), versus their endocrine function and role in providing lipids fuels (WAT). While each chapter is aimed at deepening the understanding of the effects of oxidative stress on each of these processes throughout the course of hibernation, this work also utilizes novel approaches and techniques to make significant advances in the field of hibernation and potentially provide novel avenues for the study of tissue wasting, regeneration and transplantation.

\section{Objective 1: Antioxidant enzymes}

As previously mentioned, in order to achieve a metabolic rate drop to $<5 \%$ of euthermic levels during hibernation, virtually all but the most important metabolic processes are suppressed. During this time, oxygen perfusion of tissues is less than $10 \%$ of euthermic rates and heart rate is slowed. However, upon arousal, the squirrel begins to quick breathe and oxygen returns to the tissues due to increased circulation and rewarming. An influx of reactive oxygen species also floods the cell creating the potential for oxidative stress. Initial studies on the thirteen-lined ground squirrel have confirmed that the arousal period is burdened with significantly elevated levels of toxic radicals which must be rapidly cleared to avoid damage to macromolecules. One important mechanism to mitigate this damage is the upregulation of the antioxidant defense. Key antioxidant enzymes including SOD, CAT, TRX and PRDX play a significant role in reducing toxic oxidants and protect the cell from damage. Previous work has shown PRDXs to be involved in the mitigation of damage from $\mathrm{H}_{2} \mathrm{O}_{2}$ as a ROS as well as controlling its action as a signaling molecule. Initial studies on the ground squirrel revealed PRDX1-3 to play a critical role during hibernation [7]. In this thesis, I greatly expand upon the analysis of antioxidant defenses and the regulation of other PRDX isoforms throughout the entirety of the torpor-arousal cycle to determine their expression during arousal, as a key time-point for oxidative stress. 


\section{Hypothesis 1 (Chapter 2):}

If antioxidant enzymes play a significant role in mitigating ROS production, then I hypothesize that PRDX isoforms will be generally enhanced during arousal compared to torpor.

Chapter 2 tests this hypothesis by exploring the overall antioxidant capacity of the tissues using an ELISA-based technique which measures the antioxidant system including enzymes such as SOD, CAT, GPx; macromolecules such as albumin, ceruloplasmin, ferritin; and small molecules including ascorbic acid, tocopherol, $\beta$-carotene, uric acid and bilirubin. The assay relies on the ability of antioxidants in a sample to inhibit the oxidation of ABTS (2,2'-azino-di-[3-ethylbenzthiazoline sulphonate]) to ABTS+ by metmyoglobin. The antioxidant capacity was measured in the liver and the brown adipose tissue across the torpor-arousal cycle. Furthermore, the relative protein expression levels of the classical antioxidant enzymes were measured in liver (comparable data for BAT and WAT was previously published). The abundance of SOD1, SOD2, CAT, TRX1 and PRDX2 were measured in control and late torpor using Luminex ${ }^{\circledR}$ multiplex analysis (Appendix 2.1). Chapter 2 then focused on peroxiredoxin expression in the liver, BAT and WAT since only PRDX (and TRX) levels appeared to be significantly increased in liver in response to torpor, whereas the other enzymes showed no significant change. Using western blotting, the relative protein expression levels of PRDX1-4, and 6 were measured throughout the torpor-arousal cycle in the thirteen-lined ground squirrel.

\section{Objective 2: DNA damage and oxidative stress}

Maintaining euthermia is energetically very costly and without proper fuel supplements, the rate of ATP expenditure will quickly overwhelm ATP production. As a result, winter hibernation includes strong metabolic rate depression and entry into torpor. This involves the targeted suppression of many metabolic processes while still ensuring that key pro-survival pathways remain active throughout bouts of torpor and arousal. However, this could potentially leave the ground squirrel susceptible to damage from 
oxidative stress if pro-survival or damage-repair pathways are suppressed or inadequate. Oxidative stress is potentially damaging to all macromolecules including DNA. Various modifications or mutations can occur in response to an oxidative environment such as single pyrimidine and purine base lesions, intrastrand cross-links, purine 5, 8cyclonucleosides, DNA-protein adducts, and intrastrand cross-links formed by the reactions of either the nucleobases or the 2-deoxyribose moiety [66-68]. In an attempt to protect DNA from damage, epigenetic markers may hold it in a condensed form to reduce transcription, however lesions may still occur. Especially during arousal, when there is a significant enhancement of oxidants, DNA is left vulnerable to attack. It has been demonstrated however, that the ground squirrel exits torpor without any significant damage to its tissues or genetic material. It was hypothesized that this was as a result of increased antioxidant defenses but also a lack of damage. However, I suggested that there is damage accumulating, but that repair pathways remain active to mitigate it. Hence, this chapter examined the differential regulation of the DNA damage repair pathway along with an important marker of damage across the course of the torpor-arousal cycle.

\section{Hypothesis 2 (Chapter 3):}

If DNA is getting damaged during the torpor-arousal cycle, then the DNA-damage response will be upregulated to protect it from and repair damage due to oxidative stress.

Chapter 3 tests this hypothesis by examining the relative expression changes of key DNA damage repair proteins in the liver and muscle: Poly-(ADP)-ribosyl polymerase (PARP), XLF, Ku80, the MRN complex (Mre11, Rad50, Nibrin), ATM, ATR and the phosphorylated forms of Nibrin (Ser343) and Mre11 (Ser676) to provide an overview of the DNA damage repair pathway. Additionally, the cytonuclear distribution of the PARP1 enzyme was assessed using western blotting to measure its translocation into the nucleus. Furthermore, an important marker of DNA damage, 8-hydroxy-deoxyguanosine, was measured using an ELISA to determine changes in relative levels between liver and skeletal muscle tissues during late torpor versus early arousal. 


\section{Objective 3: Interferon regulated genes}

Transcription factors play a vital role during hibernation. Despite the overall decrease in transcription and translation rates, various pathways must be selectively enhanced and transcription factors are vital to this response. Antioxidant enzymes and apoptotic factors are particularly important for maintaining cellular integrity and have been shown to be selectively maintained throughout the hibernation period. By contrast, the immune response has been thought to be generally suppressed as a means of conserving energy. Inflammatory targets that have been shown to be significantly upregulated in obese patients and in various metabolic diseases, do not appear to be elevated in the hibernating ground squirrel, despite a similar hyperphagic period.

A suppression of the inflammatory response during hibernation is unlikely to be able to signal the presence of foreign pathogens and therefore leaves the squirrel vulnerable during a period of metabolic rate suppression. Therefore, I postulated that select pathways are maintained or induced during pivotal time points such as arousal and that key transcription factors, such as the interferon-regulatory factors (IRFs) play an important role in controlling the expression of downstream interferon-related genes that will signal the presence of foreign invaders.

\section{Hypothesis 3 (Chapter 4):}

If damaging agents and pathogens are accumulating during the hibernation period, the IRF3 transcription factor will be upregulated in a tissue-specific manner and will induce the expression of downstream IFN-related genes in a time-specific manner in hibernating ground squirrels.

Chapter 4 tests this hypothesis by performing western blotting to assess IRF3 protein expression levels, in both total and phosphorylated forms, as well as its upstream regulators: TBK1, IKKe, MAVS, STING, RIG-I, and MDA5. Relative transcript levels of the IRF3 transcription factor were measured with quantitative PCR as well as the 
expression of downstream IFN-regulated genes such as cytokines, chemokines, and adipose-specific inflammatory targets in the liver, BAT and WAT of the hibernating ground squirrel throughout the torpor-arousal cycle.

\section{Objective 4: S-humanin}

A novel mitochondrially-derived peptide discovered in 2001 in a portion of the open reading frame of the $16 \mathrm{~S}$ rRNA of the mitochondrial genome was found to have neuroprotective properties and protect against Alzheimer's disease. This peptide has since been characterized in various other mammals and has been found to have many other cytoprotective properties including protection against oxidative stress in several tissues aside from the brain. This is of particular interest because the hibernating ground squirrel already exhibits remarkable neuroplasticity and cytoprotection of its tissues and therefore the squirrel-specific analogue of humanin could play a significant role in conferring stresstolerance during hibernation.

\section{Hypothesis 4 (Chapter 5):}

S-humanin (squirrel-specific humanin) will be upregulated in the tissues of the hibernating ground squirrel and will play a role in the cytoprotection of those tissues.

Chapter 5 tests this hypothesis by performing ELISA assays that measured the relative expression levels of s-humanin peptide across the torpor-arousal cycle, as well as PCR to examine the relative expression of s-humanin mRNA transcript levels. Computational analysis of the proposed 3-dimensional structure of s-humanin was also done and compared to the known molecular structure of humanin. Sequence conservation was analyzed between the human, squirrel and several other mammalian isoforms to determine patterns in sequence functionality. This provides an overview of the regulatory mechanisms of s-humanin during torpor and its role as an important cytoprotective peptide. 


\section{CHAPTER 2}

\section{DON'T (OXIDATIVE) STRESS - HOW HIBERNATING SQUIRRELS REGULATE PEROXIREDOXINS}




\begin{abstract}
Hibernation involves a period of profound metabolic rate depression that is employed by many mammals to avoid the harsh conditions of winter. However, transitions between euthermia and deep torpor have major consequences for body temperature, metabolic rate, and organ metabolism. In particular, during arousal from torpor, hibernator tissues are exposed to a rapid increase in oxygen uptake and consumption that, in turn, generates oxygen free radicals that are capable of damaging macromolecules. The present work aimed to investigate the contribution of the antioxidant response to damage control and conferring stress tolerance over the torpor-arousal cycle of 13-lined ground squirrels (Ictidomys tridecemlineatus). Using a combination of techniques including multiplex protein analysis, ELISA assays and western immunoblotting, the role of peroxiredoxins and their isoforms were analyzed in three key tissues: liver, brown adipose tissue (BAT), and white adipose tissue (WAT). An overall view of antioxidant capacity revealed tissuespecific regulation. Liver showed no significant changes across the torpor-arousal cycle, whereas BAT revealed a suppression of antioxidant defenses during the entrance stage into torpor (to just $69 \pm 8 \%$ of control values), as well as during interbout arousal (67 $\pm 4 \%$ of control), while remaining at baseline levels during late torpor and early arousal. Analysis of relative protein levels, showed that superoxide dismutase (SOD) and catalase did not appear to be induced during torpor in liver, whereas thioredoxin and peroxiredoxin protein expression increased significantly by 1.90 -fold and 2.25 -fold, respectively, compared with controls. A deeper focus into peroxiredoxins revealed significant increases in PRDX2, 3, 4 and 6 at selected time points in liver, PRDX2 and 3 in BAT, but only PRDX6 in WAT. Interestingly, selective suppression of various PRDX isoforms occurred at several time points. These data help to elucidate the molecular mechanisms of antioxidant enzyme regulation responsible for the stress tolerance of hibernating squirrels.
\end{abstract}




\subsection{Introduction}

Hibernation is a complex, yet fascinating, phenomenon that many mammals use to cope with the extreme challenges of winter. When temperatures fall below freezing, and ice covers the ground, it becomes increasingly difficult to forage for food. Many small mammals turn to an adaptive strategy known as hibernation, in which they depress their metabolic requirements until conditions again become more favorable. Hibernation is a cyclic process characterized by prolonged periods of torpor interspersed with brief periods of arousal back to euthermia [69]. For example, in 13-lined ground squirrels (Ictidomys tridecemlineatus) torpor can last for $\sim 1-2$ weeks interspersed with $<24 \mathrm{~h}$ periods of arousal. The metabolic depression of torpor is facilitated by many physiological changes including a strong decrease in body temperature to near-ambient levels (often $\sim 5^{\circ} \mathrm{C}$ ), a decrease in breathing rate (from 100-200 bpm to 4-6 bpm), a decrease in heart rate (from 200-300 bpm to 3-5 bpm), a switch from carbohydrate-based metabolism to lipid-based metabolism, and a decrease in organ perfusion rate $(<10 \%$ of euthermic values). Most significantly, hibernators decrease their metabolic rates and suspend non-essential energy-expensive cellular processes (e.g. transcription, protein turnover, ATP-pumps, etc.) [18, 21, 69], while promoting pro-survival functions (antioxidant response, heat shock protein response, antiapoptotic response, etc. [70-72]. In this way, small mammalian hibernators are able to conserve energy and rely mainly on their internal fuels for survival for the duration of hibernation. Due to the impressive drop in body temperature and metabolic rates, 13-lined ground squirrels are thus capable of achieving a balance between ATP supply and demand. To prepare for this metabolic reprioritization, the squirrel accumulates large internal lipid deposits in the white adipose tissue (WAT) during a period of hyperphagia in the summer, which are then used as the fuel source for $\beta$-oxidation during torpor $[13,18]$. In addition to fueling metabolism during torpor, fatty acid oxidation also fuels non-shivering thermogenesis in the mitochondria of the brown adipose tissue (BAT) during arousal to increase the animal's body temperature. These and other metabolic processes are under tight control by various mechanisms that modulate protein expression of select targets in multiple pathways. 
The most remarkable characteristic of hibernation is that squirrels undergo enormous physiological, molecular and biochemical changes during torpor, and exit from it without any significant damage to their tissues or cell components $[54,73]$. Aside from hibernation, arousal from hibernation and tissue reoxygenation also imposes major challenges that if not dealt with appropriately, can result in death. During this time, the rapid and large increase in respiration and metabolic heat production needed to rewarm the body leads to a comparable increase in reactive oxygen species (ROS) generation $[74,75]$. To cope with this major increase in ROS production, many cytoprotective pathways are induced and these pathways are a characteristic of metabolic rate suppression. Examples of these include upregulation of the heat-shock protein response, which allows folding of new proteins, repair misfolded proteins or prevents protein aggregation during these times of stress [18, $22,42]$, the induction of anti-apoptotic responses which aid cell preservation, and a strong increase in antioxidant defenses. Indeed, up-regulation of antioxidant enzymes is known to be a feature of many species that use metabolic rate suppression to endure prolonged periods of environmental stress [76-79].

Overproduction of ROS is capable of damaging multiple kinds of cellular macromolecules including DNA, proteins, lipid membranes, etc. However, maintenance of cellular homeostasis is generally achieved through the release of antioxidant defenses, which mitigate the damage caused by ROSs [7, 44]. The antioxidant response involves a range of enzymatic and non-enzymatic components. Among enzymes, superoxide dismutases (SODs), catalase and glutathione peroxidase act as the first line of defense against oxidative stress $[24,80]$. The mitochondrial SOD2 isoform neutralizes superoxide anion radicals $\left(\mathrm{O}_{2}^{-}\right)$produced by cellular respiration or $\beta$-oxidation [81, 82], and the same is achieved in the cytosol by SOD1. Hydrogen peroxide is also highly reactive and is reduced to water by catalase and glutathione peroxidase [24]. Enzymes and molecules involved in the second line of antioxidant defense include thioredoxin (TRX), thioredoxin reductase, and peroxiredoxin (a Cys-based peroxidase) [30] as well as members of the glutathione system (glutathione, glutathione reductasem glutathione peroxidase (GPx), and glutathione-S-transferases. 
These antioxidant enzymes have been well studied in numerous disease states $[50,83$, 84] as well as in our lab in response to diverse forms of environmental stress. Many have been found to be upregulated during the stress response, or during arousal, in a tissuespecific manner, and contribute to the "fingerprint" of metabolic rate suppression. Classical examples include SOD, GPx and ascorbate which all showed increased activity in BAT of hibernating ground squirrels, Citellus citellus. GPx was also elevated in liver, as was ascorbate in blood plasma [85]. In another mammalian hibernator, TRX1 activity was elevated over 2-fold in WAT, SOD activity rose by 1.1-fold in skeletal muscle as well as an increase in the antioxidant capacity in the ileum during torpor in the gray mouse lemur [86, 87]. Antioxidant enzymes in other stress-tolerant animals revealed similar trends; GPx increased in activity during whole body freezing in wood frogs (Rana sylvatica) [88] and catalase increased over 30 hours of anoxia in the skeletal muscle and heart. GPx activity also increases in the heart and brain of leopard frogs in response to anoxia [89]. Furthermore, catalase and GPx activity increased in the brains of hypoxic carp and SOD and catalase activity is increased during aerobic recovery in the kidney [78]. Many other animals show similar antioxidant defense patterns in response to the respective environmental stresses that they endure including land snails, African clawed frogs, goldfish, toads, goldenrod gall insects $[76,77,90,91]$ and more, thus supporting the molecular signature (or "fingerprint") of the stress-response program.

Many studies have focused on the first three antioxidant enzymes, but not as much is known about the regulation of peroxiredoxin (PRDX). PRDXs are a family of peroxidases with molecular weights between $20-30 \mathrm{kDa}$. They are present in organisms from all kingdoms, and exist in six isoforms in all eukaryotic cells [30]. PRDXs play a key role in reducing oxidative species, but also have diverse functions in the cell. Some of these include intracellular signaling of physiological responses such as proliferation, differentiation and migration [92-94], chaperone function [95], circadian rhythm regulation [96], and cellular immunity where PRDXs act as pathogen-associated molecular patterns (PAMPs) to elicit an immune response by secreting hydrogen peroxide from phagosomes to kill microorganisms [97]. Rising levels of toxic oxidants can be obviously destructive to the cell but certain oxidants, such as $\mathrm{H}_{2} \mathrm{O}_{2}$, have been described as a "necessary evil" for the cell. 
Antioxidant defense systems play an important role in mitigating damage caused by ROS and other oxidants such as $\mathrm{H}_{2} \mathrm{O}_{2}$. One of the challenges in the field is the elucidation of the mechanisms by which $\mathrm{H}_{2} \mathrm{O}_{2}$ is sensed and how the signal is propagated in cells. Redox regulated proteins play key roles in many signaling pathways that are at the basis of various pathologies, including obesity, cardiovascular diseases, diabetes and many others [98]. Furthermore, peroxiredoxins have been associated with various cancers [99], neurodegenerative [100], cardiovascular [101] and metabolic diseases [51]. To identify the molecular patterns of antioxidant defenses, the present study assessed the regulation of antioxidant defenses with a particular focus on PRDXs as key players in $\mathrm{H}_{2} \mathrm{O}_{2}$ destruction in during hibernation. Global antioxidant capacity was measured in a group of selected tissues, relative expression levels of 5 antioxidant enzymes was assessed via multiplex analysis, and the relative protein expression of PRDX isoforms was determined over the course of the torpor-arousal cycle in liver and BAT of hibernating ground. Identifying the mechanisms of $\mathrm{H}_{2} \mathrm{O}_{2}$ signal transduction will increase our understanding of this stressresponse and will potentially provide the basis for therapeutic interventions in redox-related diseases.

\subsection{Materials and Methods}

\section{Animal experiments}

Animal experiments were conducted as previously described [102]. All animal experiments were conducted by in the laboratory of Dr. J.M. Hallenbeck lab (NINDS, Bethesda, Maryland) and approved by the Institutional Animal Care and Use Committee. Equal numbers of male and female squirrels ( 5-10) approximately between 1 and 3 years of age were sampled for this experiment and an $n=4$ squirrels were used for each experimental condition. Following capture, squirrels were individually housed in shoebox cages in a cold room with an ambient temperature of $21^{\circ} \mathrm{C}$ under a $12 \mathrm{hr}$ light/12 hr dark cycle. All animals were intrapetitoneally implanted with sterile radio-telemetric transmitters (IPTT-300, Bio medic Data Systems) to measure body temperature during the torpor-arousal cycle. Once removed from the hibernation chambers, animals were briefly anesthetized with 5\% isofluorane and sacrificed by decapitation within 2 minutes. Tissue samples were retrieved from the following conditions: EC designates euthermic in the cold 
room; these squirrels had a stable $\mathrm{Tb}\left(\sim 37^{\circ} \mathrm{C}\right)$ and high metabolic rate for at least three days. EC squirrels were capable of entering torpor but had not entered torpor in the past 72 hours. $\mathrm{EN}$ designates entrance; animals in the entrance phase of the torpor bout with decreased $\mathrm{Tb}$ $\left(\sim 25-18^{\circ} \mathrm{C}\right)$. LT designates late torpor; animals that remained in deep torpor for 5 days and have not begun a periodic arousal ( $\left.\mathrm{Tb} \sim 5-8^{\circ} \mathrm{C}\right)$. EA designates early arousal; characterized by an increased respiratory rate of more than 60 breaths per minute and a rising body temperature around $9-12^{\circ} \mathrm{C}$. Finally, IA designates interbout arousal; animals that were naturally aroused after the torpor bout of the hibernation cycle and reached the respiratory rate, metabolic rate and body temperature of fully aroused animals for up to 18 hours after being in torpor for up to 5 days. Samples of liver, brown adipose tissue (BAT), and white adipose tissue (WAT) were collected and were flash-frozen in liquid nitrogen, shipped on dry ice to Carleton University, and then stored in $-80^{\circ} \mathrm{C}$ until use. The highest standards in ethics and transparency that are applied in North America were used for all experiments. This study, including animal housing and experimental protocols, were approved by the NINDS institutional animal care and use committee (IACUC; Permit Number ASP 1223$05)$.

\section{Total soluble protein extracts}

Protein extracts were prepared from frozen tissue samples according to the Millipore assay kit instructions. Approximately $50 \mathrm{mg}$ of frozen tissue samples from different time-points of the torpor-arousal cycle ( $\mathrm{n}=4$ independent biological replicates) were immediately homogenized in 1:4 w/v lysis buffer (EMD Millipore Cat \#43-045) containing $1 \mathrm{mM} \mathrm{Na}_{3} \mathrm{VO}_{4}, 10 \mathrm{mM} \beta$-glycerophosphate, $10 \mathrm{mM} \mathrm{NaF}$, and $10 \mu \mathrm{L}$ of protease inhibitor cocktail (Bioshop Cat \#PIC001) for every $1 \mathrm{~mL}$ of lysis buffer using a Dounce homogenizer. After incubation on ice for 30 min with intermittent vortexing, samples were centrifuged at $12,000 \times \mathrm{g}$ for $20 \mathrm{~min}$ at $4^{\circ} \mathrm{C}$, and the supernatants were collected and stored at $-80^{\circ} \mathrm{C}$ until further use. For multiplex analysis, the protein concentrations of each sample was determined using the Bio-Rad assay (Cat \#500-0005) immediately before the assay, and the concentrations were standardized to $5 \mu \mathrm{g} / \mu \mathrm{L}$ using lysis buffer. Aliquots of each sample were diluted in Milliplex MAP assay buffer (Cat\# 43-010) in at least a 1:1 ratio per tissue to reach a working concentration of $25 \mu \mathrm{g} / \mathrm{well}$. 


\section{Luminex assays}

The antioxidant enzyme Luminex ${ }^{\circledR}$ panel (Cat\# HOXSTMAG-18K) was purchased from EMD Millipore and was used according to the manufacturer's instructions. The 5plex Oxidative stress Magnetic Bead Panel was used to measure the effects of 5 antioxidant enzymes: catalase, SOD1, SOD2, thioredoxin (TRX1) and peroxiredoxin (PRDX2) in liver of the thirteen-lined ground squirrel Ictidomys tridecemlineatus during control and torpor. Assay buffer was used as a negative control, and unstimulated HepG2 Cell Lysate (Cat \#47-231) was used as a positive control according to the manufacturer's instructions. The cell lysate was provided as a lyophilized stock, which was reconstituted in $100 \mu \mathrm{L}$ of ultrapure water, yielding a final concentration of $2 \mathrm{mg} / \mathrm{mL}$. The squirrel samples were first tested on a standard curve to determine the best working concentration and were then combined with the appropriate amount of assay buffer prior to use to yield a final concentration of 15 or $20 \mu \mathrm{g} / \mathrm{well}$ for liver and BAT respectively. Antibody-immobilized beads (1X) (Cat \#HOSTPMX5-MAG) were prepared by sonicating, vortexing and diluting as instructed by the manufacturer. Equal volumes of the antibody-immobilized beads were added to each well, and the plate was incubated for 2 hours at room temperature (RT) on a plate shaker (600-800 RPM) while protected from light. Next, the beads were separated from excess assay mixture using a Magnetic Separator Block (Cat \#40-285), and the unbound proteins were decanted. Wells were washed three times with $200 \mu \mathrm{L}$ of assay buffer. Following washing, biotin-labelled detection antibodies (Cat \#HOXST-1018) were added to each well, and the plate was incubated for $1 \mathrm{~h}$ at RT on a plate shaker protected from light. Excess biotin-labelled detection antibodies were removed and the plate was washed 3 times with assay buffer. Next, $50 \mu \mathrm{L}$ of Streptavidin-Phycoerythrin (SAPE) (Cat \#MC-SAPE6) was added to each well and the plate was incubated protected from light on a plate shaker for $30 \mathrm{~min}$ at RT. Finally, the well contents were removed as described previously, wells were washed with assay buffer, and $100 \mu \mathrm{L}$ of Sheath Fluid was added to each well and the plate was incubated while shaking for $5 \mathrm{~min}$ at RT before measurements were taken using a Luminex 100/200 Instrument with xPonent Software (Luminex Corporation). Quantification and statistical analysis were performed using the net median fluorescence intensity (MFI) of a population of measurements (minimum bead count of 50, 
sample size of $100 \mu \mathrm{L}$, gate settings of $8000-15,000)$, and subtracted from background wells in order to determine the relative protein levels.

\section{Total protein preparation for antioxidant capacity assay}

A commercially available antioxidant capacity kit was purchased from Cayman Chemical Company (Cat \#709001). Total protein lysates (described above) were used for this assay. Briefly, a standard curve was prepared by combining increasing amounts of Trolox (6-hydroxy-2,5,7,8-tetramethylchoman-2-carboxylic acid) diluted in antioxidant assay buffer ( $5 \mathrm{mM}$ potassium phosphate, $\mathrm{pH} 7.4$, with $0.9 \%$ sodium chloride and $0.1 \%$ glucose) to obtain concentrations between 0 and $0.330 \mathrm{mM}$. Then, $10 \mu \mathrm{L}$ of Trolox solution and $2.0-5.0 \mu \mathrm{g} / \mu \mathrm{L}$ of protein samples from each tissue were combined with $10 \mu \mathrm{L}$ of metmyoglobin and $150 \mu \mathrm{L}$ of chromogen. Reactions were initiated by the addition of 40 $\mu \mathrm{L}$ of the hydrogen peroxide working solution before the plate was incubated on a shaker away from light for $5 \mathrm{~min}$ at RT. Absorbances were read at 750 and $450 \mathrm{~nm}$ and quantified as Trolox equivalents ( $\mathrm{mM} / \mathrm{mg}$ wet mass) based on the Trolox standard curve and therefore represent the antioxidant strength of the sample, compared to units of Trolox. The relative fold change of each time compared to control was reported. Due to low protein concentration, white adipose tissue was not assessed for its antioxidant capacity.

\section{Total protein preparation for immunoblotting}

Total protein lysates (described above) were used for western immunoblotting. Briefly, protein concentrations were determined using Bio-Rad reagent dye (Bio-Rad Cat\# 500-0006) and were all normalized to $10 \mu \mathrm{g} / \mu \mathrm{L}$ in multiplex assay buffer. Aliquots were then mixed 1:1 (v/v) with 2X loading buffer (100 mM Tris base $\mathrm{pH} 6.8,4 \% \mathrm{w} / \mathrm{v}$ SDS, 20\% $\mathrm{v} / \mathrm{v}$ glycerol, $0.2 \% \mathrm{w} / \mathrm{v}$ bromophenol blue, and 10\% v/v 2-mercaptoethanol), to yield a

final protein concentration of $5 \mu \mathrm{g} / \mu \mathrm{L}$. Samples were boiled for 10 minutes and then stored at $-20^{\circ} \mathrm{C}$ until use. Depending on the target, 20-30 $\mu \mathrm{g}$ of protein was loaded into each well of SDS-PAGE gels and electrophoresed until the desired separation was reached. After transfer to a PVDF membrane (160 mA for $90 \mathrm{~min}$ ), membranes were blocked with $1-5 \%$ of skimmed milk in Tris-buffered saline with $0.05 \%$ Tween (TBST) for 30 mins and probed with each respective primary antibody (Abclonal: PRDX1 Cat\# A1842, PRDX2 
Cat\# A1919, PRDX3 Cat\# A3076, PRDX4 Cat \#A1486, PRDX6 Cat \#A2031) (diluted 1:1000 in TBST), overnight at $4{ }^{\circ} \mathrm{C}$ on a rocking platform. The next day, membranes were washed and probed with an anti-rabbit IgG HRP-linked secondary antibody (diluted 1:6000 in TBST) for $30 \mathrm{~min}$ at room temperature on a rocking platform.

\section{Quantification and Data Analysis}

For the antioxidant capacity assay, the mean absorbances for each time point and tissue were calculated $( \pm S E M)$ for $n=4$ independent tissue extracts from different animals. Control values were normalized to 1 and all other time points were compared relative to the euthermic control. For western blotting, bands densities were visualized using Luminol and hydrogen peroxide using enhanced chemiluminescence on a ChemiGenius Bio-Imager (Syngene, Frederick, MD) and quantified by densitometric analysis using GeneTools Software. Band intensity of each lane was standardized against the summed intensity of a group of Coomassie-stained bands in the same lane to correct for variation in sample loading [103]. Mean normalized band densities ( \pm SEM) were calculated for control and torpor samples and data were expressed from $n=4$ independent tissue extracts from different animals. All data were analyzed either using a Student's T-test (control, torpor) or a one-way ANOVA (time course data) to identify significant differences between the groups $(p<0.05)$. For each figure, values that are statistically significant from control have an asterisk $(*)$ or have different letter notation. All statistical analysis and figure generation was performed using RBioplot [104]. 


\subsection{Results}

Total antioxidant capacity of liver and BAT were assessed over the torpor-arousal cycle (Figure 2.1). Liver tissue showed no significant change in antioxidant capacity throughout the cycle, but BAT revealed some significant changes. During entrance into torpor (EN), antioxidant capacity decreased to $69 \pm 8 \%$ of euthermic control values (EC), followed by a return to control levels during late torpor (LT). This was followed by a slight decrease to $80 \pm 2 \%$ of control values during early arousal (EA) and then by a slightly larger decrease to $67 \pm 4 \%$ of EC values during interbout arousal (IA).

Multiplex analysis of key antioxidant enzymes revealed significant increases in two of the five key enzymes during torpor in liver. Thioredoxin 1 and peroxiredoxin 2 increased in protein levels during torpor relative to control conditions (Figure 2.2). Thioredoxin 1 was found to increase by $1.90 \pm 0.14$-fold during torpor and peroxiredoxin 2 (PRDX2) increased by $2.25 \pm 0.17$-fold during torpor relative to control values.

To further analyze the regulation patterns of the peroxiredoxin isoforms, protein expression levels of isoforms 1-4 and 6 were measured across the torpor-arousal cycle. In liver, PRDX1 was decrease significantly during late torpor to $65 \pm 0.7 \%$ compared to control values. Early arousal and interbout arousal were at $74 \pm 8 \%$ and $70 \pm 3 \%$ of control values, respectively; these were not significantly different from EC values but different compared with EN (Figure 2.3). PRDX2 levels were generally unchanged aside from a significant increase of $2.60 \pm 0.33$-fold during late torpor, which also corresponds to the multiplex data (Figure 2.2). PRDX3 expression was also generally unchanged aside from a decrease during early arousal to $67 \pm 3 \%$ of control values. PRDX4 increased in expression by $1.44 \pm 0.06$-fold during late torpor and remained elevated over the rest of the cycle at $1.45 \pm 0.02$ and $1.35 \pm 0.09$-fold in early arousal and interbout arousal, respectively. Finally, PRDX6 expression was significantly increased during entrance to $1.97 \pm 0.14$-fold compared to euthermic control, followed by a return to control values during late torpor. However, another significant increase to $2.01 \pm 0.13$-fold occurred during early arousal followed by a return to control values during interbout arousal. 
Next, PRDX expression was examined in brown adipose tissue and revealed differential regulation in comparison to the liver. In BAT, PRDX1 was significantly suppressed during entrance to $57 \pm 8 \%$ of control values followed by a return to control values across the rest of the cycle. PRDX2 was found to be unchanged between control and late torpor but exhibited a strong response during early arousal and interbout arousal whereby protein expression levels were increased to $1.73 \pm 0.07$-fold for both time points. PRDX3 levels increased significantly during entrance by $1.29 \pm 0.06$-fold, followed by a further increase to $1.57 \pm 0.04$-fold during late torpor compared to EC. Then, values returned to control levels during early arousal, but rose again to $1.50 \pm 0.03$ during interbout arousal. PRDX4 levels revealed a significant increase during entrance by $1.25 \pm 0.01$-fold over control values followed by a significant drop to $58 \pm 1 \%$ of EC values during late torpor. During arousal, expression levels returned to control values followed by a drop to $92 \pm 1 \%$ of EC but were only significantly reduced compared to arousal. Finally, PRDX6 levels were assessed and generally revealed no significant change aside from a decrease in expression during entrance to $65 \pm 5 \%$ of EC followed by a return to control levels during late torpor and the rest of the time-course.

Finally, protein expression levels were measured in white adipose tissue. Interestingly, the majority of targets were actually found to either have no significant change or decrease in expression, in contrast to BAT. PRDX1 showed a decrease in expression during late torpor and maintained this suppression throughout the rest of the cycle. A significant decrease to $27 \pm 7 \%, 23 \pm 4$, and $22 \pm 3 \%$ respectively was seen during late torpor, early arousal and interbout arousal, respectively. Compared to EC, PRDX2 showed change in expression over the time course except for a significant decrease during early arousal to $40 \pm 1 \%$ of control values followed by a return to control levels during interbout arousal. PRDX3 was immediately suppressed during entrance to $27 \pm 7 \%$ of EC values followed by a slight increase to $62 \pm 4 \%$ of control during late torpor. During early arousal, values returned to control levels, but were again suppressed during interbout arousal to $54 \pm 4 \%$ of EC. PRDX4 showed a similar trend whereby a significant decrease was seen during EN to $51 \pm 11 \%$ of control levels, followed by a return to control values during LT. However, a significant drop then occurred during early arousal and interbout 
arousal to $33 \pm 3 \%$ and $47 \pm 6 \%$ of EC. Finally, PRDX6 showed relatively little change aside from a significant increase to $1.53 \pm 0.02$-fold during early arousal.

\subsection{Discussion}

Enhancement of antioxidant responses is an important feature of hypometabolism that has a duel function: first, the protection of macromolecules from oxidative damage and second, the mitigation of rising ROS levels during arousal. Torpor is a time when an organism cannot afford to maintain the high energetic expenditures associated with repairing, degrading, resynthesizing or refolding macromolecules that have been damaged by ROS or free radicals, and arousal is a period when metabolic rate increases rapidly, resulting in an influx of toxic oxidants into the cell. Oxygen consumption is significantly increased during arousal by as much as 36-fold higher than in torpor [105]. Much of this oxygen consumption is funneled into uncoupled respiration in the brown adipose tissue to drive thermogenesis [18]. Incidentally, the brown adipose tissue has been shown to have increased antioxidant defenses during this time, likely as a mitochondrial protective mechanism [85]. It seems that the increase in antioxidant defenses in this tissue is designed to protect the BAT against oxidative damage when there is an enormous increase in the metabolic activity in this tissue during arousal due to its pivotal function in rewarming. This study aimed to deepen understanding of the antioxidant response and the involvement of peroxiredoxins during hibernation in thirteen-lined ground squirrels.

The overall antioxidant capacity of BAT and liver revealed different patterns across the torpor-arousal cycle. As previously mentioned, studies on various hibernating species have revealed similar patterns whereby the antioxidant response is elevated during critical time points of the cycle. The liver revealed a maintenance of overall antioxidant capacity at control levels throughout the entire torpor arousal cycle, however interestingly, the brown adipose tissue revealed a significant decrease in antioxidant capacity. Previous work in our lab has shown antioxidant capacity to be 1.56-fold higher in torpid squirrel skeletal muscle compared to summer active animals, suggesting a maintenance of acute muscle performance [106]. Studies on other animals appear to have varying results whereby total antioxidant capacity is generally unchanged during periods of deep torpor. Another study 
examining hatchling turtle brain and liver found no significant change in antioxidant capacity in response to supercooling $(48 \mathrm{~h})$, freezing $\left(-2.5^{\circ} \mathrm{C}\right)$, or hypoxia $\left(4^{\circ} \mathrm{C}\right)$ [107]. This could suggest that during the period of hypometabolism, antioxidant defenses are generally suppressed but could be induced during periods of arousal, or solely in a tissue-specific manner. Furthermore, antioxidant capacity measures the overall change in the antioxidant response, as a balance between the antioxidant activities of several enzymes, vitamins and other targets, against free radicals. Therefore, an increase in certain antioxidant enzymes could be balanced out by a decrease in others and would therefore mask a significant response. Therefore, in order to focus on the specificity of the antioxidant response, the relative changes of key enzymes were examined.

Several antioxidant enzymes play important roles in mitigating damage caused by ROS and decrease the levels of oxidants in the cell. Classical examples are SOD, catalase and glutathione peroxidase. In this study, the levels of SOD1 and 2 as well as catalase were unchanged during torpor. Previous work in our lab however, showed that levels of these enzymes were differentially expressed in the brown and white adipose tissues [42]. Although BAT showed no changes in the present study, WAT revealed a significant increase in both SOD isoforms during late torpor but all other time points remained at control levels suggesting a tissue-specific antioxidant response. Other studies revealed similar results where SOD activity showed tissue-specific results. In the ground squirrel, SOD activity was measured in liver, heart and brain of torpid squirrels but revealed no significant change, whereas catalase activity was higher in heart and brain of torpid squirrels [108]. Therefore, it would appear that there is a multi-layered regulation of antioxidant defenses depending on the tissue requirements during that time. Another possible explanation for the lack of oxidative stress and damage is that the ischemiareperfusion associated ROS production may simply be avoided in hibernators possibly by tolerating it without initiation of aberrant ROS production [108]. Although hypoxia does not occur in the hibernating squirrel, it is possible that the arousal period simply does not result in the same burst of mitochondrial ROS that occur in non-hibernators and therefore does not require an increase in all antioxidant enzymes. 
Less is known about peroxiredoxin expression, although these enzymes have important functions as both regulators of the antioxidant response and as signaling molecules. Peroxiredoxins are a ubiquitous family of antioxidant enzymes that exert their protective antioxidant role through their peroxidase activity, whereby hydrogen peroxide, peroxinitrite and a wide range of hydroperoxides are reduced and detoxified [109]. PRDXs are divided into three classes; typical 2-Cys Prxs (PRDX1-4), atypical 2-Cys Prx (PRDX5), and 1-Cys Prx (PRDX6). These enzymes all share the same basic catalytic mechanism, in which an active-site cysteine is oxidized to sulfenic acid by the peroxide substrate [109]. The present data revealed a significant increase in PRDX2 in liver of hibernating squirrels and an increase in TRX1, the reducing partner of PRDX [33]. A parallel study performed in our lab found unchanged levels of PRDX2 in BAT and WAT, but a significant increase was noted in TRX1 during torpor [42]. Therefore, it can be assumed that the increases seen in liver PRDX and TRX are as a result of an increased antioxidant response whereas the TRX in BAT and WAT serves a primarily a cytoprotective function, in response to ischemia-reperfusion. This reflects a potential strategy by which the squirrel can protect its tissues from elevated ROS during a period of rewarming and reperfusion that will shortly follow. Peroxiredoxins may therefore help to decipher a pattern of oxidative stress resistance capacity during hibernation.

To further investigate PRDX regulation, the expression levels of their isoforms was examined. In this study, the relative protein expression levels of PRDX 1-4 and 6 were examined in liver, BAT and WAT and revealed differential expression patterns. In the liver, PRDX2, 4 and 6 were upregulated at various points of the torpor-arousal cycle. PRDX1 was generally suppressed as well as PRDX3, but only during early arousal (Figure 2.3). In BAT, PRDX2 was elevated during the arousal stages, but PRDX3 also showed a significant increase during all time points other than early arousal (Figure 2.4). Finally, WAT generally showed suppression of many of the isoforms across the torpor-arousal cycle aside from a small increase in PRDX6 during early arousal (Figure 2.5).

It has been shown that increased levels of peroxiredoxins (such as PRDX2) may inhibit the activation of several pathways including the platelet-derived growth factor (PDGF) receptor [110]. Studies using PRDX2 knockout mice have reported the activation 
of JNK and p38 MAPK pathways [93, 111] and therefore, modification of these enzymes during hibernation could result in changes in signal transduction at different stages of the hibernation cycle. Thioredoxins are implicated in feedback mechanisms to reactivate tyrosine phosphatases, which de-activate MAPK [93]. Therefore, thioredoxin oxidation could increase the extent of MAPK activation. This is further supported by a study from our lab where MAPK and p38 MAPK levels decreased in the liver of hibernating squirrels but were elevated in other tissues, such as the skeletal muscle [112,113]. Aside from WAT, PRDX2 was upregulated during late torpor in liver, and during early arousal and arousal in BAT. This suggests that perhaps various signaling pathways are being preferentially selected during various stages of the torpor-arousal cycle, in a tissue-specific manner.

PRDX3 is generally localized in mitochondria where its expression is induced by the c-Myc target gene. PRDX3 is required for c-Myc-mediated proliferation, transformation and apoptosis as well as for maintaining normal mitochondrial function [114]. Therefore, it would make sense that PRDX3 is significantly upregulated in BAT which is highly dependent on mitochondrial function for thermogenesis. However, it is interesting that PRDX3 was not significantly upregulated during early arousal - a key time during which high levels of thermogenesis are occurring although perhaps PRDX2 is more protective in the BAT at this time. In fact, a significant increase in PRDX2 expression was seen in BAT during early arousal, and this result is further supported by other work done in our lab [7]. However, it is also plausible that PRDX3 expression is induced earlier in the cycle in preparation for the generation of toxic radicals, and then expression levels are maintained throughout the remainder of the cycle. The increase seen during interbout arousal could then be to remove any other oxidants newly produced or remaining. Another study examining PRDX3 levels in mice revealed decreased expression in PRDX3 in obese mice and human subjects. PRDX3 knockout mice had increased fat mass, increased adipogenic transcription factors and lipogenic gene expression compared to wild-type mice due to adipocyte hypertrophy [115]. This could suggest that the ground squirrel regulates PRDX3 expression to maintain adipose function until such a time that it is needed, such as during torpor for beta-oxidation. 
PRDX4 is another crucial antioxidant enzyme that has been reported to be significantly cytoprotective against the initiation and development of various metabolic syndromes. It has been reported to protect against diabetes mellitus, atherosclerosis, insulin resistance, nonalcoholic fatty liver disease, inflammatory cytokines and certain apoptotic activities [116]. PRDX4 has been shown to regulate apoptosis whereby PRDX suppression or knockdown increases apoptosis. One study examined the effects of PRDX4 suppression and noted an increase in apoptosis in glioblastoma [117]. Another study found that PRDX4 overexpression in IL-1 $\beta$-induced chondrocytes decreased apoptosis through PI3Kdependent signaling [118]. Incidentally, pro-apoptotic pathways were not activated during hibernation in the thirteen-lined ground squirrel as evidenced by the regulation of several anti-apoptotic factors such as Bcl-2 and Bcl-xL [119]. Therefore, it is possible that PRDX4 may play an important role in this regulation and offers some level of cytoprotection during hibernation. PRDX4 has also been shown to reduce the number of 8-OH-dG-positive cells in cholestatic liver injury (8-OH-dG being a marker of DNA damage) [116]. It is proposed that PRDX4 reduces extracellular oxidant stress and mitochondrial dysfunction by suppressing the release of inflammatory cytokines and neutrophil infiltration. Our study revealed the strongest PRDX4 response in liver, which is consistent with these findings. These results suggest that PRDX4 may be playing a significant cytoprotective role in various tissues, namely the liver and BAT during the course of hibernation.

Finally, the expression of PRDX6 revealed a maintenance or increase in protein expression in all three tissues, aside from entrance in BAT. PRDX6 is the only isoform classified as a 1-Cys PRDX and uses glutathione and ascorbate as electron donors [120]. PRDX6 not only functions as a potent antioxidant enzyme, but also stabilizes mitochondrial function during ischemia-reperfusion [120]. It is also a key mediator in hypoglycemia in type 2 diabetes glucose metabolism due to its implication in glucose-dependent insulin secretion [121] as well as providing a non-redundant role in myocardial ischemiareperfusion injury where CAT and GPx cannot satisfy [122]. PRDX6 is regulated by a variety of transcription factors. Its expression can be suppressed by NF-кB [28], or can be induced by tumor-necrosis factor (TNF- $\alpha)$ and nuclear factor erythroid 2-related factor 2 (Nrf2). Nrf2 has been shown to be an important regulator of the transcription of many antioxidant genes, including peroxiredoxins, through binding to the antioxidant response 
element [123]. Studies examining Nrf2 expression during squirrel hibernation have shown it to be differentially regulated in a tissue-specific manner. Transcript and protein expression levels of Nrf2 increased in the heart of torpid ground squirrels [124]. Additionally, Nrf2 protein levels were significantly increased in BAT and liver of hibernating squirrels, but were unchanged in WAT, lung, and muscle and were reduced in brain and kidney during torpor [125]. This suggests that Nrf2 could be inducing the expression of PRDX6 during torpor and at various other stages of the torpor-arousal cycle in an effort to balance the antioxidant response. PRDX6 could also be exerting a cytoprotective response to ischemia-reperfusion during the arousal stage in liver and WAT of the hibernating ground squirrel.

In summary, the present study provides an overview of the pattern of antioxidant regulation in the liver, BAT and WAT during ground squirrel hibernation. The overall antioxidant response is a highly regulated and specific and functions to not only protect cell components from damage, but also to mitigate the increased levels of reactive oxygen species in the cell. Although many enzymes control this response, the importance of peroxiredoxins is becoming increasingly clear. PRDX2, 3, 4, and 6 appear to play significant roles in this response at varying time points in the torpor-arousal cycle. In addition to balancing antioxidant capacity, acting as signaling molecules and cytoprotectants, peroxiredoxins also maintain mitochondrial integrity and function. Elucidating the mechanisms that promote increased antioxidant defenses during hibernation in the thirteen-lined ground squirrel will contribute significantly to our understanding of metabolic rate depression and could potentially lead to applications for limiting tissue damage in various medical or organ preservation applications. 


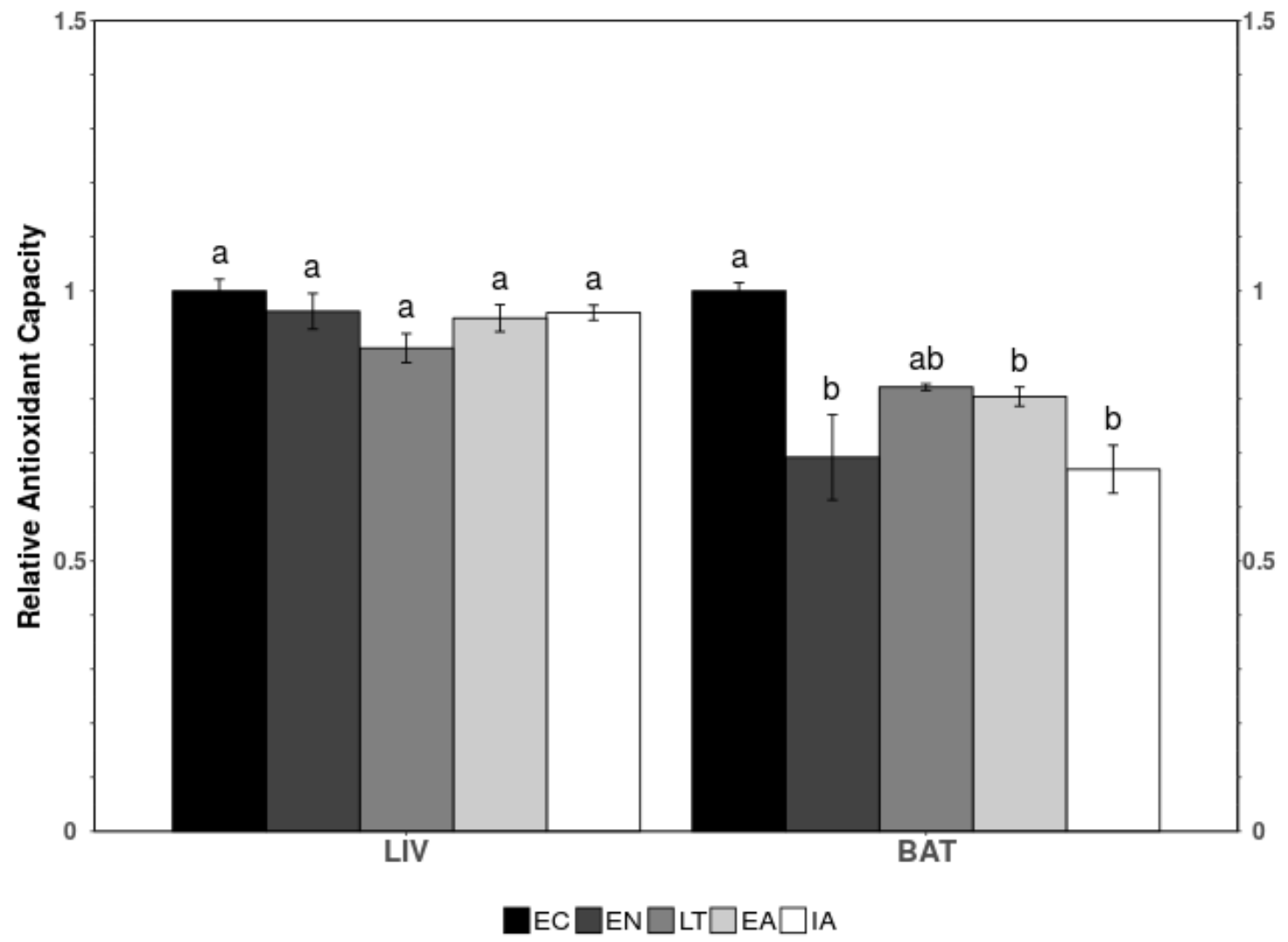

Figure 2.1: Relative antioxidant capacity in the liver and brown adipose tissue across the torpor-arousal cycle. Data are mean $\pm \operatorname{SEM}(n=4$ independent protein isolations from different animals). Data were analyzed using an analysis of variance followed by a Tukey post-hoc test $(p<0.05)$; values sharing the same letter notation are not statistically significant from each other. 


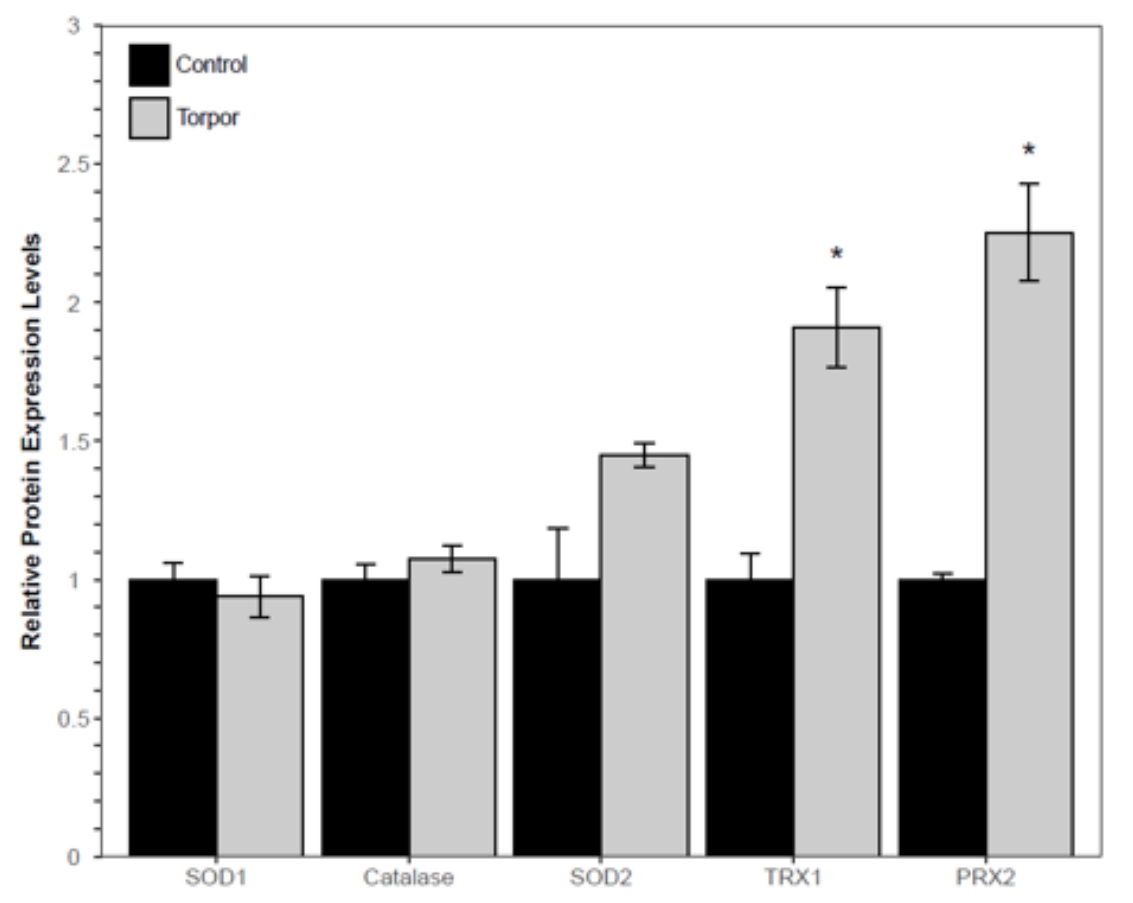

Figure 2.2: Relative protein expression of the antioxidant enzymes SOD1, CAT, SOD2, TRX1, and PRX2 in the liver of I. tridecemlineatus in control versus late torpor. Histogram representing the net mean fluorescent intensity as measured by Luminex ${ }^{\circledR}$. Data are mean $\pm \operatorname{SEM}(n=4$ independent protein isolations from different animals). Data were analyzed using the Student's T-test $(p<0.05)$; values with an asterisk $\left(^{*}\right)$ are statistically significant from the corresponding EC control. 
(A)

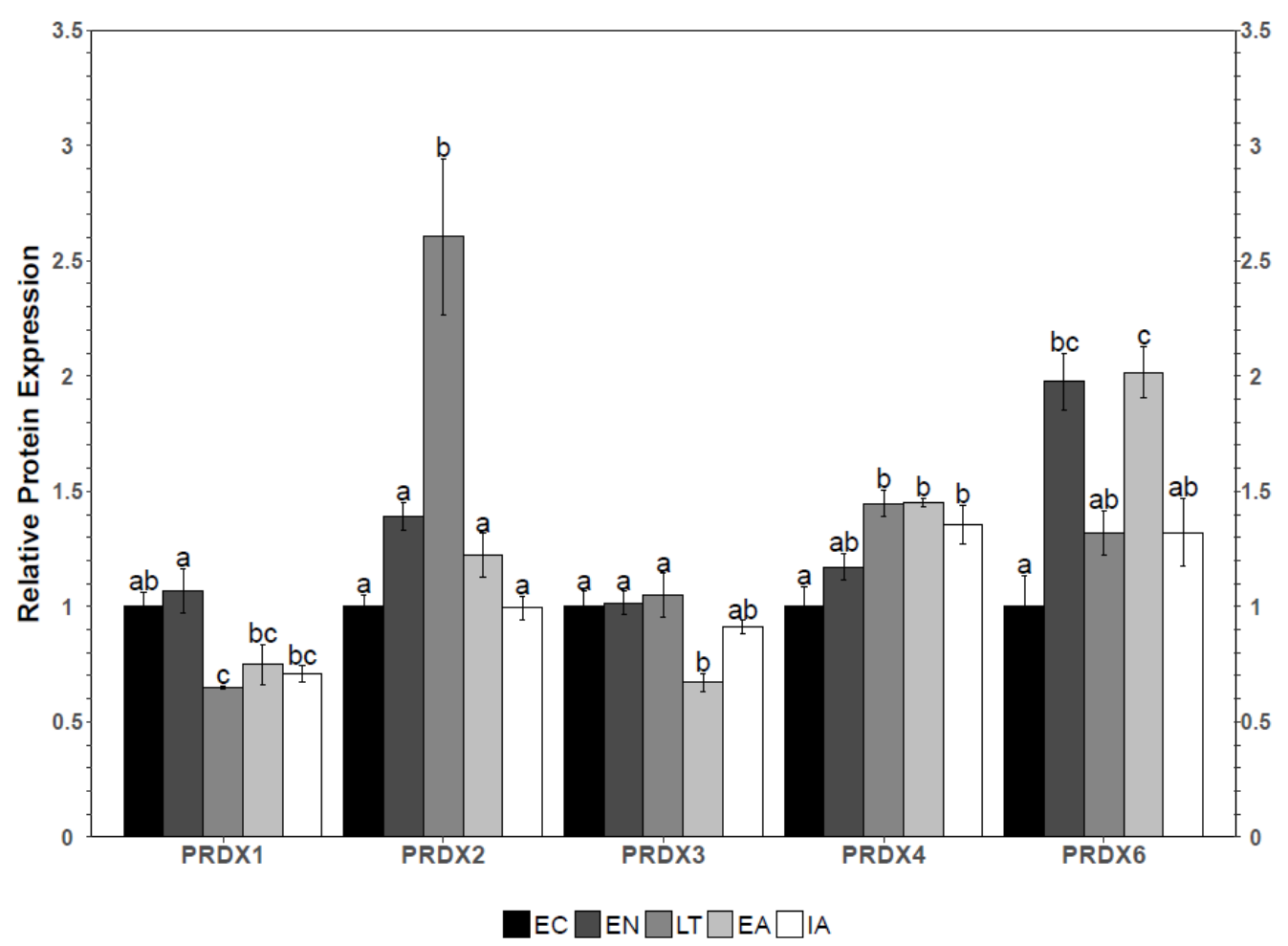

(B)

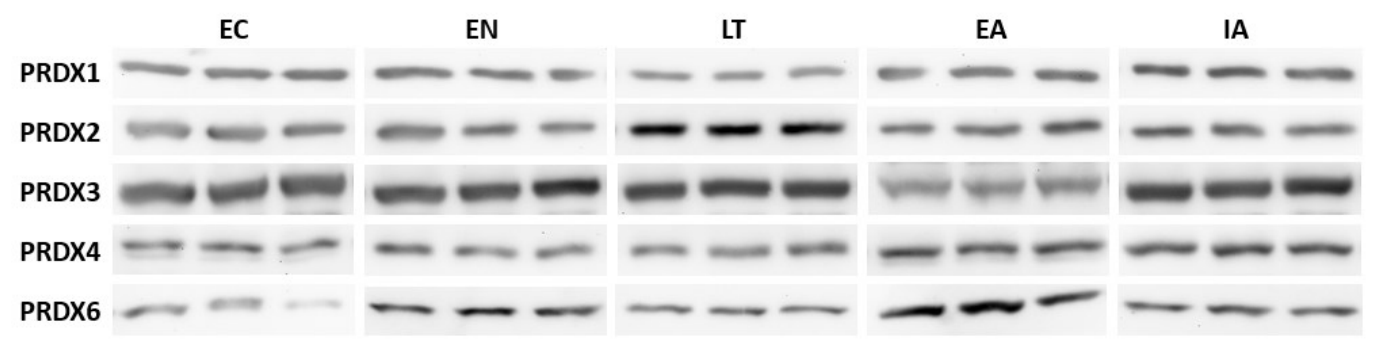

Figure 2.3: Relative protein expression of peroxiredoxin isoforms 1-4 and 6 in the liver of I. tridecemlineatus throughout the torpor-arousal cycle. (A) Histogram representing the relative protein levels using mean immunoblotting band densities. (B) Representative western immunoblot bands illustrating the expression levels of all targets. Data are mean \pm SEM ( $n=4$ independent protein isolations from different animals). Data were analyzed using an analysis of variance followed by a Tukey post-hoc test $(p<0.05)$; values sharing the same letter notation are not statistically significant from one another. 
(A)

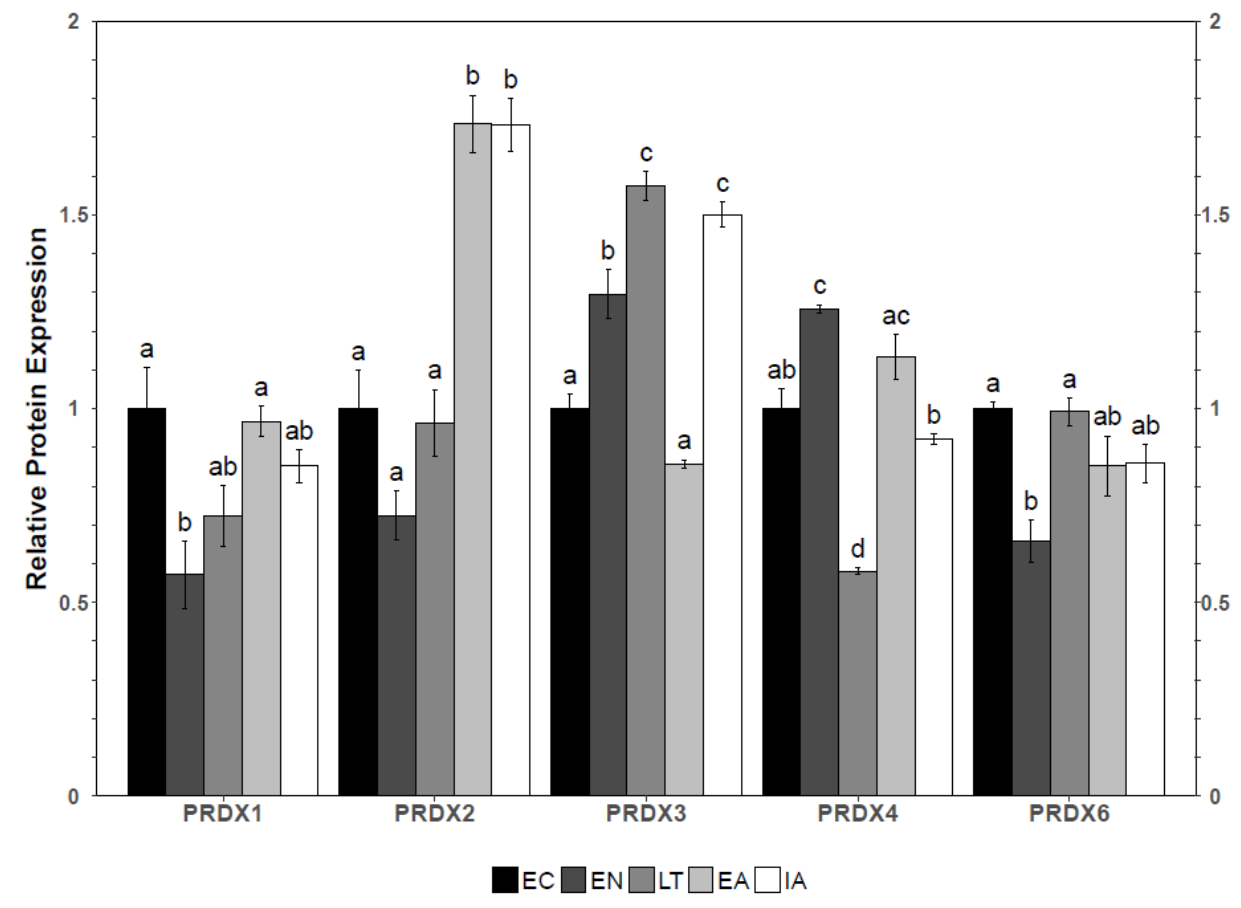

(B)

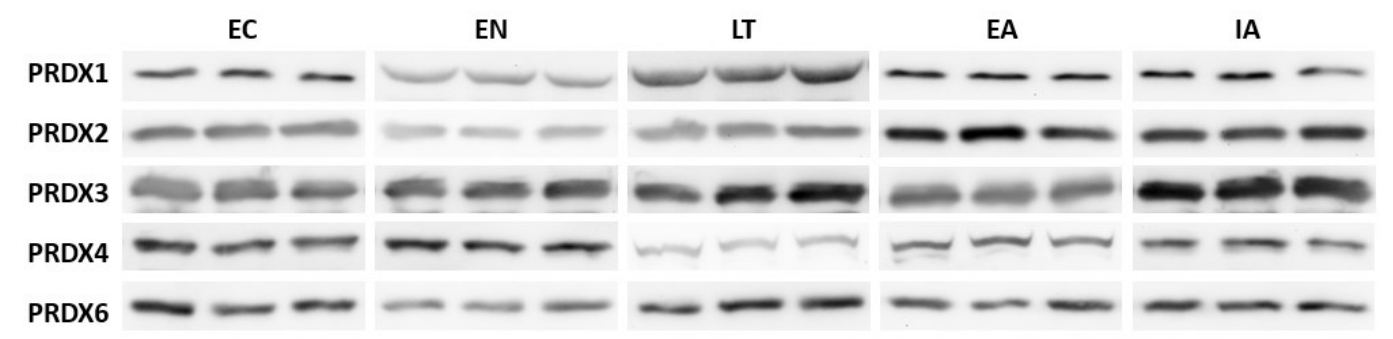

Figure 2.4: Relative protein expression of peroxiredoxin isoforms 1-4, 6 in the brown adipose tissue of I. tridecemlineatus throughout the torpor-arousal cycle. (A) Histogram representing the relative protein levels using mean immunoblotting band densities. (B) Representative western immunoblot bands illustrating the expression levels of all targets. Data are mean $\pm \operatorname{SEM}(n=4$ independent protein isolations from different animals). Data were analyzed using an analysis of variance followed by a Tukey post-hoc test $(p<0.05)$; values sharing the same letter notation are not statistically significant from one another. 
(A)

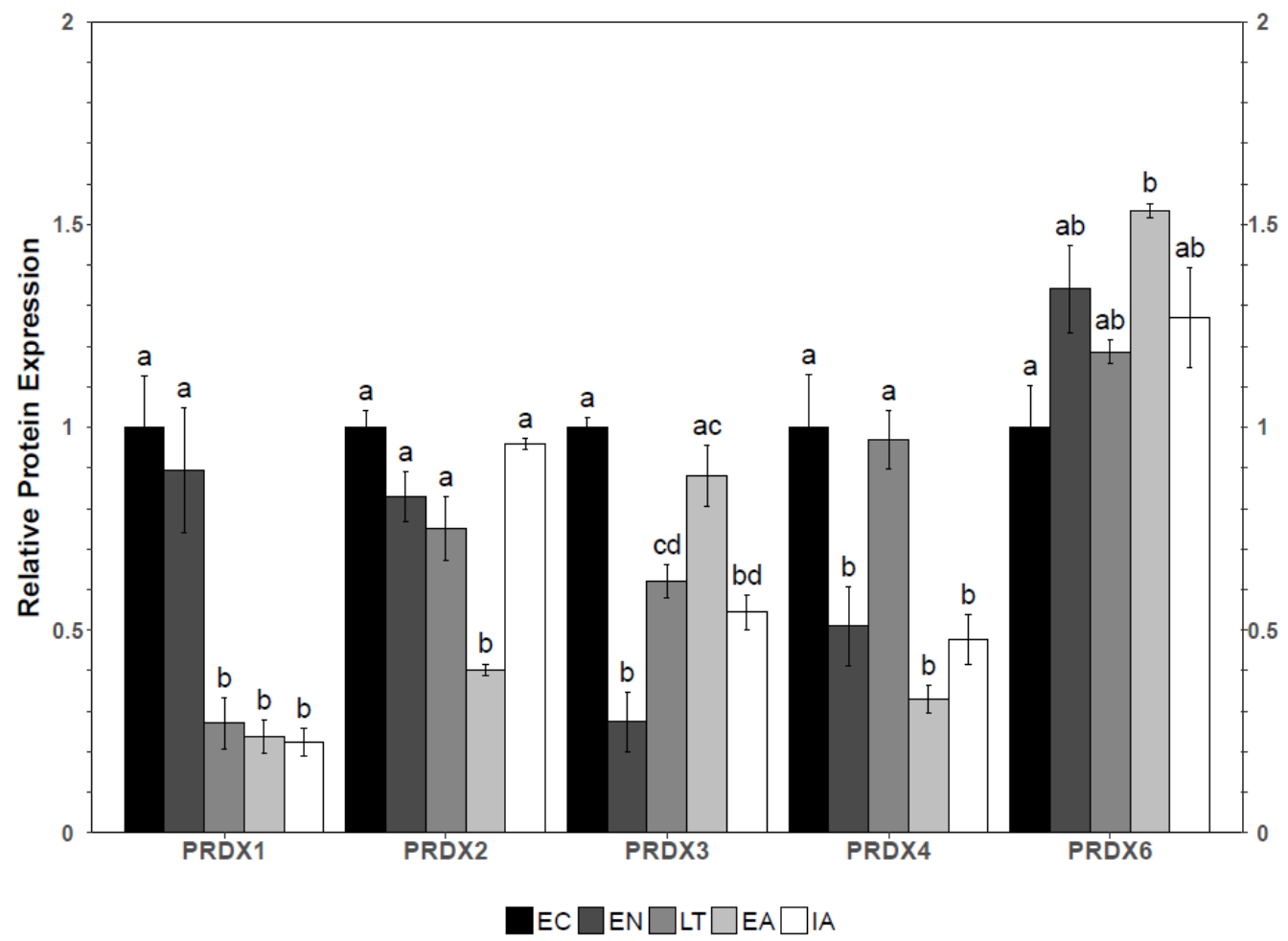

(B)

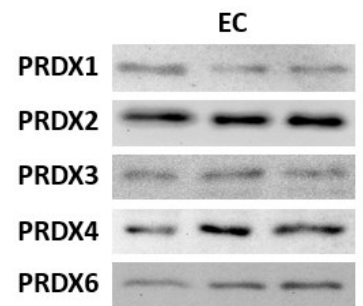

EN

LT

EA

IA
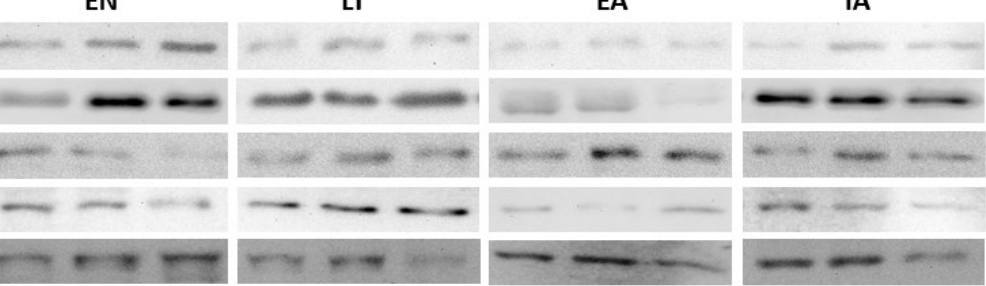

Figure 2.5: Relative protein expression of peroxiredoxin isoforms 1-4 and 6 in white adipose tissue of $I$. tridecemlineatus throughout the torpor-arousal cycle. (A) Histogram shows relative protein levels from mean immunoblot band densities. (B) Representative western immunoblot bands illustrating the expression levels of all targets. Data are mean \pm SEM ( $n=4$ independent protein isolations from different animals). Data were analyzed using an analysis of variance followed by a Tukey post-hoc test $(p<0.05)$; values sharing the same letter notation are not statistically significant from one another. 


\section{Appendix 2.1 Luminex multiplex assay}

Luminex is a multiplex magnetic bead-based multi-analyte profiling analysis method which enables the simultaneous detection and quantitation of multiple proteins at once in a 96-well plate format. This high-throughput technology produces results comparable to an ELISA, but with much greater efficiency, speed and dynamic range. (A) Illustrates the binding the binding of each bead to a capture antibody which then binds the target molecule in the sample. Subsequently, a detection antibody and streptavidin are added to quantify the amount of each specific bound bead in each well. (B) Illustrates the same binding as well as the flow cytometry technology which uses two different wavelengths of light; a red 620nm laser, excites dyes within the beads to identify the bead region, and the green $535 \mathrm{~nm}$ laser identifies the fluorescence of the reporter molecule.
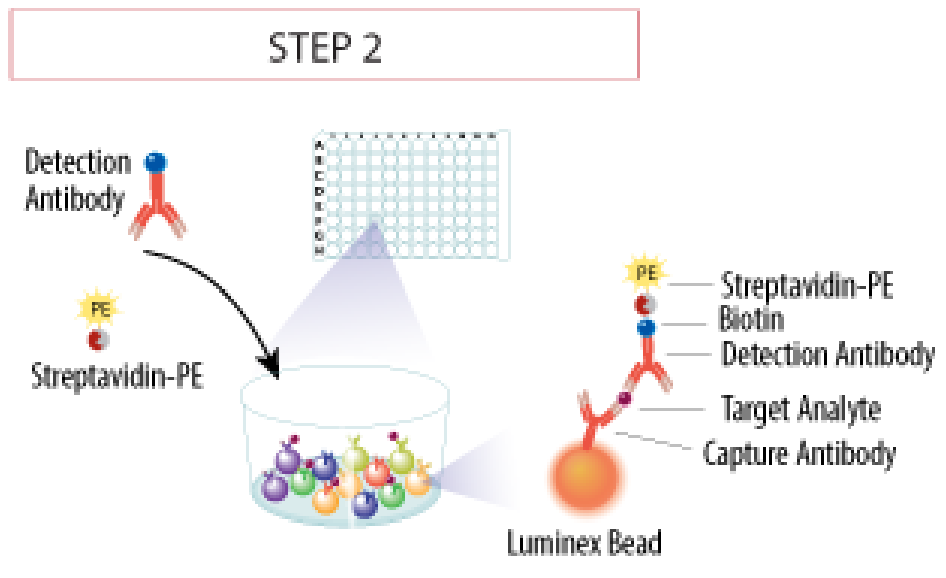

(A)
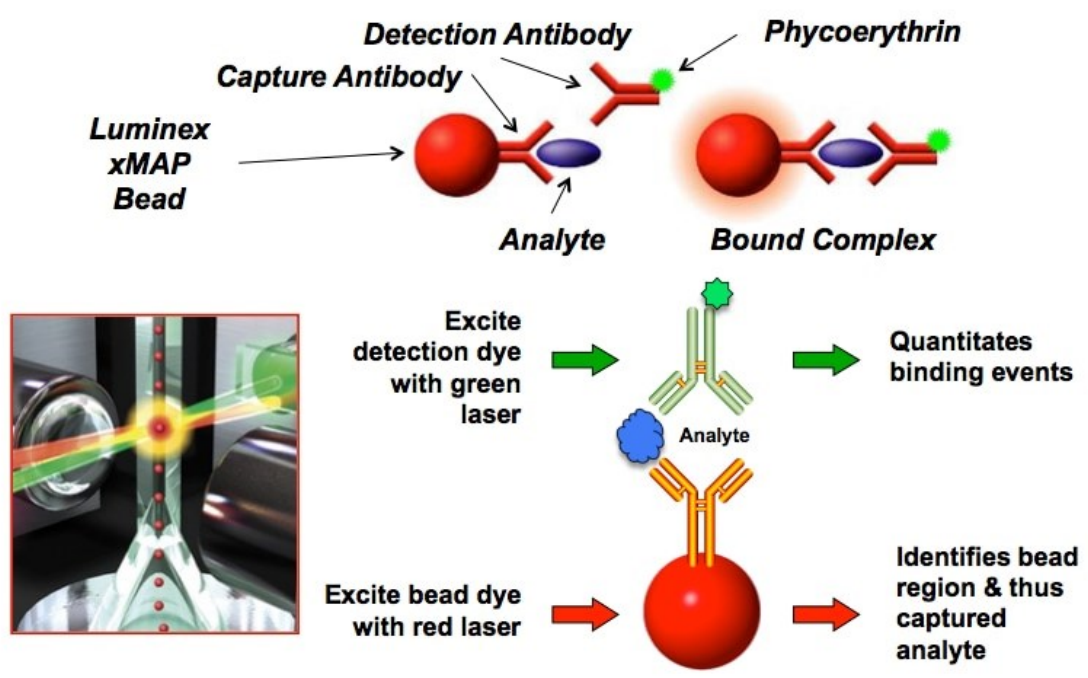

(B) 


\title{
CHAPTER 3
}

\author{
Don't go breaking my PARP - \\ How hibernating squirrels protect their DNA
}




\begin{abstract}
Some causal factors of DNA damage include the action of both exogenous agents (ionizing radiation and genotoxic chemicals) and endogenous agents (reactive oxygen species and free radicals). Through differential gene regulation, many organisms have developed specialized adaptations that enable them to survive prolonged exposures to these oxidants. One such animal is the thirteen-lined ground squirrel, Ictidomys tridecemlineatus that has developed protective mechanisms to manage ischemic stress and reperfusion injury. Research has shown that these squirrels do not accrue significant DNA damage during hibernation and thus, it is hypothesized that damage-repair pathways are being induced to mitigate this potential injury. Multiple repair pathways function in concert to modulate DNA repair mechanisms depending on the type of lesion, including those mediated by poly-(ADP)-ribosyl polymerase (PARP). In the hibernating ground squirrel, PARylation levels rose significantly in the liver to 3.90-fold over euthermic controls but showed no change in the skeletal muscle. These results were mirrored in the cyto-nuclear distribution since nuclear PARP-1 in liver increased during torpor by 1.62 -fold, indicating translocation into the nucleus, but no change was seen in the PARP cyto-nuclear levels in muscle. An important marker of DNA damage, 8-OH-dG, was elevated in the liver but suppressed in the muscle during early arousal from torpor. Downstream DNA repair proteins revealed a similar trend where a 6.68 -fold increase in $\mathrm{Ku} 80$ was noted in the liver, whereas muscle revealed an increase in Rad50 of 1.46-fold but a decrease in XRCC4-like factor (XLF) to 63\% during torpor. Upstream kinases, ATM and ATR revealed an increase in both targets to 1.40- and 5.90-fold, respectively, in liver whereas muscle tissue had a 2.43-fold increase in ATR during torpor with no significant change in ATM protein levels. Taken together, this work expands our understanding of the molecular mechanisms that control the DNA damage repair response during mammalian hibernation.
\end{abstract}




\subsection{Introduction}

\subsubsection{The thirteen-lined ground squirrel}

Hibernation is a state of heterothermy employed by various mammals to escape low winter temperatures and the food scarcity that accompanies winter. Animals such as the thirteen-lined ground squirrel enter a state of hypometabolism during which significant metabolic reorganization takes place that allows the animals to abandon the high energy costs of euthermia. During this time, squirrels cycle through prolonged periods of deep torpor where metabolic rate is suppressed to as low as $2-4 \%$ of normal euthermic values $[18,69,126]$, that are interspersed by brief periods of arousal where metabolic rate returns to euthermic values [69]. Many physiological and biochemical changes occur to facilitate metabolic rate suppression. These include a decrease in breathing rate (100-200 to 4-6 bpm), a decrease in heart rate (200-300 to 3-5 bpm), a switch from a primary reliance on carbohydrates to lipids as a primary fuel source, and the use of brown adipose tissue (BAT) and skeletal muscle to rewarm the body from near ambient $\left(0-5^{\circ} \mathrm{C}\right)$ back to euthermia $[18$, $44,69,126]$. During this rewarming period, tissues become quickly reperfused with oxygen as the squirrel elevates breathing but this also increases the production of reactive oxygen species (ROS) that can damage cells.

While ground squirrel tissues cycle through periods of ischemia, these mammals have developed protective mechanisms to avoid reperfusion injury over cycles of restricted and then restored blood flow. The fluctuations between torpor and arousal cause variances in oxygen consumption and perfusion rates of tissues resulting in the accumulation of endogenous oxidants, especially during the arousal from torpor. The ground squirrel avoids oxidative damage during the early arousal stages by upregulating pro-survival pathways (e.g. antioxidant enzymes, heat shock proteins, and anti-apoptotic agents) [7, 42, 45, 69, $119,127]$. Increased production of oxidants has long been known to be one of the causal factors of DNA damage and has been shown to be mutagenic and result in various pathologies [128-131]. Important mechanisms exist for the detection of different types of DNA damage including double and single stranded breaks. A key sensor is poly-(ADP)ribosyl (PAR) polymerase (PARP), which catalyzes the addition of PAR groups, known as 
PARylation, on histones and nuclear proteins at sites of DNA damage [132]. DNA damage leads to two kinds of PARP activation depending on the severity of the damage. Limited damage induces PARP activity which then stimulates the activation and recruitment of repair proteins. However, excessive damage or DNA damage that is left unrepaired leads to the caspase-induced cleavage of PARP. This inactivates the repair process and causes cell growth arrest or apoptosis to prevent gross chromosomal aberrations [132, 133]. PARPs constitute a large family of enzymes with $>16$ isoforms, but only PARP-1 and PARP-2 have been shown to play significant roles in damage sensing and signaling [132]. PARylation at sites of DNA damage results in the concerted actions of various effector molecules including the ATM and ATR kinases, X-ray repair cross-complementing protein (XRCC) ligases, DNA binding proteins and other repair proteins.

\subsubsection{Non-homologous end joining and homologous recombination}

The most common type of DNA lesions are single stranded breaks (SSB), where only one strand is interrupted [134]. If SSB are left unrepaired they have the potential to cause a double stranded break (DSB) making them an important checkpoint for DNA repair. Eukaryotic cells employ several processes to repair such lesions. A subset of these is non-homologous end joining (NHEJ) and homologous recombination (HR). These mechanisms repair breaks with little or no sequence homology required for the recruitment of effector molecules to the site of the break to initiate repair. During NHEJ, DNA ends are bound by the Ku70/80 heterodimer, which forms an encircling duplex around exposed DNA ends to protect them from degradation and to then recruit additional NHEJ components [135]. The Ku dimer ends are tethered by a DNA-dependent protein kinase catalytic subunit (DNA-PKcs), followed by ligation of the broken ends by the XRCC4XLF complex and DNA ligase IV. By contrast, during HR the first step of DNA repair is the binding of the DNA ends by the MRN complex (Mre11, Rad50 and Nibrin/Nbs1/P95) and CtIP in humans [136]. Once these components are bound, DNA resection can occur to generate single stranded DNA for repair. Resection involves the Mre11 nuclease and either EXO1 or DNA2 nuclease for short-range or long-range resection, respectively [134]. Importantly, to allow for sufficient time for repair, cell cycle machinery is halted. To initiate 
cell cycle arrest, the presence of broken ends will trigger the recruitment of repair enzymes, but also checkpoint machinery. DSBs activate the ATM kinase, which is also activated by the MRN complex. The ATR pathway is also activated during this time, and both sensors work to activate DNA repair machinery as well as achieve cell cycle arrest through the activation by phosphorylation of cyclin-dependent kinases (Cdks) that control cell cycle progression $[133,137]$.

\subsubsection{Markers of DNA damage - H2A.X and 8-OH-dG}

Some modifications of DNA occur so frequently that they have been termed "markers" of damage. Consistently, histone H2A.X is rapidly phosphorylated by ATM or DNA-PKcs in response to damage from chemotherapeutics, ionizing radiation, as well as oxidative stress $[138,139]$. Similarly, hydroxylation of guanosine to form 8-hydroxy-2'deoxyguanosine (8-OH-dG) or 8-oxo-7,8-dihydro-2'-deoxyguanosine (8-oxodG) often occurs as a result of hydroxylation of the DNA adduct by similar agents including free radicals and other ROS. The latter mutation has been found to be one of the most predominantly occurring lesions in response to excessive oxidative stress and is widely used as a marker of oxidative damage in various pathologies including carcinogenesis, Alzheimer's disease, stroke and cardiac arrest, among others [37, 140].

Historically, the prolonged period of mammalian hibernation showed no evidence of any significant cellular or DNA damage. The present study attempts to elucidate whether genetic damage simply does not occur, or whether cellular repair machinery is quickly turned on to mitigate any accumulating damage during the period of torpor, a time when many critical yet energy expensive mechanisms are suppressed. The status of DNA-damage repair markers was assessed, as well as the relative protein expression of various effectors responsible for relaying and activating the stress-response to DNA damage that ultimately leads to initiating repair. These were monitored in liver and skeletal muscle of $I$. tridecemlineatus over the torpor-arousal cycle. The targets examined included the relative expression levels of the multifactorial sensor of damage (PARP-1) along with its subcellular distribution, as well as effector molecules responsible for initiating the damage repair response, and a key marker of DNA damage, $8-\mathrm{OH}-\mathrm{dG}$. The data provide a novel 
overview of the DNA damage response and proposed tissue-specific repair patterns that occur during mammalian hibernation.

\subsection{Methods}

\subsubsection{Animal Protocols}

All animal experiments and tissue dissections were performed as previously described in Chapter 2. Animals were euthanized and tissues were dissected out and flash frozen immediately in liquid nitrogen. Tissues were stored at $-80^{\circ} \mathrm{C}$ until delivery to Carleton University on dry ice.

\subsubsection{Protein quantification}

\subsubsection{Total protein isolation}

Total protein extracts were prepared as described in Chapter 2. Briefly, $100 \mathrm{mg}$ of samples were homogenized 1:4 w:v using a Polytron PT10 and homogenizing buffer (20 mM Tris base, $150 \mathrm{mM} \mathrm{NaCl}, 1 \mathrm{mM}$ EDTA, $1 \mathrm{mM}$ EGTA, $1 \mathrm{mM} \mathrm{NaF}, 10 \mathrm{mM} \beta-$ glycerophosphate, $1 \% \mathrm{v} / \mathrm{v}$ Triton $\mathrm{X}-100$, and $1 \mathrm{mM}$ phenylmethylsulfonyl fluoride). Samples were centrifuged at $10,000 \mathrm{x} \mathrm{g}$ for $10 \mathrm{~min}$ at $4^{\circ} \mathrm{C}$, then supernatants were removed and soluble protein concentration was determined using the Bio-Rad prepared reagent (BioRad Laboratories, Hercules, CA). Sample concentrations were adjusted to 10 $\mu \mathrm{g} / \mu \mathrm{L}$ by additions of calculated small volumes of homogenization buffer and measured aliquots were mixed 1:1 with $2 \mathrm{X}$ SDS loading buffer (100 mM Tris base $\mathrm{pH} 6.8,4 \% \mathrm{w} / \mathrm{v}$ SDS, $20 \% \mathrm{v} / \mathrm{v}$ glycerol, $0.2 \% \mathrm{w} / \mathrm{v}$ bromophenol blue, and $10 \% \mathrm{v} / \mathrm{v} 2$-mercaptoethanol), to yield a final protein concentration of $5 \mu \mathrm{g} / \mu \mathrm{L}$. Samples were boiled for 10 minutes and then stored at $-20^{\circ} \mathrm{C}$ until use.

\subsubsection{Cyto-nuclear extract preparation}

Cytoplasmic and nuclear protein extracts were prepared as per [124, 141]. Briefly, $500 \mathrm{mg}$ tissue samples from each condition were added to $1 \mathrm{~mL}$ of homogenization buffer (10 mM HEPES, pH 7.9, 10 mM KCl, 10 mM EDTA, 10 mM DTT, $1.5 \mu$ l Sigma protease inhibitor cocktail) and tissue was disrupted with approximately 20 piston strokes of a 
Dounce homogenizer. Samples were centrifuged at 10,000 RPM for $10 \mathrm{~min}$ at $4^{\circ} \mathrm{C}$ and then the supernatants were removed as the cytoplasmic fractions. Next, pellets were resuspended in extraction buffer (20 mM HEPES, pH 7.9, $400 \mathrm{mM} \mathrm{NaCl}, 1 \mathrm{mM}$ EDTA, $10 \% \mathrm{v} / \mathrm{v}$ glycerol, $10 \mathrm{mM}$ DTT, $1.5 \mu \mathrm{l}$ protease inhibitor cocktail) and $150 \mu \mathrm{L}$ of extraction buffer was added per gram of starting material. Samples were placed on ice and rocked for $1 \mathrm{~h}$ on a rocking platform with mixing every $15 \mathrm{~min}$. Samples were then centrifuged at 10,000 RPM for $10 \mathrm{~min}$ at $4^{\circ} \mathrm{C}$ and the supernatant was removed and placed into new sterile tubes. This supernatant contained the nuclear fraction. Soluble protein concentrations of both cytoplasmic and nuclear fractions were quantified using the BioRad assay. Protein concentrations were standardized to $4 \mu \mathrm{g} / \mu \mathrm{L}$ and then aliquots were mixed 1:1 with $2 \mathrm{X}$ SDS loading buffer to yield final protein concentrations of 2 $\mu \mathrm{g} / \mu \mathrm{L}$. Cyto-nuclear extractions were validated by running samples on a SDS-PAGE and probing for a protein located only in the nucleus (Histone H2) (Cell Signaling Cat\#4499).

\subsubsection{Total Protein Immunoblotting}

Total protein lysates were prepared and immunoblotted as previously described in Chapter 2 and [45]. Briefly, 20-30 $\mu$ g of protein was loaded into each well of 8-12\% SDSPAGE gels and run for $45-60 \mathrm{~min}$ at $180 \mathrm{~V}$. After transfer to PVDF for $90 \mathrm{~min}$ at $160 \mathrm{~mA}$, membranes were blocked using 1-2\% skim milk in TBST for $30 \mathrm{~min}$ and were then probed with respective primary antibodies overnight at $4^{\circ} \mathrm{C}$. All antibodies were from Cell Signaling: PARP-1 (Cat \#9542), XLF (Cat \#2854), Ku80 (Cat \#2180), Mre11 (Cat \#4847), p-Mre11Ser676 (Cat \#4859), Rad50 (Cat \#3427), p-p95/Nibrin (Cat \#3001) ATM (Cat\#2873), and ATR (Cat\#13934). Next, membranes were probed with secondary antirabbit IgG HRP-linked antibody (diluted 1:6000 in TBST) for $30 \mathrm{~min}$ at room temperature on a plate rocker.

\subsubsection{Quantification and Data Analysis}

Bands densities were visualized with enhanced chemiluminescence using a ChemiGenius Bio-Imager (Syngene, Frederick, MD) and quantified by densitometric analysis using GeneTools Software. Band intensity of each lane was standardized against 
the summed intensity of a group of Coomassie-stained bands in the same lane to correct for variation in sample loading. Mean normalized band densities \pm SEM were calculated for control and torpor samples with $n=4$ independent tissue extracts from different animals. Data were analyzed using a Student's t-test to identify significant differences between the groups (control vs. torpor) with significance accepted if $p<0.05$. For each figure, values that are statistically significant from control have an asterisk (*) or have different letter notation. All statistical analysis and figure generation was performed using RBioplot [104].

\subsubsection{Oxidative Damage ELISA Assay}

DNA was prepared according to instructions in the ZYMO Genomic DNA tissue MiniPrep kit (Zymo Cat\# D4068). Briefly, $25 \mathrm{mg}$ tissue samples were each combined with a solution of Solid tissue buffer and Proteinase K and left to incubate overnight at $55^{\circ} \mathrm{C}$. Next, two volumes of Genomic Binding Buffer were added to samples and vortexed thoroughly. The mixture was transferred to a Zymo-Spin ${ }^{\mathrm{TM}}$ IIC-XL Column in a collection tube and then samples were centrifuged at $12,000 \mathrm{x} g$ for $1 \mathrm{~min}$. The collection tube with the flow through was discarded. A $400 \mu \mathrm{L}$ aliquot of DNA Pre-Wash Buffer was then added to each tube and was spun again as above. Next, two washes with g-DNA Wash buffer were done with 700 and $200 \mu \mathrm{L}$ of solution. Finally, the spin column was transferred to a clean microcentrifuge tube and $90 \mu \mathrm{L}$ of DNA Elution Buffer was added to the matrix. Samples were left to incubate for $5 \mathrm{~min}$ at room temperature and were centrifuged at 12,000 x $g$ for 1 min to elute the DNA. DNA was stored at $-20^{\circ} \mathrm{C}$ until use. DNA concentrations were determined using a Take3 micro-volume quantification plate (BioTek) and PowerWave HT Spectrophotometer (BioTek). Purity of samples was assessed using the 260/280 nm ratio as well as assessing the presence of sharp bands for $28 \mathrm{~S}$ and $18 \mathrm{~S}$ ribosomal RNA using 1\% agarose gel electrophoresis. DNA samples were standardized to $30 \mathrm{ng} / \mu \mathrm{L}$ using autoclaved water and kept on ice. DNA was digested using 5 Units of nuclease P1 (Sigma Cat\# N8630$1 \mathrm{VL}$ ) by incubating samples at $55^{\circ} \mathrm{C}$ for $1 \mathrm{~h}$. Next, 1 unit of Alkaline Phosphatase (NEB, Cat\# M0290S) was added and samples were incubated at $37^{\circ} \mathrm{C}$ for $30 \mathrm{~min}$, then boiled for $10 \mathrm{~min}$ and placed on ice until use. 
All reagents and standards were prepared according to manufacturer's instructions for the DNA/RNA Oxidative Damage ELISA kit (Cayman Cat \# 589320). Briefly, standards were prepared from stock reagents supplied in the kit for the preparation of 7 serial dilutions from reconstituted standard $(10.3-3000 \mathrm{pg} / \mathrm{mL})$. Blanks included a nonspecific binding (NSB) blank (100 $\mu$ L ELISA buffer, $50 \mu \mathrm{L}$ of tracer dye), a maximum binding $\left(\mathrm{B}_{\mathrm{o}}\right)$ blank $(50 \mu \mathrm{L}$ of ELISA buffer, $50 \mu \mathrm{L}$ of tracer, and $50 \mu \mathrm{L}$ of monoclonal antibody) and a blank (Blk) for background absorbance caused by the Ellman's reagent. For the assay, $150 \mu \mathrm{L}$ of DNA sample (50 $\mu \mathrm{L}$ sample, $50 \mu \mathrm{L}$ tracer, and $50 \mu \mathrm{L}$ antibody) from EC, LT and EA conditions, as well as standards and blanks, were loaded into wells of the supplied microplate, covered with an adhesive strip and incubated overnight in the dark on a plate shaker. The next morning, each well was washed 5 times with wash buffer, incubated with $200 \mu \mathrm{L}$ of Ellman's reagent, and allowed to develop in the dark for $120 \mathrm{~min}$ on a plate shaker at room temperature. Optical densities were measured spectrophotometrically at $420 \mathrm{~nm}$ with blank subtraction. Mean standardized values for 8$\mathrm{OH}-\mathrm{dG}$ abundance \pm SEM ( $\mathrm{n}=4$ independent replicates from different animals) were calculated. Data were analyzed using a one-way ANOVA followed by a Tukey post-hoc test to identify significant differences between conditions $(p<0.05)$. All statistical analysis and figure generation was performed using RBioplot [104]. 


\subsection{Results}

\subsubsection{8-hydroxy-deoxyguanosine - A Marker of Damage}

The abundance of the DNA damage marker 8-OH-dG was examined in both tissues during late torpor as well as early arousal and compared to control values (Fig 3.1). Results revealed differential tissue-specific expression where the liver showed no significant change between euthermic control (EC) and late torpor (LT) but revealed a slight increase during early arousal (EA) to $1.10 \pm 0.01$-fold which was significantly elevated from LT, but not from EC. In contrast, skeletal muscle showed a downward trend in LT to $68 \% \pm 4 \%$ of the EC value that was not significantly different from controls, but was followed by a further significant decrease to $0.39 \pm 0.01$ of the EC value during EA.

\subsubsection{ATM/ATR kinases}

ATR and ATM protein levels showed differential expression between euthermic control and late torpor conditions (Fig 3.2). In the liver, a $1.40 \pm 0.04$-fold significant increase in ATM content occurred during torpor whereas skeletal muscle showed no change. However, protein levels of the ATR kinase showed strong significant increases in both liver and muscle relative protein expression during torpor to $5.90 \pm 0.27$-fold and $2.43 \pm 0.31$-fold higher, respectively, than euthermic control values.

\subsubsection{PARP-1}

Upon sensing damage, PARP-1 shuttles into the nucleus from the cytoplasm and binds to sites of DNA damage, followed by recruiting other repair enzymes. To elucidate its subcellular localization during hibernation, nuclear and cytoplasmic fractions were isolated from liver and skeletal muscle and PARP-1 was measured in both fractions. Total PARP-1 protein levels increased strongly in liver by $3.92 \pm 0.30$-fold during torpor as compared to control values (Fig 3.3A). PARP-1 in the nucleus also increased during torpor by $1.62 \pm 0.07$-fold compared to euthermic controls. Correspondingly, cytoplasmic PARP1 expression decreased during torpor to $43 \pm 3 \%$ of the control value. In skeletal muscle,

the response was different. Both total and cytoplasmic PARP-1 protein expression was unchanged between control and torpor conditions, but a decrease in PARP-1 protein content in the nuclear fraction was noted, levels falling to $53 \pm 8 \%$ of control values (Fig 3.3B). 


\subsubsection{DNA damage repair targets}

Finally, HR and non-homologous end joining targets were examined in the liver (Fig 3.4A) and the skeletal muscle (Fig 3.4B) from euthermic control and late torpor ground squirrels. Of these, several targets revealed differential tissue-specific regulation during torpor compared to their respective euthermic values. Ku80 was strongly elevated in liver during torpor by $6.68 \pm 1.03$-fold as compared to euthermic values, whereas the skeletal muscle showed no significant change in Ku80 protein. In the liver, XLF was suppressed to $33 \pm 6 \%$, and in the muscle was suppressed to $63 \% \pm 4 \%$ compared to euthermic values. Rad50 did not change in the liver during torpor, but a significant increase was noted in the skeletal muscle to $1.46 \pm 0.10$-fold above euthermic control values. Levels of phosphorylated P95 (Nibrin) (Ser343) did not change significantly in liver or skeletal muscle in response to torpor, although a downward trend was noted in skeletal muscle. Total Mre11 and phosphorylated Mre11 (Ser676) revealed differential expression. Neither tissue showed a significant change in total Mre11 levels, but a significant decrease in phospho-Mre11 (Ser676) occurred in liver during torpor, levels falling to $0.54 \pm 0.06$ as compared with controls. In the skeletal muscle, the opposite occurred with a strong $2.64 \pm$ 0.29 -fold increase in the phosphorylation status of the protein during torpor.

\subsection{Discussion}

The integrity of a cell's genome is challenged every day by intrinsic and extrinsic stressors. In order to maintain genetic integrity, cells must possess a highly organized mechanism for repairing damage to DNA. This response includes the halting of cell-cycle progression in order to allow for the repair of DNA breaks or invoking apoptosis if damage is excessive. The importance of this mechanism is illustrated by the presence and variety of diseases linked to the damage response. Repair is controlled like a signaling pathway; activated by aberrant DNA structures, and recognized by sensor molecules that relay the information to effectors, that activate the aforementioned pathways. Interestingly, a tissuespecific, as well as a possible damage-specific, response was observed in the ground squirrel with the more metabolically active liver demonstrating a stronger regulation of 
DNA repair compared to the more quiescent muscle. We describe the detailed regulation of the damage response in the context of hibernation, a time during which hypometabolism prioritizes pathways and tissues critical for survival.

Many mammalian hibernators endure severe hypothermia during the winter followed by periodic rewarming back to euthermia. The decrease in body temperature to near ambient levels is one of the main contributors of the maintenance of cellular integrity. Large hibernators such as the black bear can suppress their metabolism to as low as $25 \%$ of basal rates, as well as decrease their body temperature to $30-33^{\circ} \mathrm{C}$ [142] whereas in humans, a decrease in body temperature by a couple of degrees (to $33-35^{\circ} \mathrm{C}$ ) is enough to induce potentially lethal consequences of hypothermia. The ability of the 13-lined ground squirrel to suppress its metabolism by over $95 \%$ of euthermic values is strongly dependent on its concomitant ability to reduce its body temperature to staggeringly low levels (often just $3-5^{\circ} \mathrm{C}$ ). Interestingly, this natural model of seasonal hypothermia has been modelled for the treatment of various medical conditions including traumatic brain injuries, asphyxia, cardiac arrest and several forms of ischemic injuries [143, 144] and has resulted in remarkable recoveries with markedly reduced cellular injury.

\section{8-hydroxy-2'-deoxyguanosine}

During the early stages of arousal, when high levels of reactive oxygen species begin to plague the cell, DNA modifications, such as hydroxylations, are more likely to occur $[1,145]$. Interestingly, $8-\mathrm{OH}-\mathrm{dG}$ levels in liver showed no significant changes between euthermia and prolonged torpor, but increased by approximately $10 \%$ during early arousal, a time during which oxidants are known to peak [146]. Conversely, the levels of 8-OH-dG in skeletal muscle revealed a downward trend during late torpor and were decreased significantly by over 50\% compared to controls during early arousal (Fig 3.1). This could suggest that the liver may be experiencing more DNA damage that is constantly being fixed whereas the muscle may not be incurring the same levels of damage or is removing it fast. This could be due to the fact that the liver is a much more metabolically active organ responsible for activating various pro-survival pathways such as apoptosis, the 
heat-shock protein response, the antioxidant response, and protein folding among others [7, $147,148]$ whereas the muscle is fairly quiescent during hibernation.

Both human and rodent studies have shown elevated levels of $8-\mathrm{OH}-\mathrm{dG}$ during periods of ischemic stress, such as stroke, but also in cases of increased risk of cancer, coronary heart disease, diabetes and atherosclerosis as well as in cases of hypertension and in atherosclerotic plaques [149-151]. These are tissue-specific diseases which suggest that more metabolically or physiologically active tissues are more likely to succumb to damage than more quiescent tissues such as the skeletal muscle that are recruited upon need/demand. This idea fits with the data from the present research in that putting organs under specific conditions that cause undue stress for extended periods of time, may be putting excessive strain on those tissues and thereby result in accumulation of damage markers or a reduction in the speed of repair. Therefore, in response to these stresses, detectable levels of 8-OH-dG can be measured in these tissues, blood, or in urine into which $8-\mathrm{OH}-\mathrm{dG}$ is excreted.

Interestingly, the application of mild hypothermia to a rat model of cerebral ischemia using middle cerebral artery occlusion was seen to confer robust neuroprotection. This was characterized by decreased infarct volume, reduced levels of DNA lesions (including 8-OH-dG), apurinic/apyrimidinic (AP) sites, and single stranded breaks as well as the suppression of pro-apoptotic targets [152] suggesting that a decreased body temperature may reduce or slow damaging effects. Therefore, the reduced body temperature of the hibernating squirrel appears to be cytoprotective against increasing levels of 8-OH-dG during arousal in the liver and is perhaps accompanied by an increase in antioxidant enzymes and repair proteins. This is further supported by several studies, which show an elevation of antioxidant enzymes during torpor and/or the recovery periods of hypometabolism, including hibernation [7, 153-155]. Skeletal muscle, on the other hand, shows a decrease in this modification. It is possible that because the skeletal muscle is father away from the body's core than the liver, it gets even colder during hibernation than the liver, and therefore evades higher levels of damaging agents. Unfortunately, this hypothesis has yet to be tested and more studies in this area are needed. 
Upstream ATM and ATR kinases

The sensors of the damage-response signaling cascade, such as PARP and the MRN complex, detect DNA strand breaks or replication stress. However, transducers, such as ATM and ATR kinases activate effectors to carry out the repair response as well as initiate other survival pathways, and the effectors carry out a broad range of processes important for maintenance of cellular integrity [156]. The two transducers, ATM and ATR, are responsible for phosphorylating downstream damage targets as well as activating cell-cycle checkpoints $[156,157]$. It has been noted that both ATM and ATR are activated in response to DNA damage and replication stress and although they often work together to regulate similar processes, their specificities are distinct. Hundreds of proteins are phosphorylated by these two transducers at Ser-Thr/Glu motifs whereas DNA-PKcs regulate a smaller number of targets $[36,139]$. Activation of ATM by DSBs leads to the phosphorylation of targets such as Brca1, Chk2, and p53, among others, that mediate DNA repair, cell-cycle arrest, apoptosis and other downstream pathways [156, 158]. Incidentally, of these, Chk2 was found to be upregulated in the liver during early arousal, and p53 during late torpor, suggesting a possible mechanism of activation of these targets by ATM $[137,159]$. The MRN complex is a potent activator and recruiter of ATM to sites of DSBs and is sufficient to stimulate the kinase on its own [160]. ATR on the other hand, is activated by a broad spectrum of DNA damage in addition to DSBs. Localization of ATR to sites of damage is dependent on its interaction with ATRIP which is generally located on long stretches of ssDNA breaks, suggesting that single stranded breaks are key to activation of ATR [161]. Incidentally, previous work on a South American marsupial hibernator also found a strong upregulation of liver ATR as well as other stress-sensitive cell cycle regulators suggesting a possible hibernation specific regulation of these pathways [162]. Other studies include work on the anoxia-tolerant turtle which have shown that ATM levels increase significantly after 5 or 20 hours of anoxia exposure in liver but decrease in muscle after $5 \mathrm{~h}$ and return to control levels after $20 \mathrm{~h}$ of anoxia. The ATR kinase, on the other hand, showed no change in muscle but decreased significantly after 20 hours of anoxia in turtle liver (Biggar, 2008, unpublished results). This work suggested a stronger stress response to activate downstream cell cycle checkpoint proteins in the anoxic liver via ATM than ATR. Another 
study confirmed cell cycle arrest during torpor and re-initiation during arousal [137]. This was potentially due to the actions of one or both of these kinases. Therefore, cell cycle arrest and potentially the DNA damage response by extension, appear to be regulated in a tissue-specific manner by the action of the ATM/ATR kinases.

The current study revealed ATM and ATR kinases are activated in a tissue-specific manner with an increase seen in both the kinases in the liver and only ATR in muscle (Fig 3.2). Since liver is known to be essential for various vital metabolic functions and is a main site for pro-survival protein synthesis [163], it is possible that it is under greater metabolic demands and is subject to a higher threat of DNA damage - for both DSBs and SSBs. Therefore, an ATM-dependent cell cycle arrest is likely induced to enable repair.

\section{DNA damage repair}

In the current study, the effects of several non-homologous end-joining and homologous-recombination targets in control and late torpor conditions were also examined. A key enzyme in this pathway, PARP-1, was differentially regulated during torpor in a tissue-specific manner. Skeletal muscle showed no significant change in either in PARP-1 content in either cytosolic or nuclear fractions, but the liver revealed an increase in total and nuclear PARP levels suggesting that PARP-1 was shuttled into the nucleus during torpor (Fig 3.3A and 3.3B). Although PARP-1 can be found in both the cytoplasm and the nucleus, an increase in nuclear abundance is indicative of DNA damage sensing. PARP-1 becomes activated upon binding SSBs and PARylates a variety of targets to promote the accumulation of repair proteins and subsequently acts as a scaffold to assemble repair factors at the site of the break $[164,165]$. Also, depending on the amount of DNA damage (dictated by the amount that can be repaired efficiently), PARP-1 will act as a survival factor involved in DNA damage repair, whereas excessive damage will result in PARP-1 promoting cell death [165]. In the latter case, an $116 \mathrm{kDa}$ PARP-1 is cleaved between Asp214 and Gly215 into an amino terminal domain (24 kDa) and a carboxyterminal domain $(89 \mathrm{kDa})$ by Caspase-3. This cleavage facilitates cellular disassembly and initiates apoptosis [166]. Incidentally, the levels of cleaved PARP-1 have been measured in squirrel skeletal muscle and were seen to decrease to $60 \%$ during late torpor [127], indicating that apoptosis was not induced. Cleaved PARP-1 has a reduced affinity for DNA 
which is thought to contribute to a reduced PARylation capacity [166]. Therefore, since this cleavage does not occur in ground squirrels the nuclear localization of PARP-1 likely has other non-apoptotic functions.

The levels of individual repair enzymes were also measured. Polymorphisms in DNA repair genes are commonly found to be associated with genomic instability and various cancers. Numerous studies have shown that targets such as OGG1, BRCA2, XRCC1, XRCC3, among others, are dysregulated in adult gliomas, bladder cancers, skin cancer, breast cancer, prostate cancer, lung cancer, etc. [167]. During torpor, DNA repair targets were differentially regulated in both tissues. Ku80 increased in the liver but showed no change in the muscle, whereas Rad50 and p-Mre11 increased in muscle but not in liver (Fig 3.4A and 3.4B). Conversely, p-Mre11 decreased in the liver as well as XLF, which also decreased in muscle. Both Ku80 and Rad50 bind to the sites of DNA strand breaks in their respective DNA repair pathways (NHEJ and HR, respectively) and hold them in close proximity awaiting resection by additional repair proteins (as in the case of Ku80) or while sequence homology in DNA templates is found (as in the case of Rad50) [168]. Interestingly, the XLF ligase, was suppressed during late torpor, suggesting that binding of sensor enzymes to the sites of breaks is not suppressed, but no actual ligation/repair of broken ends occurs at this time. We postulate that perhaps ligation is a more significant energy-consuming process and possibly is induced during the arousal stages. XLF forms a dimer with XRCC4 which bridges DNA ends for ligation. Mutation of XLF inhibits this interaction and prevents NHEJ repair [169]. Interestingly, the AKT kinase is known to phosphorylate XLF at Thr181 and trigger its dissociation from the DNA ligase/XRCC4

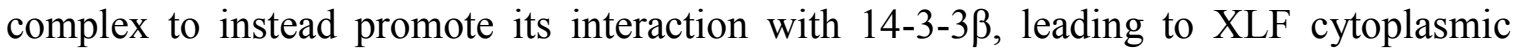
retention and subsequent degradation by SCF [170]. Incidentally, AKT has in fact been shown to be upregulated in the liver but not the skeletal muscle of hibernating ground squirrels, which offers a potential explanation for the suppression of XLF in the liver [102, $171]$.

Site-specific phosphorylation of Mrel1 
Another important target examined in this study was the phosphorylation status of Mre11 during torpor. Mre11 is a key component of the MRN complex acting as a damage sensor of double stranded breaks, but also, a multi-faceted enzyme with highly specific nuclease activity [172]. A significant reduction in phospho-Mre11 was seen in liver whereas a large induction was noted in the muscle (Fig 3.4A and 3.4B). Phosphorylation of Mre11 seems to have various effects depending on site specificity and the degree of damage. The MRN complex is responsible for the activation of ATM, which once activated, in turn phosphorylates Rad50 and Nbs1, phosphorylations that are important for cell cycle control, DNA repair and cell survival. However, a study noted that Mre11 is also phosphorylated by ATM at S676 and S678 in response to agents that induce DSBs, is dependent on the presence of Nbs1, and does not affect the association of members of the MRN complex, or ATM activation [173]. Therefore, Mre11 phosphorylation by ATM at these two sites acts as the switch that controls the extent of resection by Exonuclease 1 during $[173,174]$, suggesting that these phosphorylation sites are key to the induction of damage repair and cell survival.

Interestingly, another member of the MRN complex, Nibrin, was also found to be phosphorylated in response to irradiation, however its binding affinity to the MRN complex was unaffected [175]. It has been concluded that ATM phosphorylation of Nibrin during S-phase checkpoint activation likely occurs after the Mre11 complex has associated with damaged DNA. Later studies showed that hyperphosphorylation of Mre11 inactivated the MRN complex, and facilitated its disassociation from chromatin, resulting in the downregulation of DNA damage signaling during cell cycle checkpoint recovery [173, 174]. Therefore, the effects of phosphorylation of Mre11 appeared to be site-specific and suggested that perhaps another kinase was responsible for the site-specific phosphorylation and inactivation of Mre11. Incidentally, Polo-like kinase 1 (Plk1) (another mitotic kinase), was found to phosphorylate Mre11 at Ser649, primed subsequent phosphorylation events (at Ser688), and inhibited the loading of the MRN complex onto damaged DNA, halting repair [176]. These results support the notion that activation of DNA repair by Mre11 is in fact site specific and phosphorylation at Ser676 is a good predictor of damage repair. This corresponds to the reduced biomarkers in muscle which show a significant increase in 
muscle p-Mre11 thus suggesting initiation of repair and a reduction in the DNA damage marker 8-OH-dG. This could be supporting evidence for the induction of a repair response in the skeletal muscle.

Overall, this current study elucidates the potential regulation of DNA repair mechanisms during a hibernation bout. The findings suggest a tissue-specific regulatory pattern possibly due to the increased frequency of a specific type damage in a more metabolically active liver tissue compared to that of a less metabolically active muscle tissue. A possible increased incidence of single stranded breaks in muscle as evidenced by the increased activation of ATR kinase and p-Mre11 during torpor is also suggested. As well, a suggested increase in single stranded breaks and hydroxylation of guanine in the liver tissues is evidenced by the increase in ATR kinase and PARP-1 expression and increased 8-OH-dG during arousal. This study suggests that DNA damage repair is highly specific to the type of lesion or modification and the squirrel likely recruits various effector molecules to conserve energy in the most efficient way possible. Future work to elucidate the whole picture of damage repair during times of environmental stress, and more specifically oxidative stress, are pivotal to the treatment of various pathologies. 


\section{Figures}

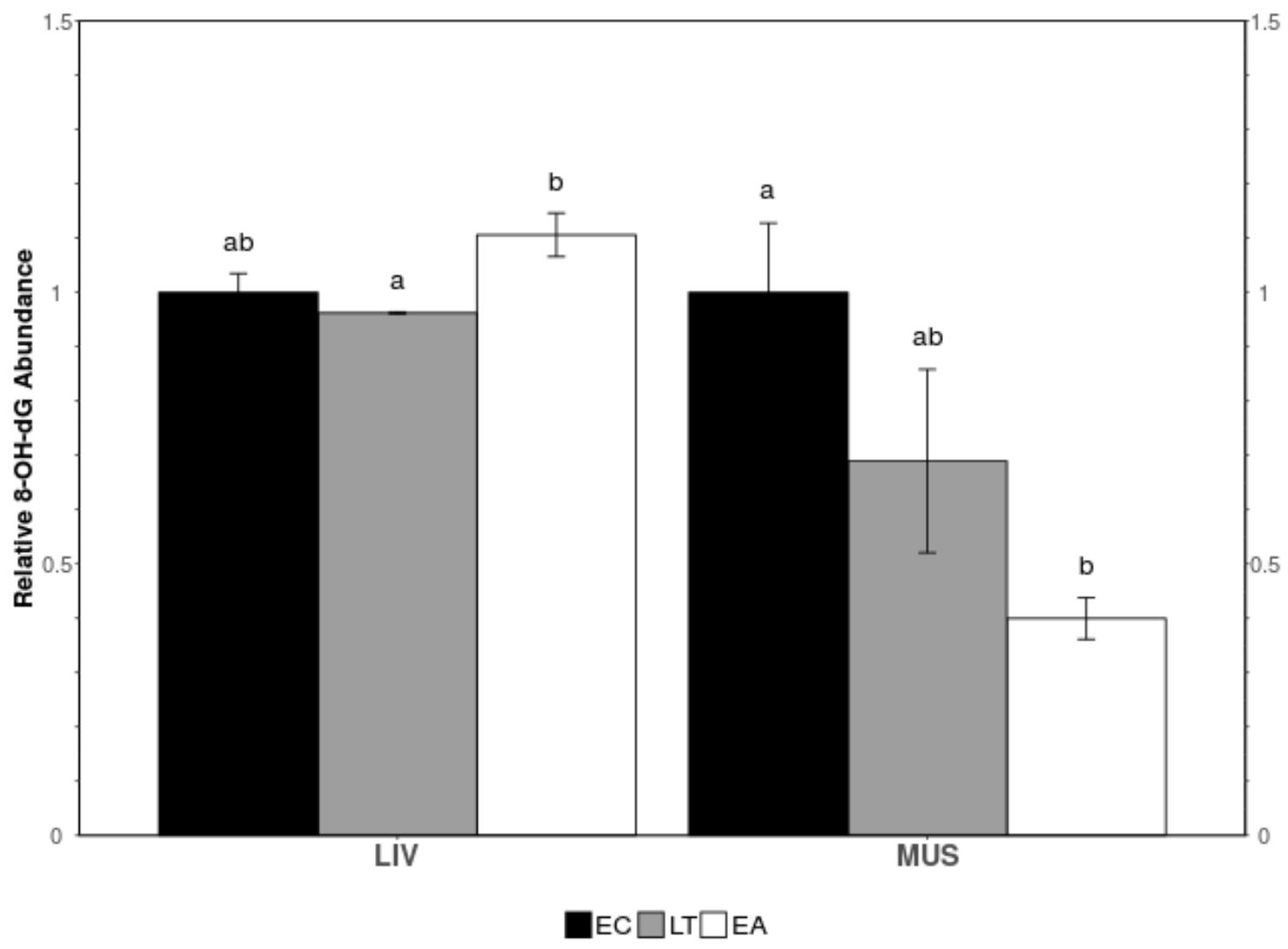

Figure 3.1. Relative abundance of 8-hydroxy-2'-deoxyguanosine in liver and skeletal muscle of Ictidomys tridecemlineatus during late torpor (LT) and early arousal (EA) as compared with euthermic controls (EC). Data is mean \pm S.E.M., $n=4$. Statistical testing used ANOVA plus a Tukey post-hoc test, with $p<0.05$ accepted as a significant difference. Bars sharing the same letter are not significantly different from each other. 


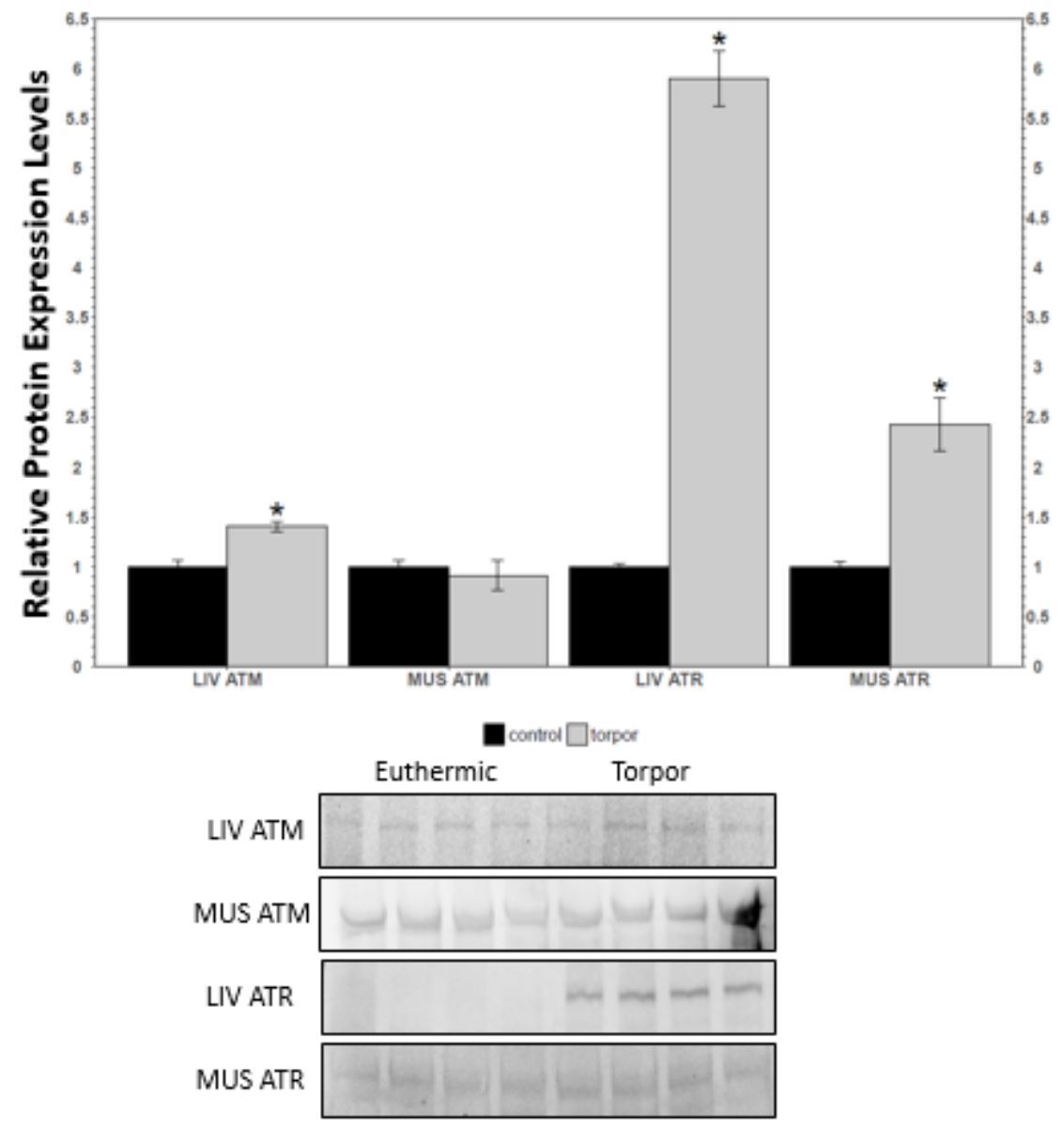

Figure 3.2. Relative protein expression levels of the ATM and ATR kinases in liver and skeletal muscle of Ictidomys tridecemlineatus during torpor. Histograms show mean standardized band densities \pm S.E.M., $\mathrm{n}=4$. Bars marked with an asterisk (*) are significantly different from corresponding euthermic values, $p<0.05$. 

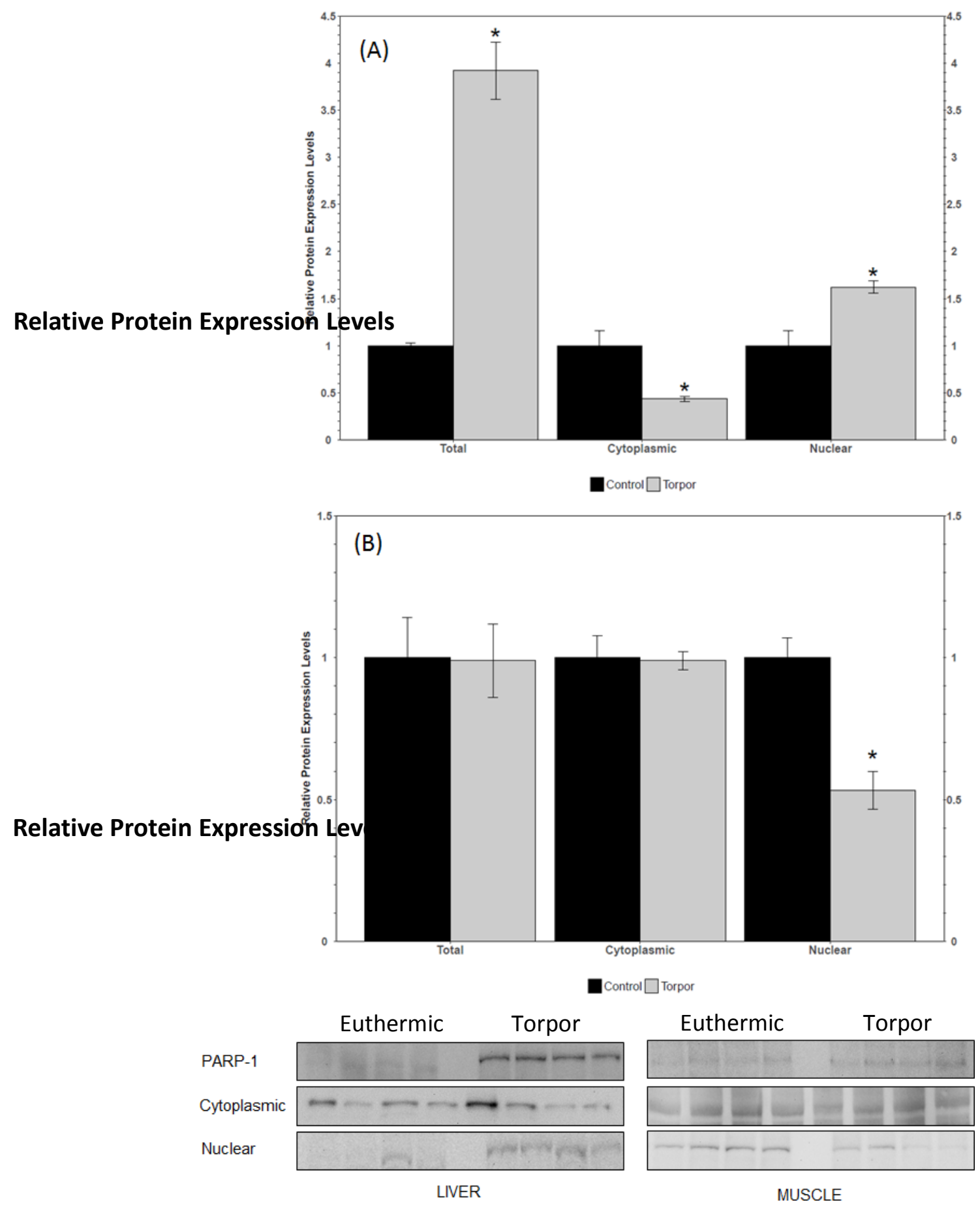

Figure 3.3. Relative protein expression levels of cytoplasmic and nuclear PARP-1 in liver (A) and skeletal muscle (B) of Ictidomys tridecemlineatus during torpor. Histograms show mean standardized band densities \pm S.E.M., $\mathrm{n}=4$. Bars marked with an asterisk $(*)$ are significantly different from corresponding euthermic values, $p<0.05$. 
(A)
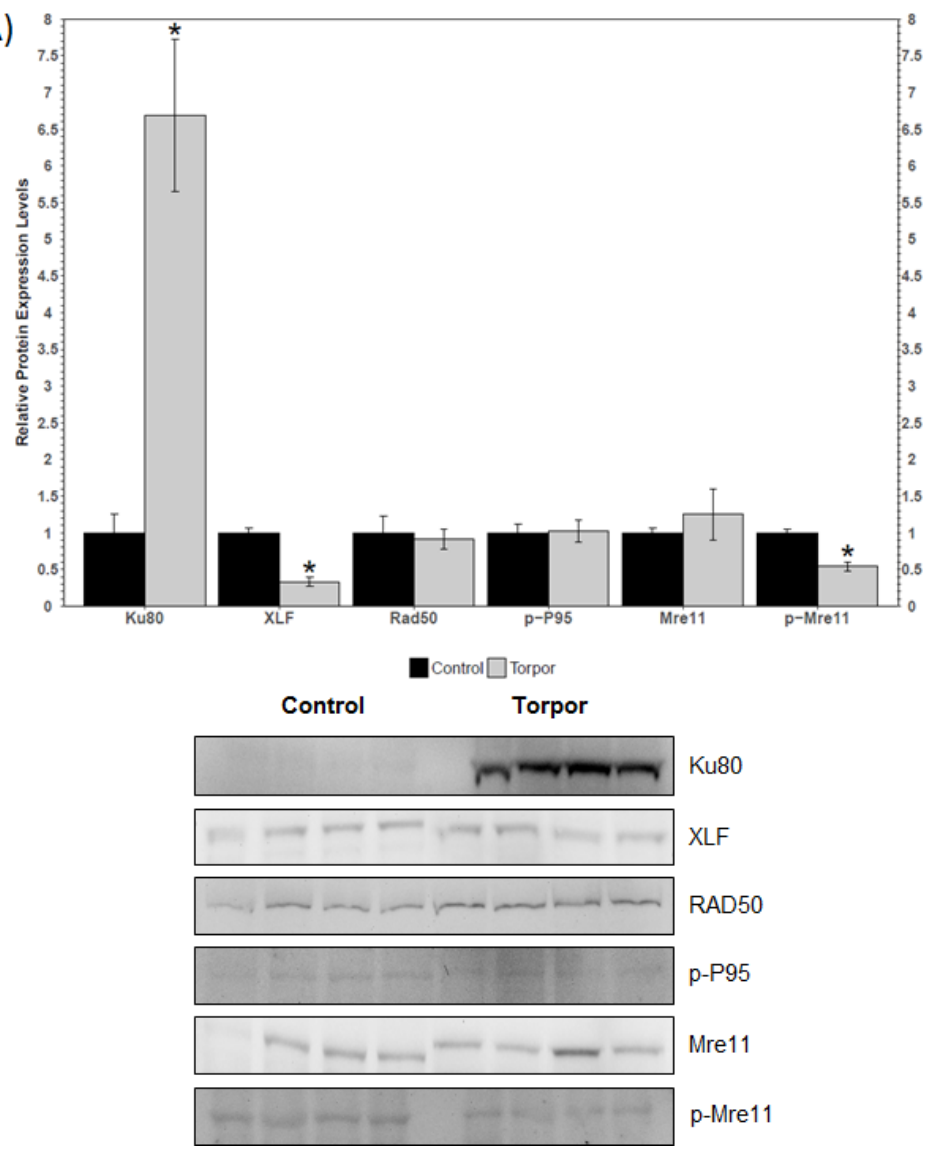

(B)
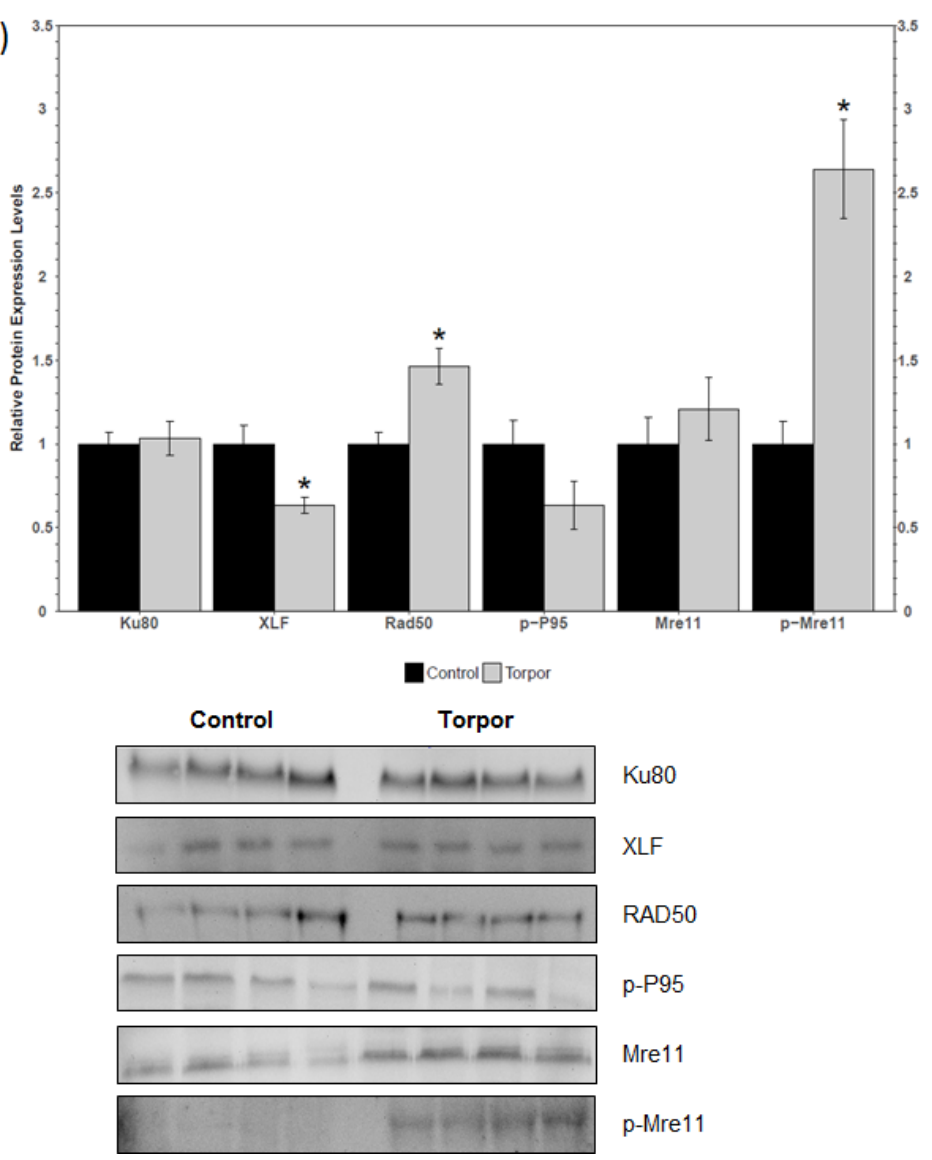

Figure 3.4. Relative protein expression levels of DNA damage repair targets Ku80, XLF, RAD50, p-P95, Mre11, and (total) PARP-1 in liver (A) and skeletal muscle (B) of Ictidomys tridecemlineatus during torpor. Histograms show mean standardized band densities \pm S.E.M., $\mathrm{n}=4$. Bars marked with an asterisk $\left(^{*}\right)$ are significantly different from euthermic values, $p<0.05$. 


\section{CHAPTER 4}

\section{TISSUE-SPECIFIC INTERFERON REGULATION DURING HIBERNATION}




\begin{abstract}
Inflammation has been previously shown to be suppressed during hibernation, but select tissues activate pro-inflammatory pathways, particularly during critical time points during the torpor-arousal cycle. When ground squirrels arouse from torpor, and breathing rates increase, the brown adipose tissue engages thermogenesis to rewarm the body but also appears to induce a strong pro-inflammatory response. Both brown and white adipose tissues have been shown to be key inflammatory organs, both for the recruitment of immune targets, and for the transcription of interferon-regulated genes and induction of signaling pathways. A combination of transcript and protein expression experiments revealed that the adipose tissues induce a strong immune response resulting in the expression of various interferon-related genes especially during the interbout arousal stage of torpor. Brown adipose tissue induced the expression of seven IFN-related genes during interbout arousal and the white adipose tissue induced the expression of four in early arousal and interbout arousal. The results of this study reveal that IRF3 is an important transcription factor which induces the expression of these genes in a tissue-specific and time-specific manner, and is likely a key player in maintaining the "fat but fit" state of the hibernating ground squirrel.
\end{abstract}




\subsection{Introduction}

The thirteen-lined ground squirrel is a champion hibernator that uses an array of molecular adaptations to maintain tissue homeostasis during torpor. Among these are several mechanisms to maintain tissue viability including upregulation of antioxidant defenses $[125,177,178]$ and anti-apoptotic defenses $[45,119,179]$ to prevent cell death. Resulting from the period of hyperphagia in late summer that greatly increases body fat, the switch to a primary reliance on lipid metabolism is made during torpor as well as physiological adjustments including a drop in breathing rate $(<1$ breath/min), heart rate $(5$ 10 beats/min), body temperature $\left(<5^{\circ} \mathrm{C}\right)$ and oxygen perfusion of tissues ( $<10 \%$ of normal). Overall, the hibernating squirrel also drops its metabolic rate by $95-99 \%$ when in full torpor compared to basal euthermic rates $[21,126]$. The immune response has been suggested to be suppressed during hibernation as a means of conserving energy (unpublished work), however, there is current evidence that certain pro-inflammatory targets may be upregulated upon arousal from hibernation to protect from viral pathogens and assist in cell signaling and cytoprotection.

Recent studies have demonstrated that although certain regulators, such as the nuclear factor- $\kappa \mathrm{B}(\mathrm{NF}-\kappa \mathrm{B})$, may be suppressed during torpor (unpublished results), other pro-inflammatory regulators and transcription factors may be preferentially upregulated, in a tissue-specific manner [180] Chronic inflammation is associated with obesity in humans and is a major contributor to insulin resistance, diabetes and other dysfunctional changes in adipose tissues. Even inflammation which develops during periods of overnutrition contributes to a notion of "metainflammation" - a novel type of low-grade, chronic, unresolvable immune response that is in the causal pathway to metabolic dysregulation. This change manifests itself through significant alterations in the number and composition of the immune cell population in various tissues [181]. A significant increase in activated M1 macrophages versus M2 macrophages, elevation of the number of monocytes as well as an increase in the number of cytokines and other inflammatory agents all result in a proinflammatory milieu which can be damaging to surrounding tissues. In previous years, adipose tissue was viewed as an inert tissue whose primary function was the provision of a storage site for triglycerides. However, recent studies have shown that this tissue is an important endocrine organ responsible for the production of 
numerous hormones, cytokines and inflammatory agents which control metabolism, blood pressure, homeostasis and the immune response [47].

The mode of sensing of overnutrition by adipocytes is still unclear, but, a mechanism including members of the Toll-like receptor (TLR) family has been suggested [182]. TLRs are activated by various pathogen-associated molecules and molecular patterns including fatty acids, endotoxin, unmethylated DNA and double stranded RNA. Ligation of broken DNA/RNA ends triggers a signaling cascade which inevitably induces the transcription and the recruitment of various chemokines and cytokines (Kumari et al., 2016). Signaling by TLRs converges on a small set of transcription factors that are believed to mediate the inflammatory transcriptional response. Among these are NF- $\mathrm{B}$, Activated Protein 1 (AP-1) and Interferon Regulatory Factors (IRFs). IRFs comprise a family of 9 members (IRF1-IRF9) and have been implicated in numerous aspects of immune function including macrophage development, $\mathrm{T}$ and $\mathrm{B}$ cell lymphopoiesis and the anti-viral defense [183]. Each IRF contains a well-conserved DNA-binding domain of 120 amino acids, located at the amino terminus and forming a helix-turn-helix motif which recognizes the Interferon (IFN)-stimulated response element (ISRE). Although the IRFs have various immune functions and have distinct roles in the development and function of immune cells (Honda and Taniguchi, 2006), IRF3 has been shown to play a significant role in regulating insulin resistance, acting as a major transcriptional regulator of adipose tissue inflammation and maintaining systemic glucose and energy homeostasis [181].

IRFs have gained much attention as essential regulators of immune cell recruitment with the discovery of pattern-recognition receptors (PRRs) [183, 184]. PRRs recognize pathogen-associated molecular patterns (PAMPs) such as lipopolysaccharide (LPS) and viral nucleic acids. Two classes of PRRs have been identified: the transmembrane PRRs, namely the TLRs, and the cytosolic PRRs, which include the retinoic-acid inducible gene I (RIG-I) family (including melanoma-differentiation-associated gene 5, MDA5). Then, depending on the nature of the pathogen and cell type, these recognition systems are differentially activated, eliciting distinct immune responses [183]. The interaction of dsRNA or dsDNA following infection, triggers an immune response through a cytosolic pattern recognition system. This interaction with the RNA-helicase domain of RIG-I or 
MDA5 induces interactions between the caspase-recruitment domain (CARD) of RIG-I or MDA5 and the CARD-like domain of their adaptor protein mitochondrial antiviral signalling protein (MAVS). This receptor-adaptor interaction results in the activation of TANK (TRAF family member associated NF-KB activator)-binding kinase 1 (TBK1). Activated TBK1 induces phosphorylation of IRF3 (and IRF7), resulting in their homoor heterodimerization and translocation into the nucleus to activate the transcription of IFN genes $[185,186]$.

The current research examines the expression of the IRF3 pathway (Appendix 4.2) and the induction of IFN-regulated genes in the liver, brown adipose and white adipose tissues of the thirteen-lined ground squirrel across the torpor-arousal cycle. To my knowledge, this is the first study of its kind to evaluate the specific mechanisms of IRF3 pathway regulation in the context of metabolic rate depression in a hibernating mammal. Overall, the data suggest a tissue-specific response whereby several genes are induced in the brown adipose during interbout arousal but suppressed or unchanged in the white adipose tissue. Liver data revealed a significant recruitment response for the activation of monocytes across the whole torpor-arousal cycle, but most significantly during early and interbout arousal. 


\subsection{Methods}

\subsubsection{Animal Protocols}

Animal experiments were conducted as previously described in Chapter 2 and [102]. Samples of liver, brown adipose tissue (BAT), and white adipose tissue (WAT), were collected and were flash-frozen in liquid nitrogen, shipped on dry ice to Carleton University, and then stored in $-80^{\circ} \mathrm{C}$ until use.

\subsubsection{Protein quantification}

\section{Total protein Isolation}

Total protein extracts were prepared as described in Chapter 2. Briefly, $50 \mathrm{mg}$ tissue samples were homogenized 1:4 w:v for liver and BAT and 1:3 w:v for WAT in homogenizing buffer $(20 \mathrm{mM}$ Tris base, $150 \mathrm{mM} \mathrm{NaCl}, 1 \mathrm{mM}$ EDTA, $1 \mathrm{mM}$ EGTA, $1 \mathrm{mM} \mathrm{NaF}, \quad 10 \mathrm{mM} \quad \beta$-glycerophosphate, $1 \% \mathrm{v} / \mathrm{v}$ Triton $\mathrm{X}-100$, and $1 \mathrm{mM}$ phenylmethylsulfonyl fluoride). Samples were spun at $10,000 \mathrm{x}$ for 10 minutes at $4^{\circ} \mathrm{C}$ and then supernatants were removed and protein concentrations were determined using the Bio-Rad prepared reagent (BioRad Laboratories, Hercules, CA). All samples were then standardized to a constant $10 \mu \mathrm{g} / \mu \mathrm{L}$ by addition of small aliquots of homogenization buffer, and then aliquots were mixed 1:1 with 2X SDS loading buffer (100 mM Tris base $\mathrm{pH} 6.8,4 \% \mathrm{w} / \mathrm{v}$ SDS, $20 \% \mathrm{v} / \mathrm{v}$ glycerol, $0.2 \% \mathrm{w} / \mathrm{v}$ bromophenol blue, and 10\% v/v 2mercaptoethanol), to yield a final sample concentration of $5 \mu \mathrm{g} / \mu \mathrm{L}$. Finally, samples were boiled for 10 minutes and stored at $-20^{\circ} \mathrm{C}$ until use.

\section{Total Protein Immunoblotting}

Aliquots of 20-30 ug of protein were loaded into wells of 8-12\% SDS-PAGE gels which were run at $180 \mathrm{~V}$ for $45 \mathrm{~min}$. After transfer to PVDF, membranes were blocked using $1-2 \%$ skimmed milk in TBST for $30 \mathrm{~min}$ and were then probed with primary antibodies overnight at $4^{\circ} \mathrm{C}$. Antibodies were all produced in rabbit and were from Cell Signaling: MDA5 Cat\#5321, RIG-I Cat\#3743, MAVS Cat\#3993, TBK1 Cat\#3504, IKKع Cat\# 2905, IRF3 Cat\# 11904, phospho-IRF3 (Ser396) Cat\# 4947, and STING Cat \# 13647. Then, 
membranes were washed and probed with anti-rabbit IgG HRP-linked secondary antibody (diluted 1:5000 in TBST) for $30 \mathrm{~min}$ at room temperature on a rocker.

\section{Quantification and Data Analysis}

Band densities were visualized using enhanced chemiluminescence using a ChemiGenius Bio-Imager (Syngene, Frederick, MD) and quantified by densitometric analysis using GeneTools Software. Band intensities from each lane were standardized against the summed intensity of a group of Coomassie-stained bands in the same lane to correct for variation in sample loading (Eaton, 2013). Mean band densities \pm SEM were calculated and then standardized against the EC (euthermic control) condition. Data are as $\mathrm{n}=4$ independent samples from different animals. All data were analyzed using a one-way ANOVA followed by a Tukey post-hoc test to identify significant differences between each time point. Statistical difference was accepted if $p<0.05$. For each parameter measured, values that were not statistically different from one another share the same letter notation. All statistical analysis and Figure generation was performed using RBioplot [104].

\section{Quantitative PCR}

RNA isolation

RNA isolation was performed as described in Biggar et al., (2018) [187]. Total RNA from each tissue was isolated using Trizol ${ }^{\mathrm{TM}}$ (Invitrogen, Cat\# 15596018). Briefly, $50 \mathrm{mg}$ of tissue was homogenized in $1 \mathrm{~mL}$ Trizol using a Polytron PT10 homogenizer. Next, $200 \mu \mathrm{L}$ of chloroform was added to each sample followed by a brief $30 \mathrm{~min}$ incubation on ice with intermittent vortexing. Samples were centrifuged at $10,000 \times \mathrm{g}$ for 15 min at $4{ }^{\circ} \mathrm{C}$ after which the upper aqueous layer was extracted. RNA was precipitated with $500 \mu \mathrm{L}$ of 2-propanol, followed by a $10 \mathrm{~min}$ incubation at room temperature, and centrifugation as previously described. The supernatant was removed and the pellet was washed with ethanol before centrifugation. RNA pellets were resuspended in $40 \mu \mathrm{L}$ of sterile RNAse-free water. RNA purity was assessed by measuring the $260 \mathrm{~nm} / 280 \mathrm{~nm}$ ratio. RNA integrity was determined by visualizing $18 \mathrm{~S}$ and $26 \mathrm{~S}$ ribosomal bands on a $1 \%$ 
agarose gel with SybrGreen staining. All samples were diluted to $3 \mu \mathrm{g} / \mu \mathrm{L}$ using RNAsefree water.

Following RNA extraction, an aliquot of $171 \mu \mathrm{L}$ autoclaved water was added to each final sample, then 2 -fold serial dilutions of were made $(1 / 2,1 / 4,1 / 8,1 / 16,1 / 32)$. Forward and reverse primers for each downstream interferon gene were designed based on the sequence data from NCBI data bank and were synthesized by Sigma Genosys. Primer sequences can be found in appendix 4.1. Tissue-specific housekeeping genes were used as controls; BAT - tbp; LIV - $\alpha$-tubulin; and WAT - $\alpha$-actin; Amplification was performed as described previously [188] with an annealing temperature of $57^{\circ} \mathrm{C}$. Critical threshold $(\mathrm{Ct})$ values for each animal condition (EC, EN, LT, EA, and IA) were standardized to the $\mathrm{Ct}$ of the respective housekeeping gene from the same sample. The statistical comparative threshold cycle $\left(\Delta \Delta \mathrm{C}_{\mathrm{t}}\right)$ method was used to determine relative gene expression for each tissue. Data are expressed as relative mean expression ( \pm SEM) for $n=4$ independent samples from different animals. Statistical analysis of the data was performed using a oneway ANOVA followed by a post-hoc Tukey test to identify significant differences among groups. Statistical difference was accepted if $p<0.05$. For each parameter measured, values that were not statistically different from one another share the same letter notation. All statistical analysis and Figure generation was performed using RBioplot [104]. 


\subsection{Results}

\subsubsection{IRF3 transcript levels}

The IRF3 transcription factor is responsible for inducing the expression of many immune genes including interferon-regulated genes, cytokines, interleukins and other inflammatory targets. Figure 4.1 shows the transcript levels of IRF3 in liver, BAT and WAT across all timepoints. Data for liver revealed a decrease in protein expression in late torpor to $40 \pm 6 \%$ of control values followed by a gradual return to control values during arousal. Conversely, brown adipose tissue showed no significant change in IRF3 transcripts until the interbout arousal timepoint when protein levels decreased to $37 \pm 8 \%$ of control values. However, interestingly, white adipose tissue showed a significant increase to over 3 -fold $(3.09 \pm 8.8 \%)$ of control values during interbout arousal.

\subsubsection{Protein Expression changes}

Changes in IRF3 protein and phospho-protein expression across the torpor-arousal cycle in ground squirrel liver are shown in Figure 4.2. Total IRF3 protein levels increased significantly by nearly 2 -fold $(1.89 \pm 0.04)$ during entrance into hibernation, followed by a return to control levels in torpor and early arousal. Subsequently levels dropped strongly during interbout arousal to a value just $18 \pm 3 \%$ of control levels. Phosphorylated IRF3 levels were maintained at control values during entrance into torpor (EN) and long-term torpor (LT) but then fell by about one-half during in early arousal $(53 \pm 5 \%)$ and remained low in interbout arousal $(55 \pm 3 \%)$, as compared with ACR.

Figure 4.3 reveals the relative changes in the liver of upstream RIG-I pathway proteins. Inhibitor of nuclear factor kappa-B kinase subunit epsilon (IKKe), one of the activators of IRF3, showed a significant decrease in protein expression during entrance into torpor to $57 \pm 4 \%$ of control values and this was maintained through late torpor and early arousal (52 $\pm 7 \%$ and $67 \pm 3 \%$, of EC values, respectively), followed by a further decrease during interbout arousal to just $27 \pm 9 \%$ of EC values. The MAVS protein showed a significant decrease during entrance into torpor to $42 \pm 8 \%$ of control values, but returned to control levels during late torpor and increased significantly during early arousal and interbout arousal to $1.38 \pm 0.10$ and $1.48 \pm 0.01$-fold, respectively, over EC values. MDA5, 
like RIG-I, is a cytosolic RNA helicase which functions as a pattern recognition receptor typically for viral sensing. MDA5 protein expression levels were generally unchanged across the torpor-arousal cycle aside from a decrease during late torpor to $44 \pm 4 \%$ of control values followed by a return to control levels in IA. Expression levels of TBK1, another activator of IRF3, were also unchanged compared to EC values except for a significant decrease during early arousal to $76 \pm 3 \%$ of EC and that was significantly decreased in comparison to late torpor which was $1.3 \pm 0.13$ fold higher than control levels. RIG-I levels decreased strongly during late torpor to $52 \pm 7 \%$ of EC but returned to control values in EA before dropping again to $37 \pm 2 \%$ of EC values during interbout arousal. Finally, STING levels were generally unchanged in comparison to controls, but revealed a significant increase during early arousal in comparison to entrance to $1.15 \pm 0.11$-fold than EC values, followed by a further increase to $1.44 \pm 0.12$-fold higher than EC during interbout arousal. The overall trend seen in the liver points to a maintenance of protein expression during crucial stages of torpor such as during entrance or late torpor followed by either a return to control levels or an increase in expression compared to control levels during the stages of arousal including early arousal and interbout arousal.

The brown adipose tissue revealed a different trend with regard to IRF-3 and its upstream targets whereby very few targets were seen to be suppressed. Interestingly, total IRF-3 levels increased during late torpor and early arousal to nearly 2 -fold higher than control values at $1.99 \pm 0.12$ and $1.98 \pm 0.24$-fold over EC, respectively (Figure 4.4). By contrast, phosphorylated IRF-3 levels did not correspond to total levels as a small drop in expression was seen during entrance to $68 \pm 5 \%$ of EC values, followed by a return to EC levels for the remainder of the timecourse. Many RIG-I pathway proteins in brown adipose were maintained or even induced across the torpor-arousal cycle. With respect to upstream IRF3 activators (Figure 4.5), the two kinases IKK $\varepsilon$ and TBK1 revealed different trends whereby IKK $\varepsilon$ showed a maintenance of protein expression during entrance and late torpor to levels that were not significantly different than $\mathrm{EC}$ values but then dropped during early arousal and interbout arousal to levels that were $69 \pm 9 \%$ to $64 \pm 2 \%$ of control values. Conversely, TBK1 was seen to increase significantly in protein expression during late torpor and early arousal to $1.71 \pm 0.01$ and $1.51 \pm 0.07$-fold of control values, followed by a return to control values, but which were significantly decreased compared to entrance at 
$75 \pm 2 \%$ of control values. The two helicases, MDA5 and RIG-I both showed no significant changes across the torpor-arousal cycle, aside from a significant increase in MDA5 during early arousal to $2.21 \pm 0.19$-fold of control values. Finally, STING protein expression levels were elevated during late torpor to $1.29 \pm 0.09$-fold higher than control values followed by a drop again in EA then a stronger increase to $1.72 \pm 0.06$-fold higher than EC values during interbout arousal. MAVS revealed no significant change throughout the cycle.

The white adipose tissue showed the strongest response for induction of the RIG-I pathway whereby both total and phosphorylated IRF-3 protein expression levels were found to increase strongly but at varying points of the torpor-arousal cycle (Figure 4.6). Total IRF3 protein expression levels rose to nearly 4-fold (3.92 \pm 0.47$)$ during late torpor, and remained elevated for the rest of the time course at $3.14 \pm 0.38$-fold higher than EC levels in early arousal and $2.11 \pm 0.40$-fold higher than controls during interbout arousal. However, phosphorylated IRF3 levels were highest during entrance into torpor at $2.5 \pm$ 0.15 -fold above controls followed by a drop to $56 \pm 12 \%$ of control levels during late torpor. Then, during early arousal and interbout arousal, levels returned to values not different from the EC controls. Figure 4.7 reveals the expression levels of upstream regulators of IRF3 in white adipose, showing very strong upregulation of three targets. IKK $\varepsilon$ kinase protein expression levels were significantly induced during late torpor and early arousal at $4.78 \pm 0.22$-fold and $4.13 \pm 1.01$-fold, respectively, over EC values followed by a return to control values during interbout arousal. MAVS was also induced during late torpor to $3.44 \pm 0.76$-fold over control values and MDA5 was elevated in late torpor through to interbout arousal at $4.83 \pm 0.15$-fold, $5.09 \pm 0.57$-fold and $4.85 \pm 0.72$ fold over EC values, respectively. TBK1, RIG-I and STING expression levels were all maintained throughout the torpor-arousal cycle aside from a decrease in RIG-I during late torpor through to interbout arousal to $61 \pm 4 \%, 42 \pm 2 \%$, and $53 \pm 5 \%$, respectively, as compared with EC.

\subsubsection{Downstream transcript level changes}

Next, the transcript levels of downstream IRF3-regulated genes were assessed to determine whether changes in IRF3 levels resulted in altered transcripts levels of 
downstream targets. Figure 4.8 shows the transcript expression levels of six downstream genes regulated by IRF3 in liver. CXCL10 transcripts showed a downward trend in late torpor followed by a significant increase during early arousal to $1.42 \pm 0.30$-fold higher than control values which was significant compared to late torpor but not control values. IFN- $\beta$ values showed no significant change across the torpor-arousal cycle, as did the IFNReceptor, however IFIT2 levels rose significantly during late torpor and interbout arousal to $1.61 \pm 0.12$-fold and $1.54 \pm 0.15$-fold, respectively over EC values. Similarly, transcript levels of GPB-1-Like gene revealed a significant increase at two time points, entrance and early arousal, to $3.51 \pm 0.69$-fold and $3.03 \pm 0.63$-fold over control values, respectively. Finally, transcript levels of the cytokine CCL5 showed the most pronounced changes whereby a significant increase was seen during entrance into torpor to $3.15 \pm 0.10$-fold of control values, followed by an even stronger increase during early arousal to $5.20 \pm 0.26$ fold that remained elevated during interbout arousal at $5.06 \pm 0.51$-fold over EC values.

Brown adipose tissue also showed differential expression of IRF3 downstream genes, as well as in some additional adipose-specific genes examined (Figure 4.9). Two of the IFITs (IFIT1 and IFIT2) showed similar expression patterns whereby there was little charge across most of the torpor-arousal cycle but a strong increase during interbout arousal; levels of IFIT1 in IA rose to $3.92 \pm 0.45$-fold over EC values whereas IFIT2 increased to $1.48 \pm 0.04$-fold over control values during interbout arousal. However, IFIT3, CCL2, and CCL5 transcript levels showed no significant change across the torpor-arousal cycle. IFN $\alpha$-2-like transcript levels showed a small increase during late torpor, but were very strongly increased during interbout arousal to values that were $4.70 \pm 0.30$-fold over EC. IFN $\beta$ transcript levels showed a similar pattern with a significant increase during late torpor to $2.06 \pm 0.36$-fold of control values followed by a rise to $5.63 \pm 0.20$-fold over EC values. The IFN-receptor showed no significant change throughout the cycle aside from a significant increase during interbout arousal to $3.88 \pm 0.20$-fold of control values. The two adipose-specific transcripts OASL2 and RSAD2 both revealed very similar results. OASL2 transcript levels decreased during entrance to $48 \pm 7 \%$ of control values, followed by a return to control levels, and a significant increase during interbout arousal to $2.74 \pm 0.09$ fold of EC values. Similarly, RSAD2 transcript levels decreased during entrance to $54 \pm$ 
$8 \%$ of control values, followed by a return to control levels, and a similar increase during interbout arousal to $1.45 \pm 0.05$-fold of control values.

Finally, white adipose tissue transcript levels were examined and revealed different expression patterns than the brown adipose tissue or liver (Figure 4.10). IFIT2, CCL5, and RSAD2 showed no significant changes in expression across the torpor-arousal cycle, but the remaining targets revealed differential changes. IFIT1 showed no significant changes during entrance into hibernation, but a decrease was noted during late torpor to $34 \pm 5 \%$ of EC values. Although not significantly different compared to control or entrance values, it

was significantly different compared to the elevated IFIT1 values noted during early arousal and interbout arousal $(1.90 \pm 0.35$-fold and 1.81 \pm 0.29 -fold, higher than EC respectively). IFIT3 transcript levels were suppressed during entrance and remained low throughout all subsequent stages with values, ranging from $56 \pm 7 \%$ (EN) down to $34 \pm$ $7 \%$ (EA) of the EC values. CCL2 showed a very similar pattern whereby a significant decrease in transcript levels was observed during entrance $(49 \pm 9 \%)$ that was maintained through to early arousal $(33 \pm 4 \%)$ as compared with EC but followed by a rise again interbout arousal. Relative transcript levels for IFN $\alpha-2-\mathrm{L}$ were not significantly different from EC during EN, LT or EA stages but showed a very strong increase during interbout arousal to $6.73 \pm 2.07$-fold of control values. IFN $\beta$ and the IFN receptors revealed similar patterns of expression whereby no change was seen until interbout arousal when a $3.61 \pm$ 0.44 -fold increase in IFN $\beta$ was noted and a $1.54 \pm 0.10$-fold increase in IFNR. OASL2 revealed a significant decrease during late torpor to $77 \pm 15 \%$ of control values, however this was followed by a return to control levels for the rest of the cycle.

\subsection{Discussion}

It has been long suggested that the inflammatory response is suppressed during hibernation as part of an effort to reduce energetic demands during torpor. This has been supported in several studies, including the classical example of the little brown bat, Myotis lucifugus, which succumbs to infection from the Pseudogeomyces destructans fungus, also known as "white nose syndrome" as a result of reduced immune defenses during hibernation [189]. This fungus appears on the wings and nose of bats during their 
hibernation, enters the nasal cavity and infects their airways, during a this time of suppressed metabolism, immune response, and wound healing [190]. According to previous research, a local inflammatory response is initiated whereby the expression of cytokines, interleukins and several chemokines is induced, however immune cells such as neutrophils, leukocytes and T cells do not appear to be recruited [189]. However, the current study on ground squirrels reveals that both protein and transcript expression levels of several immune targets are, in fact, maintained at control levels or even increased over the torpor-arousal cycle, in a tissue-specific manner. Some potential explanations for the difference between bats and ground squirrels could be that perhaps the little brown bat does not exhibit the same level of control over its immune system as the ground squirrel, or that the fungus simply overwhelms the bat's suppressed immune system, or perhaps the fungus was simply better able to evade detection and was therefore able to overtake the bat's immune system before it could surmount a response. As well, the bat does not arouse during the hibernation period whereas the squirrel undergoes multiple bouts of torpor arousal, and could therefore be giving the squirrel a chance to activate its immune system. And finally, the proximity of bats to one another during hibernation is more likely to promote the spread of disease or infection, whereas squirrels generally hibernate in solitude in cold dens.

The present study focused on antiviral immune defenses of ground squirrels. For any organism to assemble a successful antiviral defense, the upregulation of type-I IFN genes, enabled by the concerted activation of several transcription factors (most notably

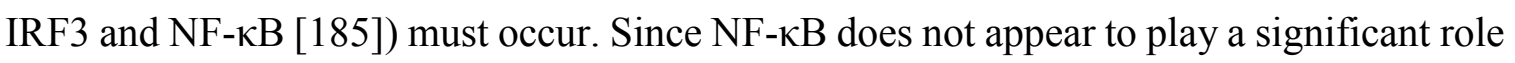
during the torpor-arousal cycle, I chose to examine the role of IRF3 in mediating the immune response. IRF3 transcript levels revealed differential expression changes among the three tissues examined (liver, BAT, WAT) (Fig 4.1) where the greatest response was noted in WAT. These results for mRNA transcript levels did not correlate directly with changes in IRF3 protein levels but this can be expected since processing of gene transcripts can be affected in multiple ways including via post-translational modifications, the action of microRNAs, transcript degradation or shunting into storage in stress granules or pbodies [18]. 
In the liver, total IRF protein levels showed a peak during the entrance phase and decreased strongly during interbout arousal, but expression levels of the active phosphorylated form were much less affected, showing a drop during arousal only (Fig 4.2). This suggests that IRF3 is phosphorylated and possibly translocated into the nucleus for the activation of IRF3-dependent genes during the preparatory stages of torpor and torpor itself, but its functions are not required during the arousal stages. Expression levels of TBK1, the direct upstream activator of IRF3, appear to correlate with the results for IRF3 and were maintained throughout the cycle aside from a decrease during early arousal. The overall increase could suggest IRF3 activation and phosphorylation during those times (Fig 4.3). Another activator of IRF3 is the Stimulator of IFN Genes (STING) that was found to be induced during interbout arousal, suggesting another mechanism of IRF3 activation. STING activates IRF3 via TBK1 by functioning as a scaffold protein to IRF3 and TBK1 and selectively promotes IRF3 phosphorylation [191, 192]. The two PRRs, MDA5 and RIG-I, both revealed a maintenance of expression during entrance, but were both significantly reduced during torpor, followed by an induction during arousal. As sensors of foreign material, it would appear that their expression is, in fact, reduced during the hypometabolic period but is turned on again during arousal. This supports previous studies which showed that inflammation was suppressed in hibernation, possibly as a means of conserving energy. Downstream of RIG-I, the MAVS protein functions to recruit IKK $\varepsilon$, and TBK1. MAVS expression was reduced during entrance but was then returned to control levels during late torpor and was then induced during the arousal stage. This could suggest that there is an induction in the interaction between MAVS and RIG-I during the arousal stages of the cycle. IKK $\varepsilon$ and TBK1 then function to activate and phosphorylate IRF3. IKK $\varepsilon$ was found to be significantly suppressed during all stages of the cycle, perhaps suggesting a rate-limiting step for IRF3 activation. Due to its proinflammatory function, IKK $\varepsilon$ activity is tightly controlled. One study identified two isoforms of IKK $\varepsilon$ that originate from alternative splicing and have the potential to inhibit the activity of the full-length protein [185]. Additionally, a splice variant of the IKKe-related kinase TBK1 (TBK1s) also negatively regulates virus-triggered type-I IFN expression and could be responsible for restraining or turning off the antiviral signaling pathway as it is specifically upregulated after viral infection [193]. TBK1s inhibits RIG-I, but not TBK1-mediated activation of 
ISRE and the IFN- $\beta$ promoter. It also negatively regulates the virus-triggered IFN- $\beta$ signaling pathway by disrupting the interaction of RIG-I with MAVS and negatively regulates virus-triggered IRF3, but not NF- $\mathrm{kB}$ [193]. TBK1s is similar to MyD88, a splice variant of MyD88 which differentially modulates NF- $\kappa B$ and AP-1-dependent gene expression of the TLR signaling pathway [194]. Furthermore, MAVS selectively suppressed retinoic acid-inducible protein I-mediated NF- $\mathrm{kB}$ activation but enhanced the activity of the IFN- $\beta$ promoter, and TAG, a splice variant of TRAM, inhibited only activation of IRF3 but not NF- $\kappa$ B. Therefore, alternative splicing represents an effective, yet complex regulatory mechanism that the ground squirrel may likely employ to fine-tune the immune response during the hibernation period [185].

Similarly, in the brown adipose tissue, there appeared to be an overall maintenance of phosphorylated IRF3 expression (Fig 4.4), which was generally observed in the upstream activators as well (Fig 4.5). The two upstream sensors, MDA5 and RIG-I were elevated during early arousal (although RIG-I was not a significant change) as well as several other targets. This supports the idea that IRF3 activation in BAT was generally maintained at control levels throughout the torpor-arousal cycle but was induced during early arousal, potentially to fight off pathogens that might become active as body temperature rose back to euthermic $\left(37^{\circ} \mathrm{C}\right)$ values.

Finally, white adipose tissue showed a strong increase in total IRF3 levels during torpor and early arousal but phosphorylated IRF3 expression levels revealed a significant increase only during entrance (Fig 4.6). Expression levels of downstream targets in WAT revealed a strong increase in several targets including IKK $\varepsilon$, MAVS and MDA5 during the late torpor, early arousal, and in the case of MDA5, also into interbout arousal (Fig 4.7). The other targets in WAT were largely across the torpor-arousal cycle, aside from a decrease in expression in RIG-I from late torpor onwards. This could suggest that there could be a different sensing mechanism for RIG-I and MDA5. In fact, although RIG-I and MDA5 both act as sensors of dsDNA in the cytoplasm and initiate an interferon-dependent inflammatory response, they do still have distinct but complementary ligands and signaling mechanisms. RIG-I sequesters DNA by the Caspase Activation and Recruitment Domain (CARD), but not by MDA5 in the absence of a ligand. MDA5 also has a much greater 
propensity to form filaments along dsDNA, and the contribution of K63-linked ubiquitin chains is different for both, but is far less defined for MDA5. Therefore, it is plausible that an increase in one PRR could result, and not the other [195]. Similarly for IKK $\varepsilon$ and TBK1, the lack of change in TBK1 could suggest a layer of regulation for the activation of IRF3. Overall, it would appear that there could be a specific induction in IRF3 activation in white adipose tissue, dependent on MDA5 signaling.

In order to examine the expression of downstream IFN-related genes, the mRNA transcript levels of various targets were measured. In the liver, several IFN-related genes, cytokines and antiviral proteins were found to be differentially regulated (Fig 4.8). CXCL10, a cytokine responsible for leukocyte trafficking, was significantly upregulated during early arousal, as compared to late torpor. IFIT2, an antiviral protein which inhibits the expression of viral messenger RNAs was found to be upregulated during late torpor and interbout arousal. GBP-1-L, a protein responsible for exhibiting antiviral activity against influenza, promoting oxidative killing and delivering antimicrobial peptides to autophagolysozomes, was also significantly induced during entrance, and early arousal. And finally, CCL5, a cytokine responsible for recruiting monocytes to sites of infection, was significantly increased at almost all time points across the cycle, as compared with EN. This suggests that the liver is still activating IFN-related genes but generally at critical time points of the cycle - namely during entrance and early arousal.

In the brown adipose tissue, however, an interesting pattern emerged. Many of the targets examined were maintained at control levels across most of the cycle, but were then significantly and strongly upregulated during interbout arousal (Fig 4.9). IFIT1, IFIT2, IFN $\alpha-2-L, I F N \beta$, IFNR, OASL2 and RSAD2 were all upregulated during this time. The 2'5'-oligoadenylate synthase-like protein 2 (OASL2) and Radical SAM Domain-Containing 2 (RSAD2) protein are two interferon-induced inflammatory targets that have been shown to play a significant role in adipose inflammation [181, 196, 197]. This is in contrast to white adipose tissue which showed suppression of expression for several targets or no change across the cycle (Fig 4.10). Only IFIT1, IFN $\alpha-2-L$, IFN $\beta$ and IFNR were found to be induced during early arousal and/or interbout arousal. It would appear that BAT is inducing the expression of interferon-regulated genes. The interferon receptor and the two 
proteins $(\alpha$ and $\beta)$ are responsible for the innate immune response and are produced by fibroblasts, dendritic cells and monocytes [198]. They function to protect against viral infection but also have numerous other functions including cellular communication, signal transduction, as well as regulating growth and differentiation, and can be produced in high abundance in a short period of time in response to stress or toxicity [199]. Therefore, BAT may be maintaining the levels of these immune factors and then increasing them during arousal as a cytoprotective mechanism.

It is important to note, however, that the induction of interferon-related genes is not only present in response to viral infection. Numerous studies have shown an induction in the expression of these genes in response to other proinflammatory states such as during metabolic and hepatic diseases characterized by overnutrition [200-203]. Although ground squirrels are known to accumulate large amounts of fat prior to winter hibernation, these animals are "fat but fit" and do not accrue the damage associated with obesity-related metabolic diseases. In a disease or obese state, the adipose tissue can account for over $50 \%$ of body mass and exist in various depots including omental, subcutaneous, mesenteric, brown adipose and visceral sites surrounding the organs [48]. Adipose tissue contains varying types of immune cells including macrophages, $\mathrm{T}$ cells, eosinophils, lymphocytes and many others which are present at high concentrations, especially in obese states. These cells produce immune factors that have been shown to modulate adipocyte function and regulate the inflammatory environment in a dynamic tissue that changes in response to daily feeding and fasting. These effects can be seen as early as 2-3 days after switching to a high-fat diet in mice [204] and TLR activation through fatty acid sensing is one of the proposed mechanisms for these pathways and is likely dysregulated in disease states.

In conclusion, ground squirrels appear to regulate immune pathways in such a way that the concentration of immune cells is not elevated, even in response to high-fat feeding. They are able to suppress the pro-inflammatory response, until such time that it is required, and regulate it in a highly controlled, tissue-specific manner. The liver maintains basal levels of immune function, but brown adipose initiates a strong immune response induced by the expression of interferon-regulated genes. In this way, the ground squirrel is able to 
protect itself from foreign pathogens and viruses, as well as maintain cytoprotection and activate numerous signaling pathways over the course of its winter hibernation. 


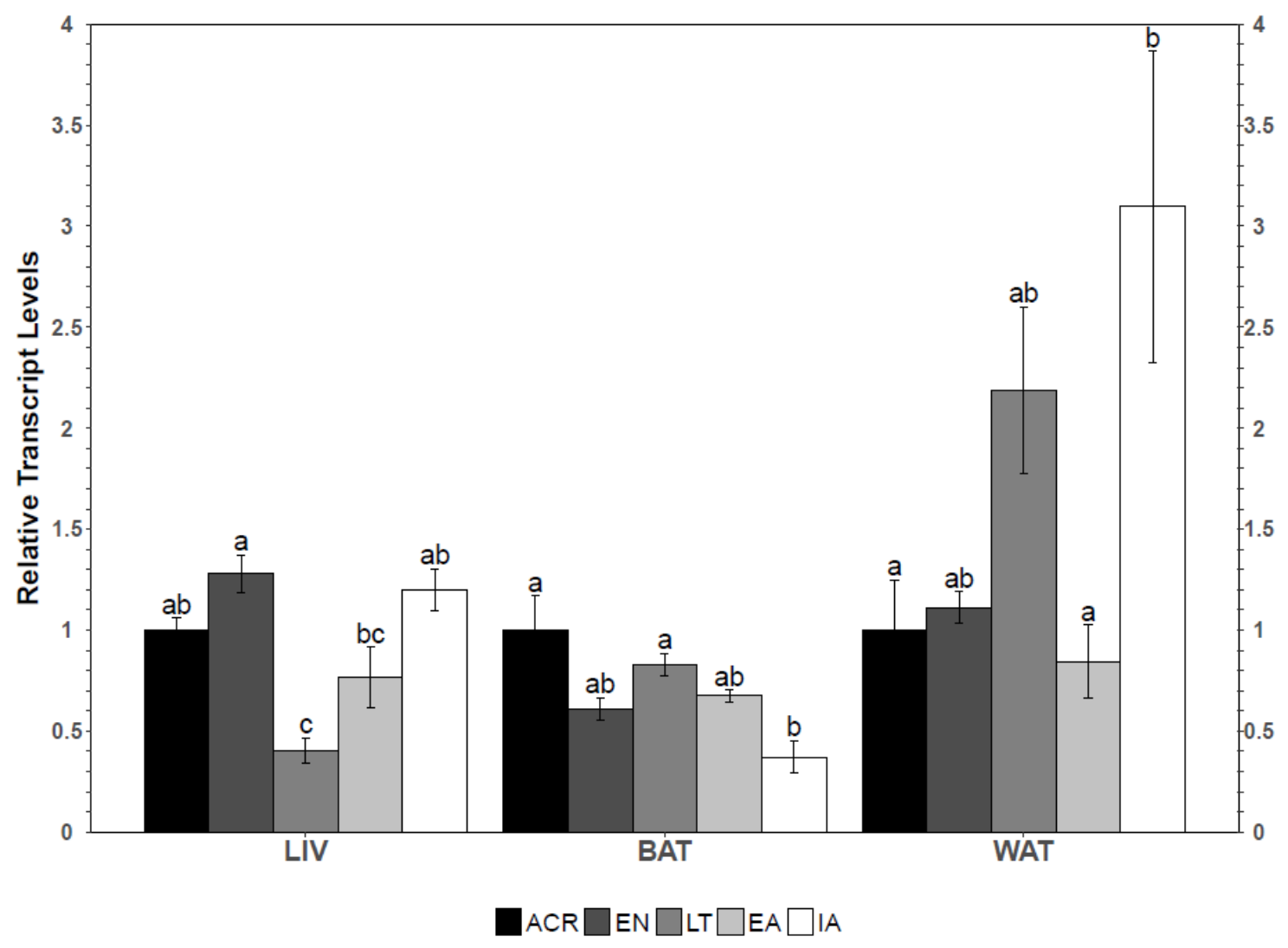

Figure 4.1: Histogram of normalized $I R F 3$ relative gene transcript levels across the torporarousal cycle in liver (LIV), brown adipose tissue (BAT) and white adipose tissue (WAT). Data are mean critical threshold (Ct) $( \pm$ S.E.M. $n=4$ independent trials on tissue from different animals). Values that share the same letter are not significantly different ( $p<$ $0.05)$. 


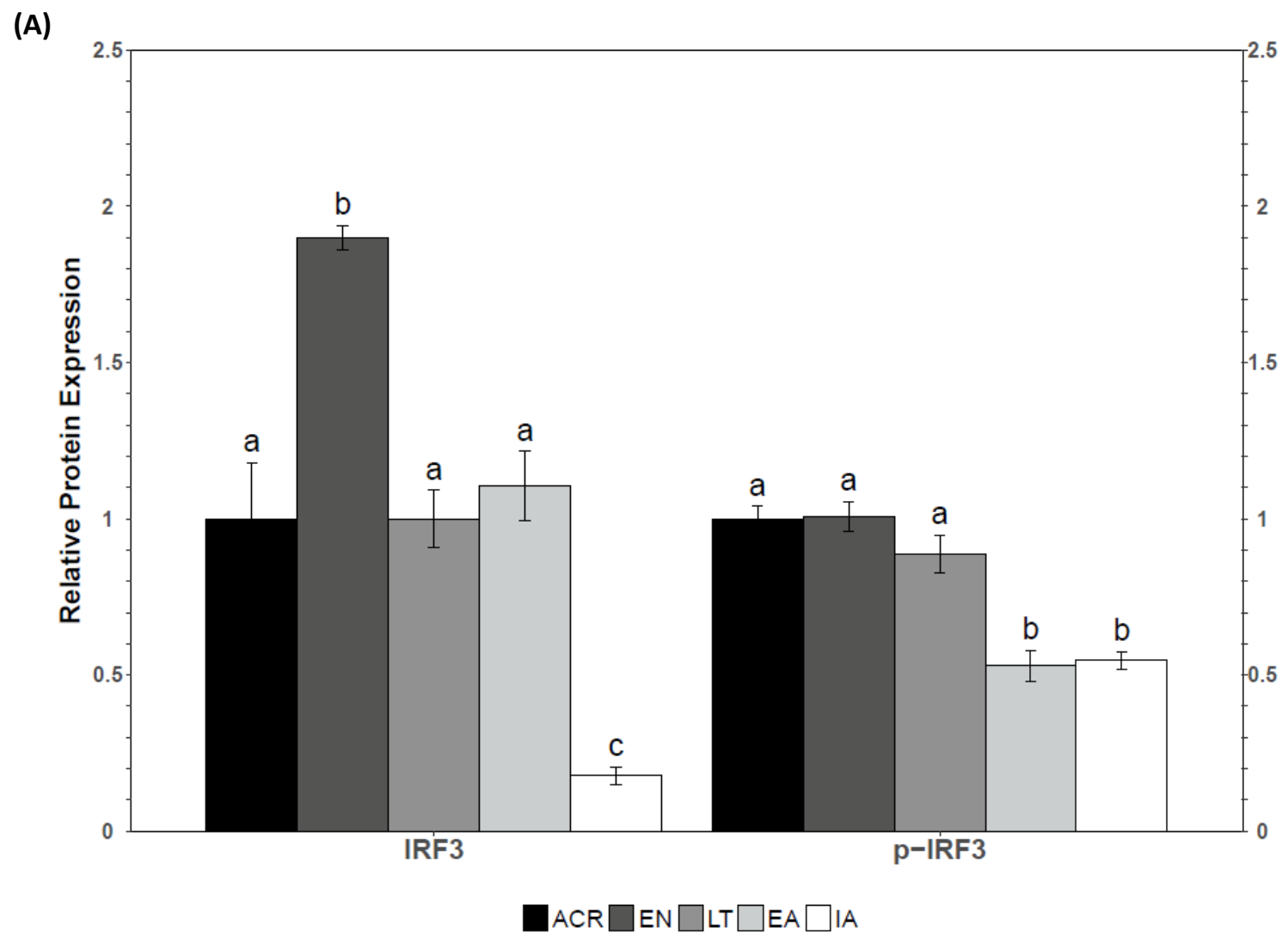

(B)

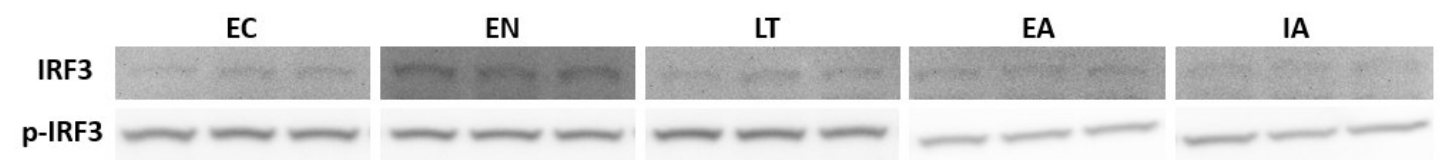

Figure 4.2: Relative protein expression levels of total IRF3 and phosphorylated IRF (Ser396) in Ictidomys tridecemlineatus liver tissue across the torpor-arousal cycle. (A) Histogram showing mean standardized expression levels of IRF3 and p-IRF3 ( \pm S.E.M., $n$ $=4$ independent protein isolations from different animals). (B) Representative western immunoblot bands illustrating the expression levels of IRF3 and p-IRF3. Data were analyzed using a one-way ANOVA followed by a Tukey post-hoc test $(p<0.05)$. For each parameter measured, values sharing the same letter notation are not statistically different from one another. 
(A)

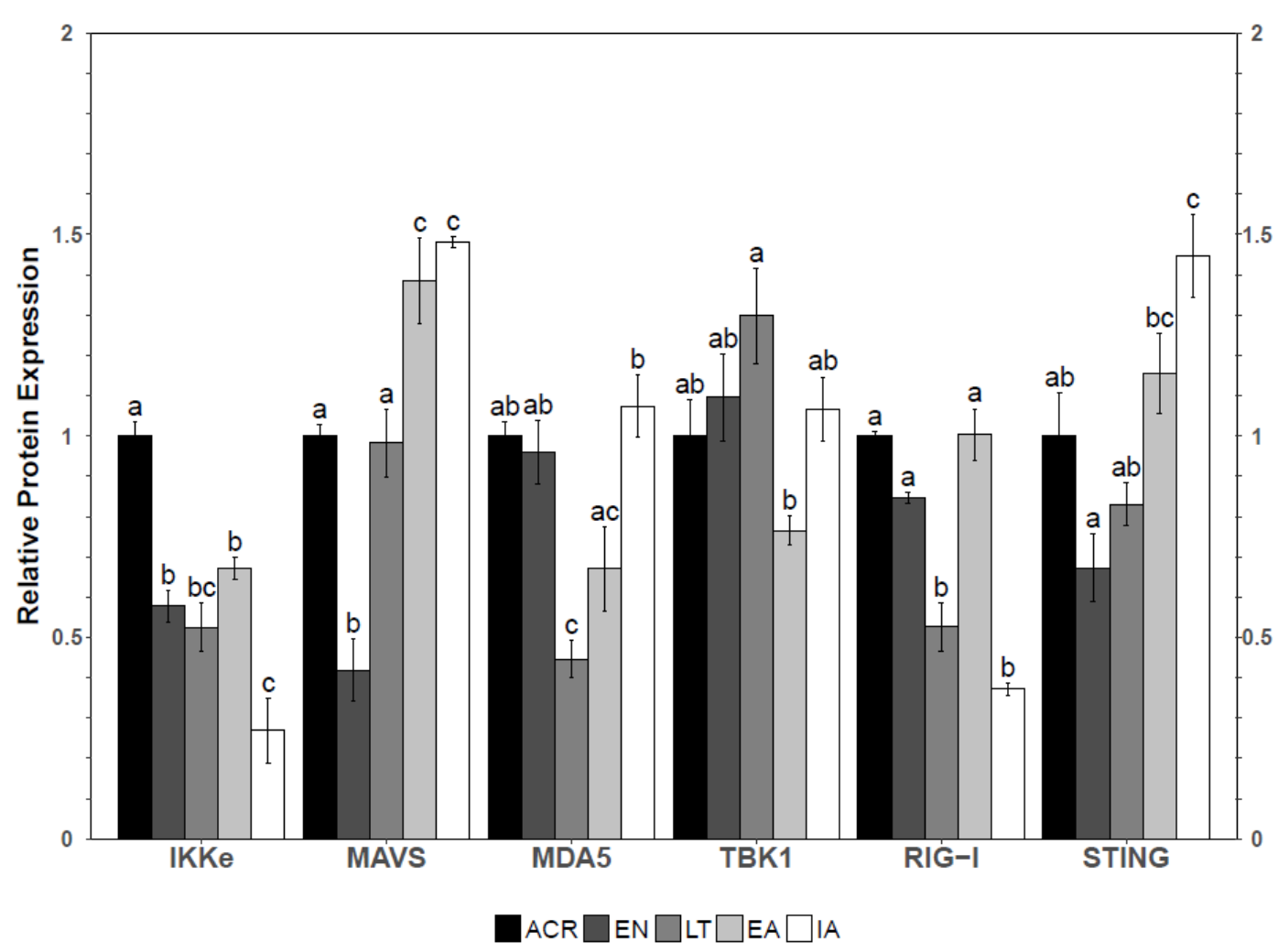

(B)

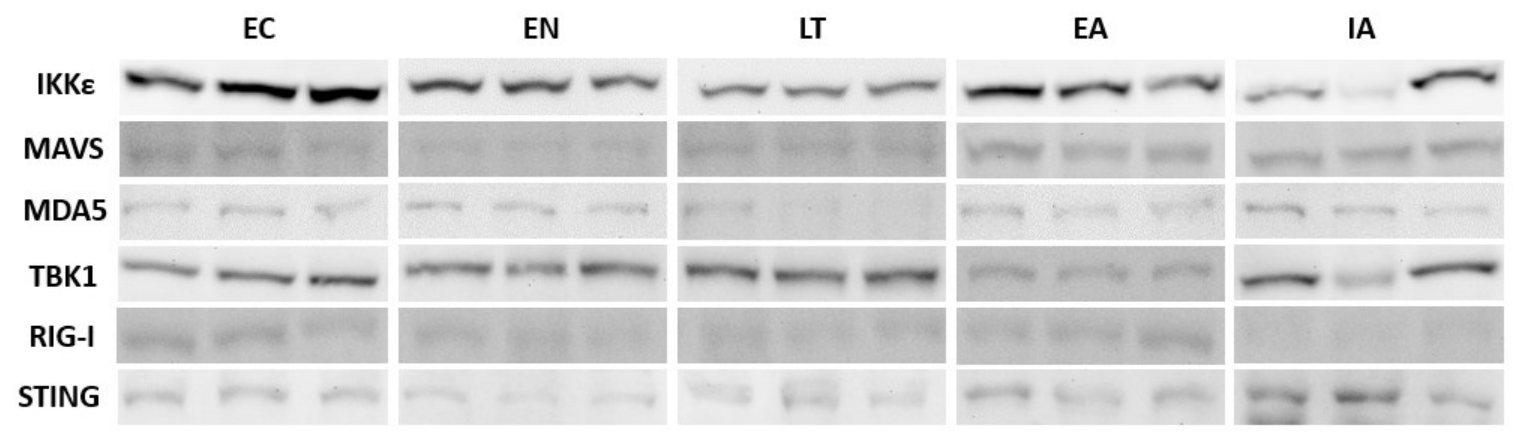

Figure 4.3: Relative protein expression levels of upstream IRF3 targets in Ictidomys tridecemlineatus liver tissue throughout the torpor-arousal cycle. (A) Histogram showing mean standardized expression levels ( \pm S.E.M., $n=4$ independent protein isolations from different animals). (B) Representative western immunoblot bands illustrating the expression levels of all targets. Data were analyzed using a one-way ANOVA followed by a Tukey post-hoc test $(p<0.05)$. For each parameter measured, values sharing the same letter notation are not statistically different from one another. 
(A)

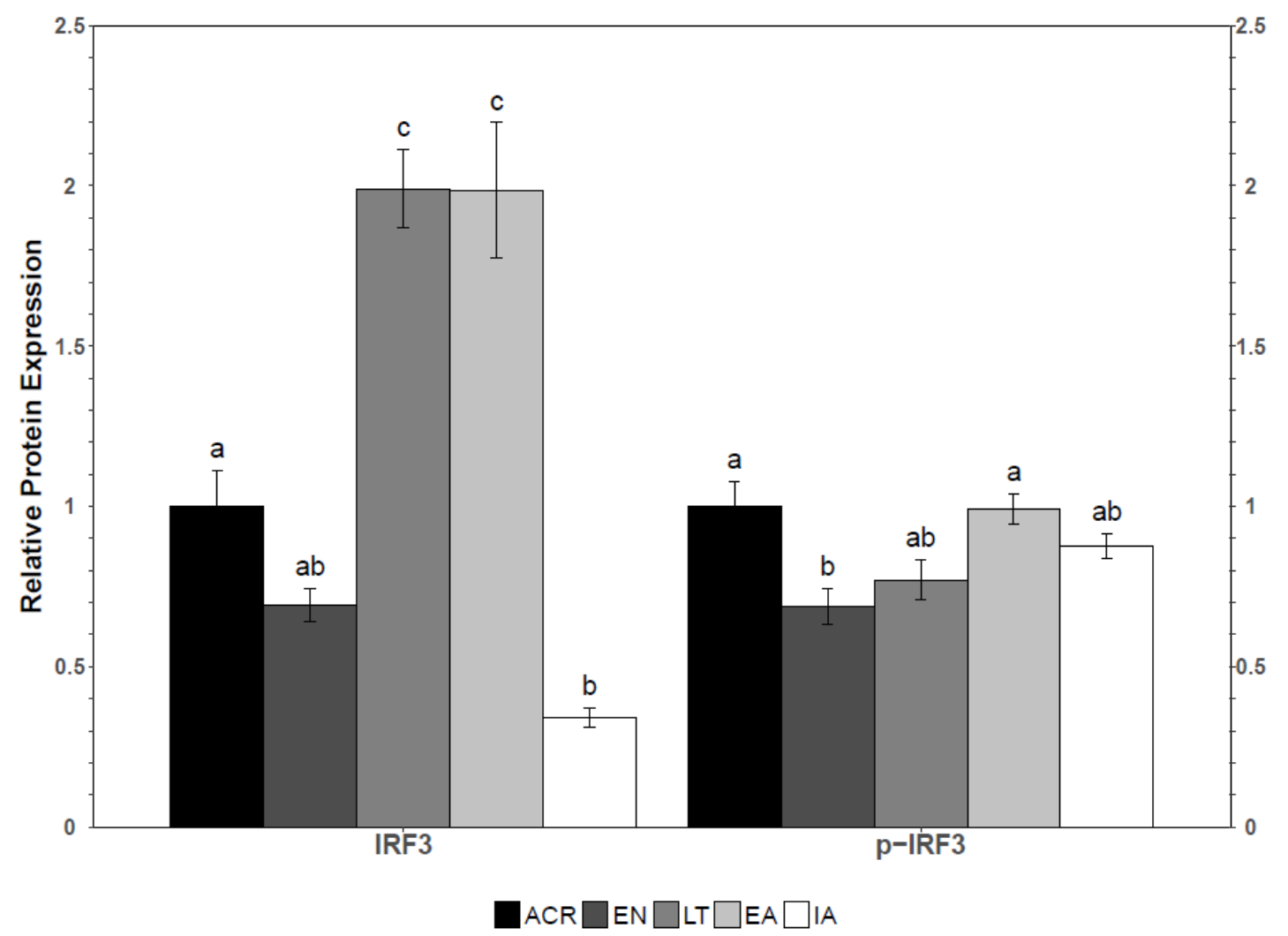

(B)

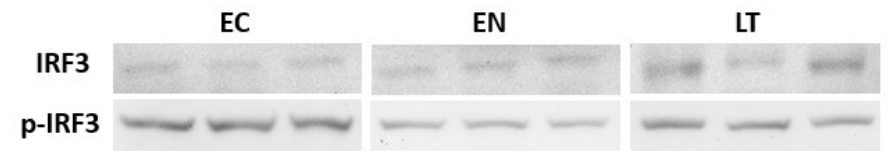

EA

IA

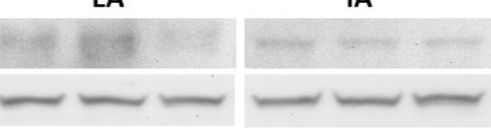

Figure 4.4: Relative protein expression levels of total IRF3 and phosphorylated IRF (Ser396) in Ictidomys tridecemlineatus brown adipose tissue throughout the torpor-arousal cycle. (A) Histogram showing mean standardized expression levels of IRF3 and p-IRF3 ( \pm S.E.M., $\mathrm{n}=4$ independent protein isolations from different animals). (B) Representative western immunoblot bands illustrating the expression levels of IRF3 and p-IRF3. Data were analyzed using a one-way ANOVA followed by a Tukey post-hoc test $(p<0.05)$. For each parameter measured, values sharing the same letter notation are not statistically different from one another. 
(A)

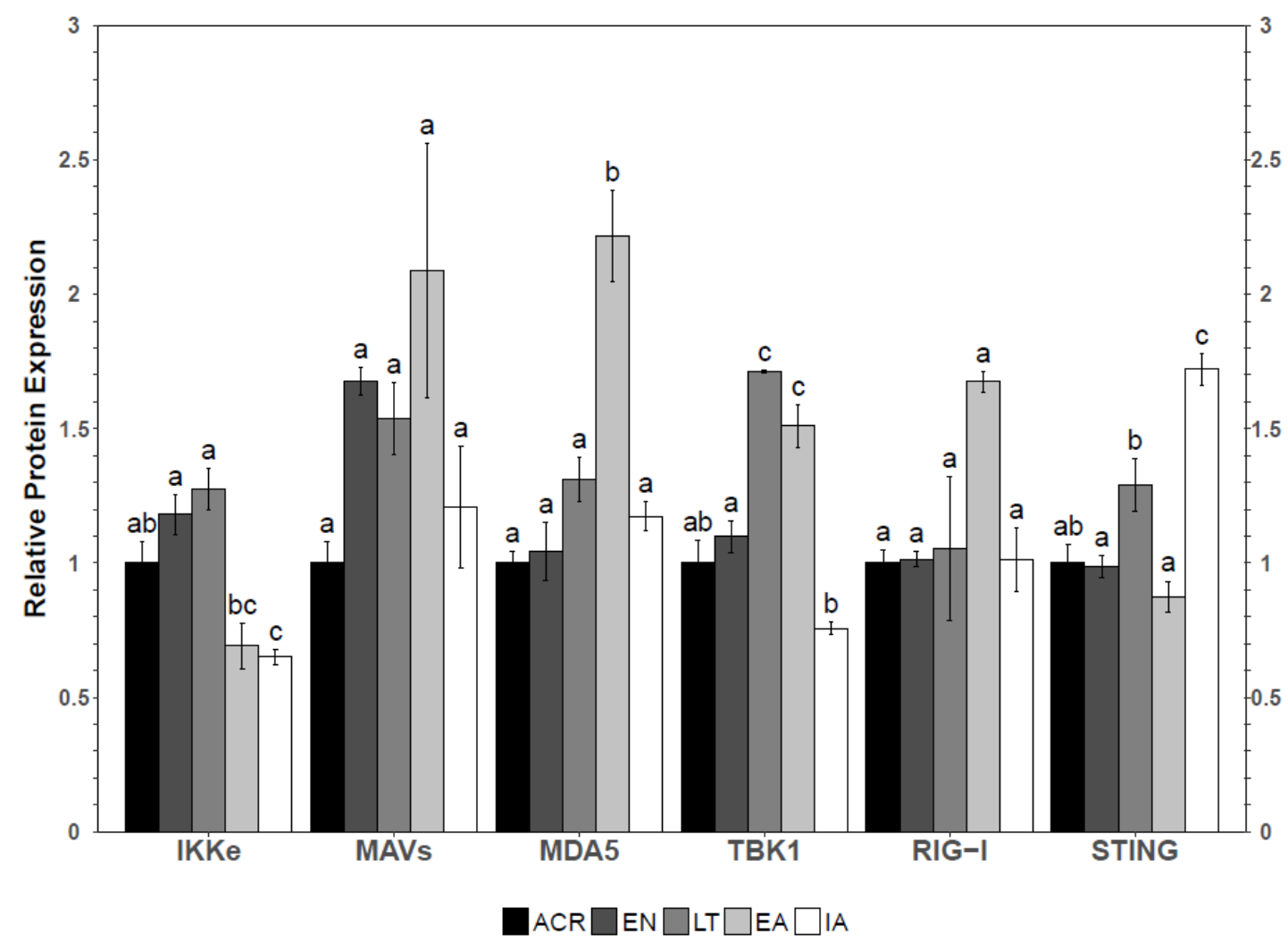

(B)

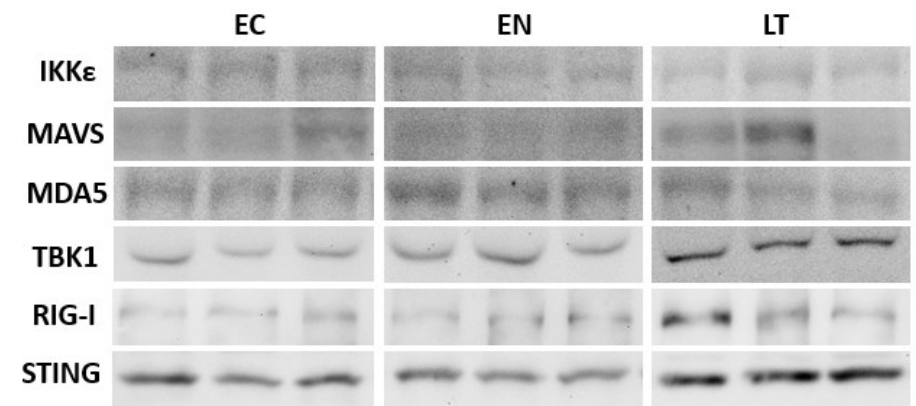

EA

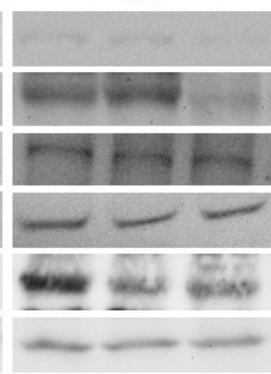

IA

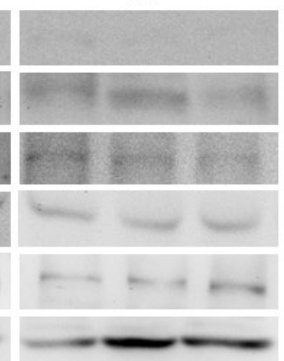

Figure 4.5: Relative protein expression levels of upstream IRF3 targets in Ictidomys tridecemlineatus brown adipose tissue throughout the torpor-arousal cycle. (A) Histogram showing mean standardized expression levels ( \pm S.E.M., $n=4$ independent protein isolations from different animals). (B) Representative western immunoblot bands illustrating the expression levels of all targets. Data were analyzed using a one-way ANOVA followed by a Tukey post-hoc test $(p<0.05)$. For each parameter measured, values sharing the same letter notation are not statistically different from one another. 
(A)

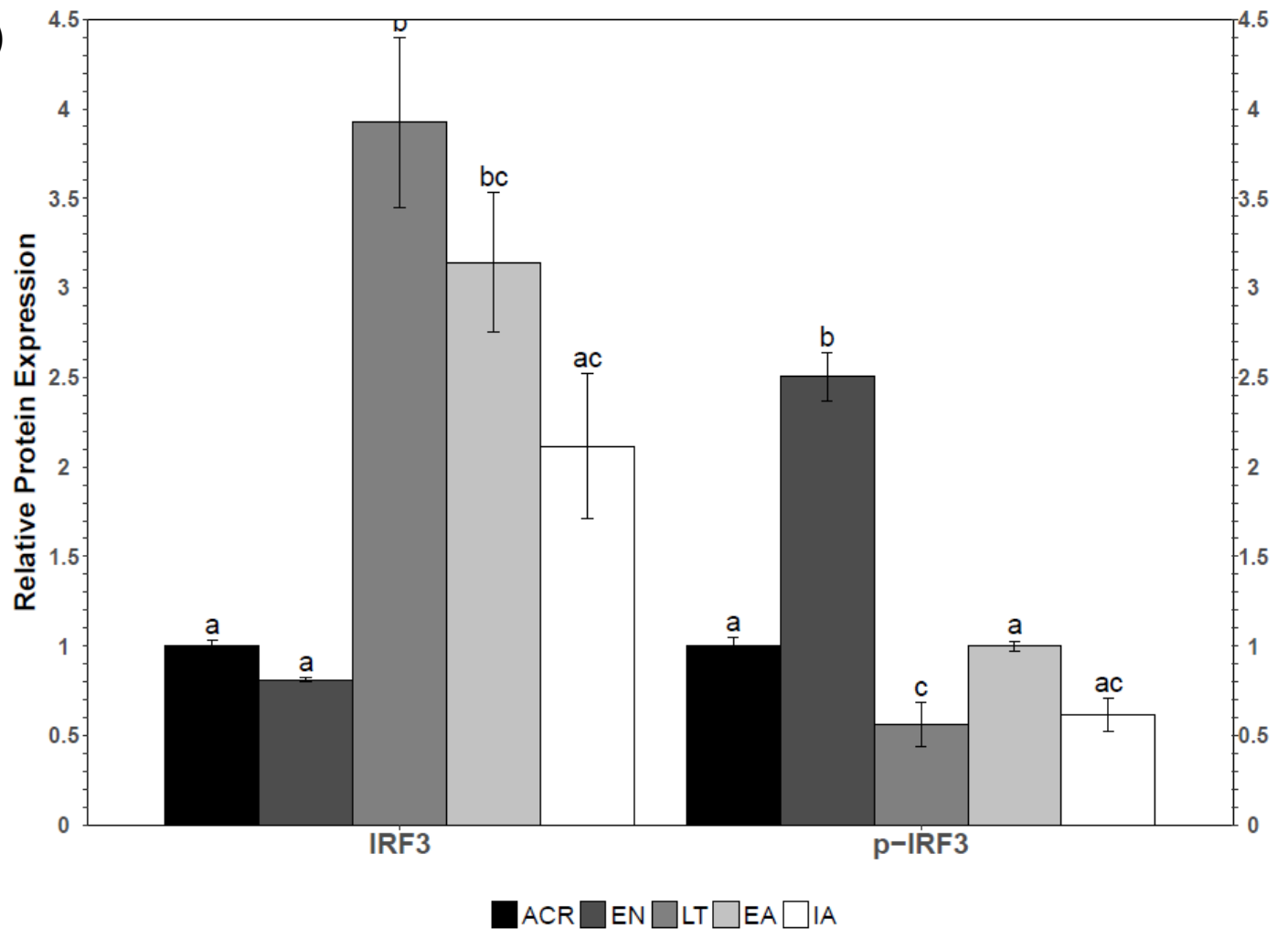

(B)

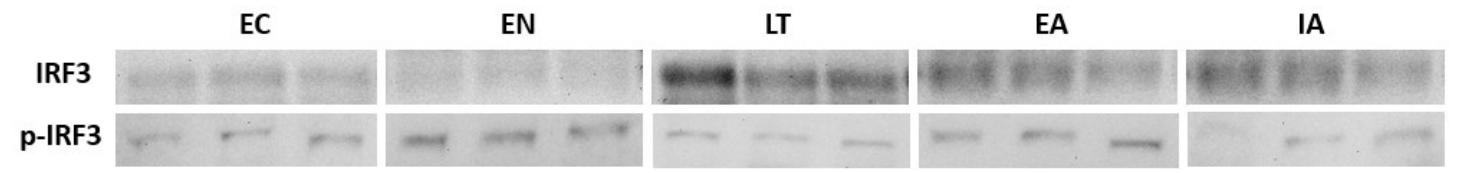

Figure 4.6: Relative protein expression levels of total IRF3 and phosphorylated IRF (Ser396) in Ictidomys tridecemlineatus white adipose tissue throughout the torpor-arousal cycle. (A) Histogram showing mean standardized expression levels of IRF3 and p-IRF3 ( \pm S.E.M., $\mathrm{n}=4$ independent protein isolations from different animals). (B) Representative western immunoblot bands illustrating the expression levels of IRF3 and p-IRF3. Data were analyzed using a one-way ANOVA followed by a Tukey post-hoc test $(p<0.05)$. For each parameter measured, values sharing the same letter notation are not statistically different from one another. 
(A)

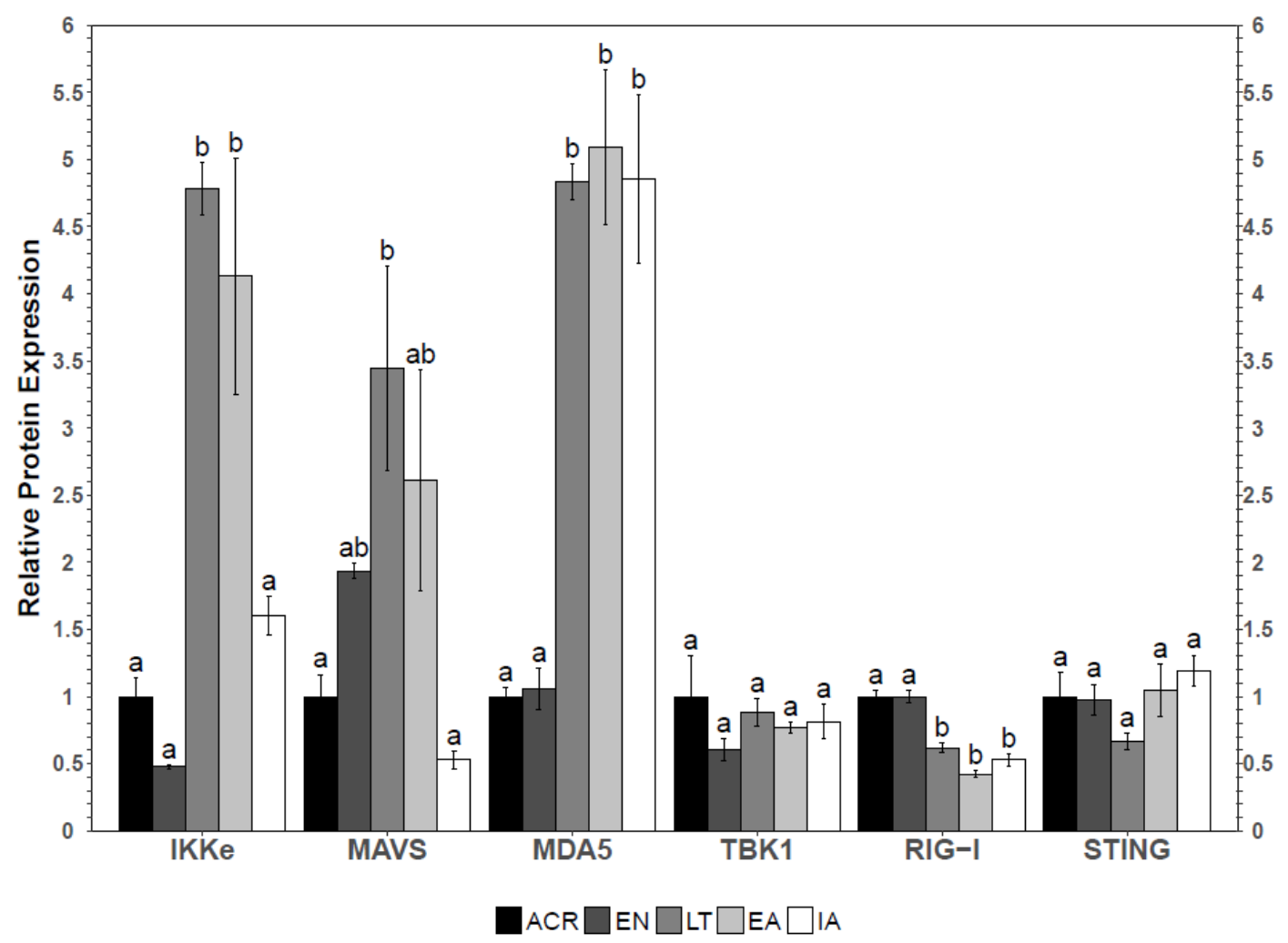

(B)

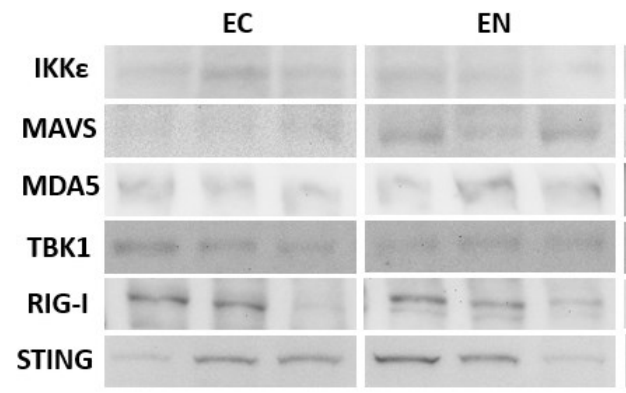

LT

EA

IA
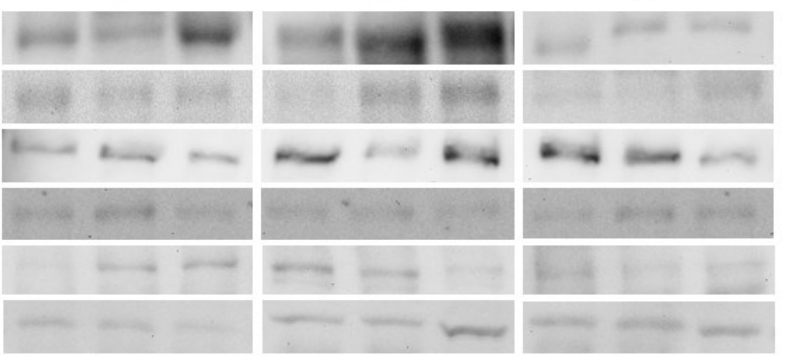

Figure 4.7: Relative protein expression levels of upstream IRF3 targets in Ictidomys tridecemlineatus white adipose tissue throughout the torpor-arousal cycle. (A) Histogram showing mean standardized expression levels ( \pm S.E.M., $\mathrm{n}=4$ independent protein isolations from different animals). (B) Representative western immunoblot bands illustrating the expression levels of all targets. Data were analyzed using a one-way ANOVA followed by a Tukey post-hoc test $(p<0.05)$. For each parameter measured, values sharing the same letter notation are not statistically different from one another. 


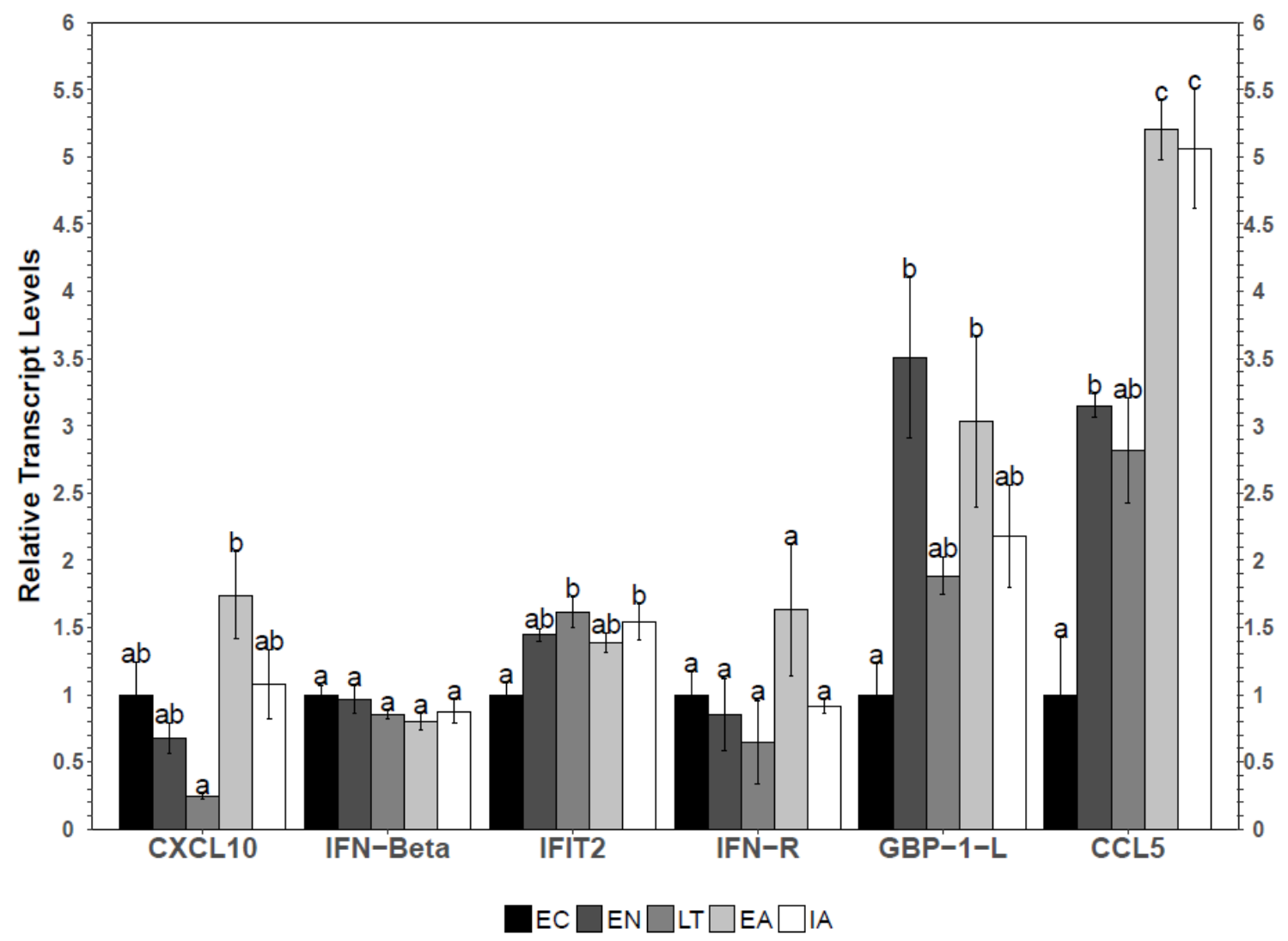

Figure 4.8: Histogram of normalized IRF3 downstream gene transcript levels in Ictidomys tridecemlineatus liver tissue throughout the torpor-arousal cycle. Data are mean critical threshold $(\mathrm{Ct})$ ( \pm S.E.M. $n=4$ independent trials on tissue from different animals). Values that share the same letter are not significantly different $(p<0.05)$. 


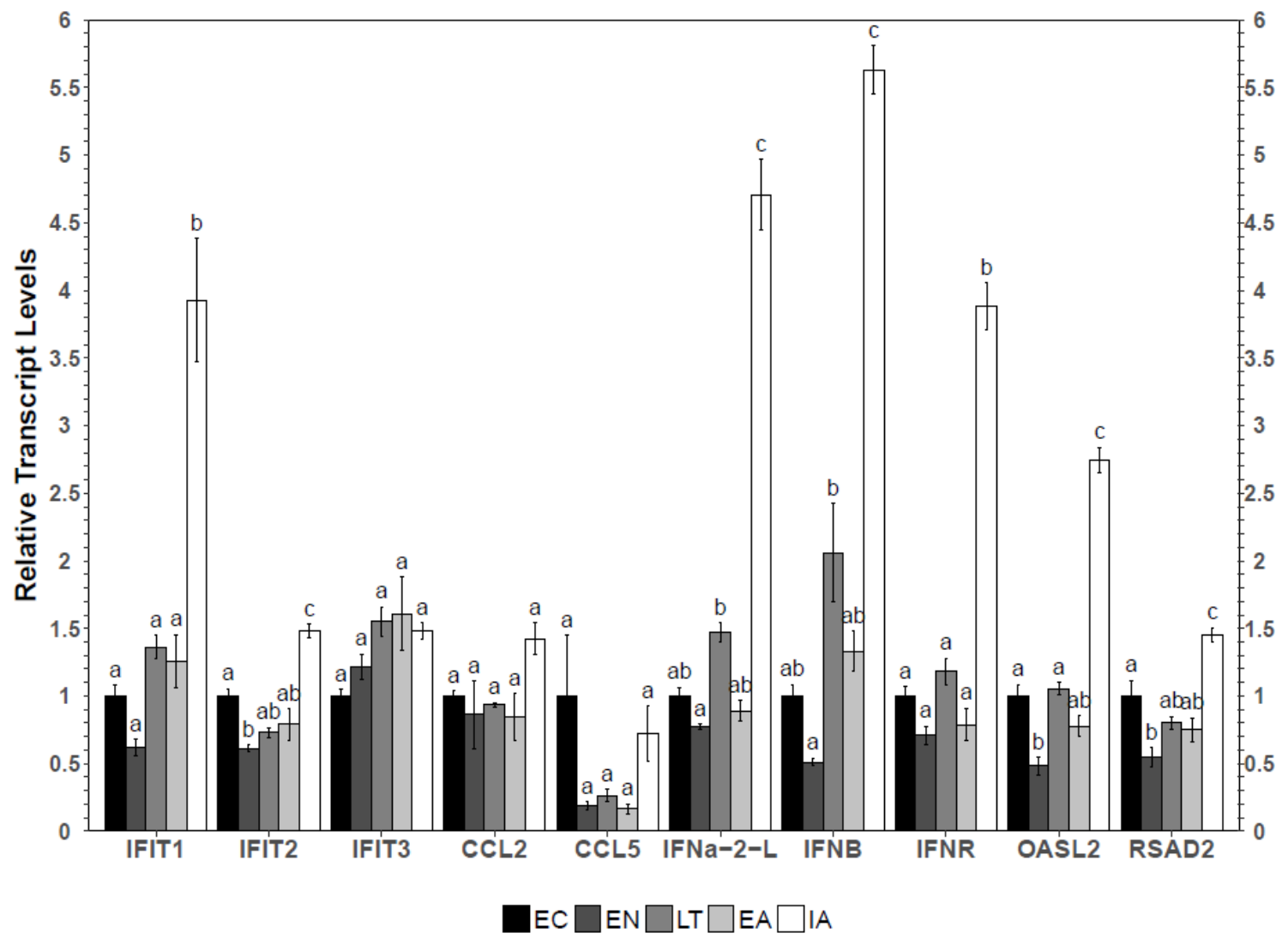

Figure 4.9: Histogram of normalized IRF3 downstream gene transcript levels in Ictidomys tridecemlineatus brown adipose tissue throughout the torpor-arousal cycle. Data are mean critical threshold $(\mathrm{Ct})$ ( \pm S.E.M. $n=4$ independent trials on tissue from different animals). Values that share the same letter are not significantly different $(p<0.05)$. 


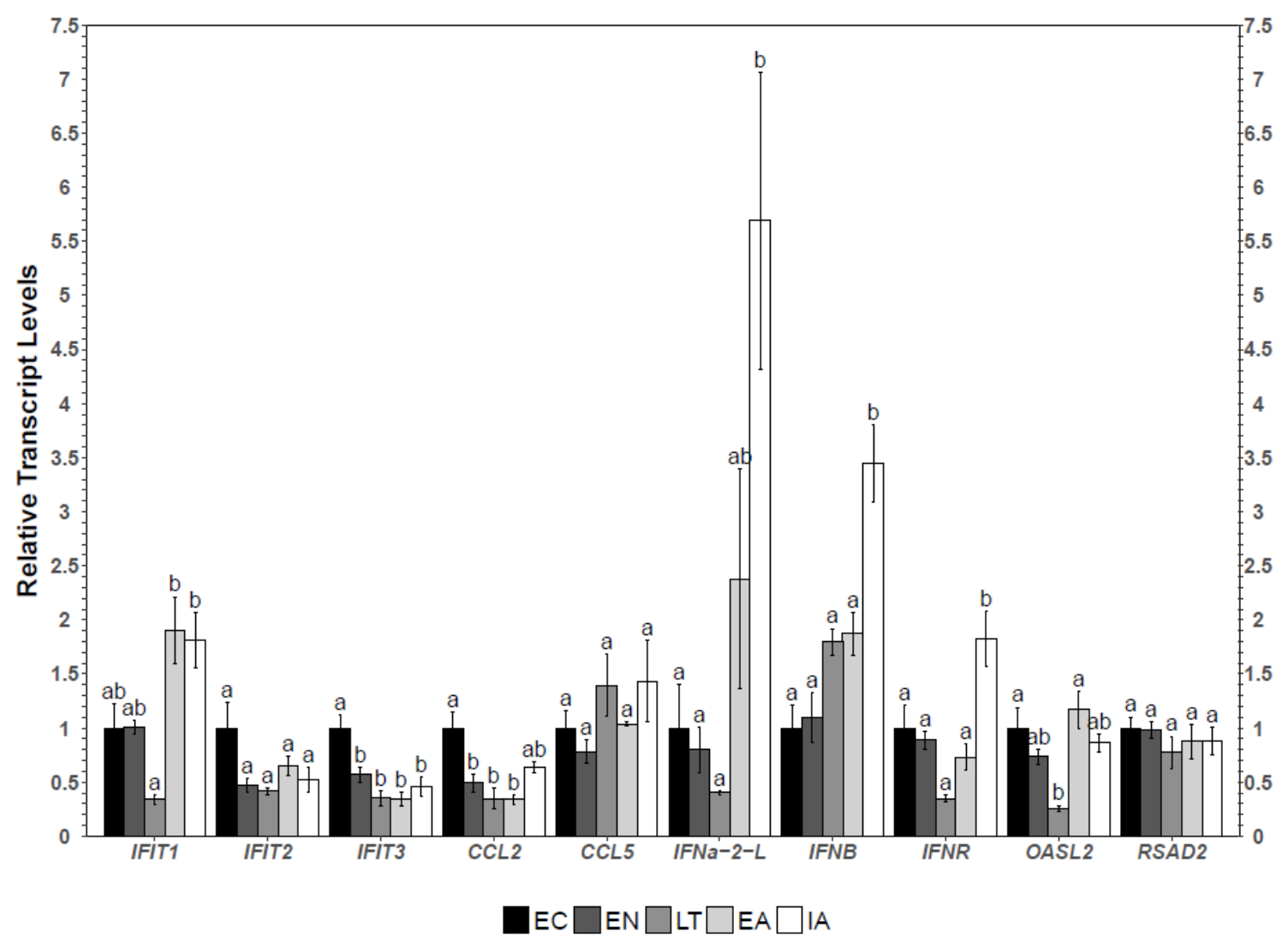

Figure 4.10: Histogram of normalized IRF3 downstream gene transcript levels in Ictidomys tridecemlineatus white adipose tissue throughout the torpor-arousal cycle. Data are mean critical threshold $(\mathrm{Ct})( \pm$ S.E.M. $n=4$ independent trials on tissue from different animals). Values that share the same letter are not significantly different $(p<0.05)$. 
Appendix 4.1 Primer design for RT-qPCR

\begin{tabular}{ccc}
\hline Gene name & Forward Primer 5' $-\mathbf{3}^{\prime}$ & Reverse Primer 5' $\mathbf{- 3}$ \\
\hline$I R F 3$ & TCCGATCCTCAGCTAAACGC & ATCGGAAGGAAGTGTTGCGT \\
\hline$I F I T 1$ & TTCTATGCGGGGTTTGCCAT & CTGGACAGCCTTTCTCAGGG \\
\hline$I F I T 2$ & TCACCTGGGGAAACTATGCC & AAGTTGGCTTCTGGGCTCTT \\
\hline$I F I T 3$ & GGCAACCACTTCAACCACAC & GATTCCCTCCCGCTTGTGA \\
\hline$C C L 2$ & GGTTGAGGACTACGTTGCCA & TACAGGTTCTGGGGTGGTGA \\
\hline$C C L 5$ & GCTCCAACCTAGCAGTTGTCT & CATTTCTTCTCTGGGTCGGC \\
\hline$C X C L 10$ & AACTCAAGGAATGCCGCTCT & CCCCGCTCTTTTCATTGTGG \\
\hline$I F N a-2-L$ & GGACTTTGCCTTCCCCAAGGAGCAG & GCTGGGTCCCACAGGCTTTCAGGTC \\
\hline$I F N-$ Beta & CAAAAGCTGGGGAAACTCCA & TTCCATTCGGATCACTGTCCA \\
\hline$I F N-$ Receptor & AAGGGGTCAAGAACTGGGTC & CCTCCAATTTGCAGCGTACC \\
\hline GBP- $-L$ & TGTGCCTCATCCCAAGATGG & TCATTCTGGTTGTCGCCCTT \\
\hline$O A S L 2$ & TGCATTGGAGCTACTGACCAT & CACAAGCCCTTCGTCCATGT \\
\hline$R S A D 2$ & AAACTTCTCTGGCGGAGAGC & ACCGTAGTCCCTGAACCACT \\
\hline
\end{tabular}


Appendix 4.2. IRF3 pathway from Peng et al., 2015. PLOS pathogens. 10(4):e1004041

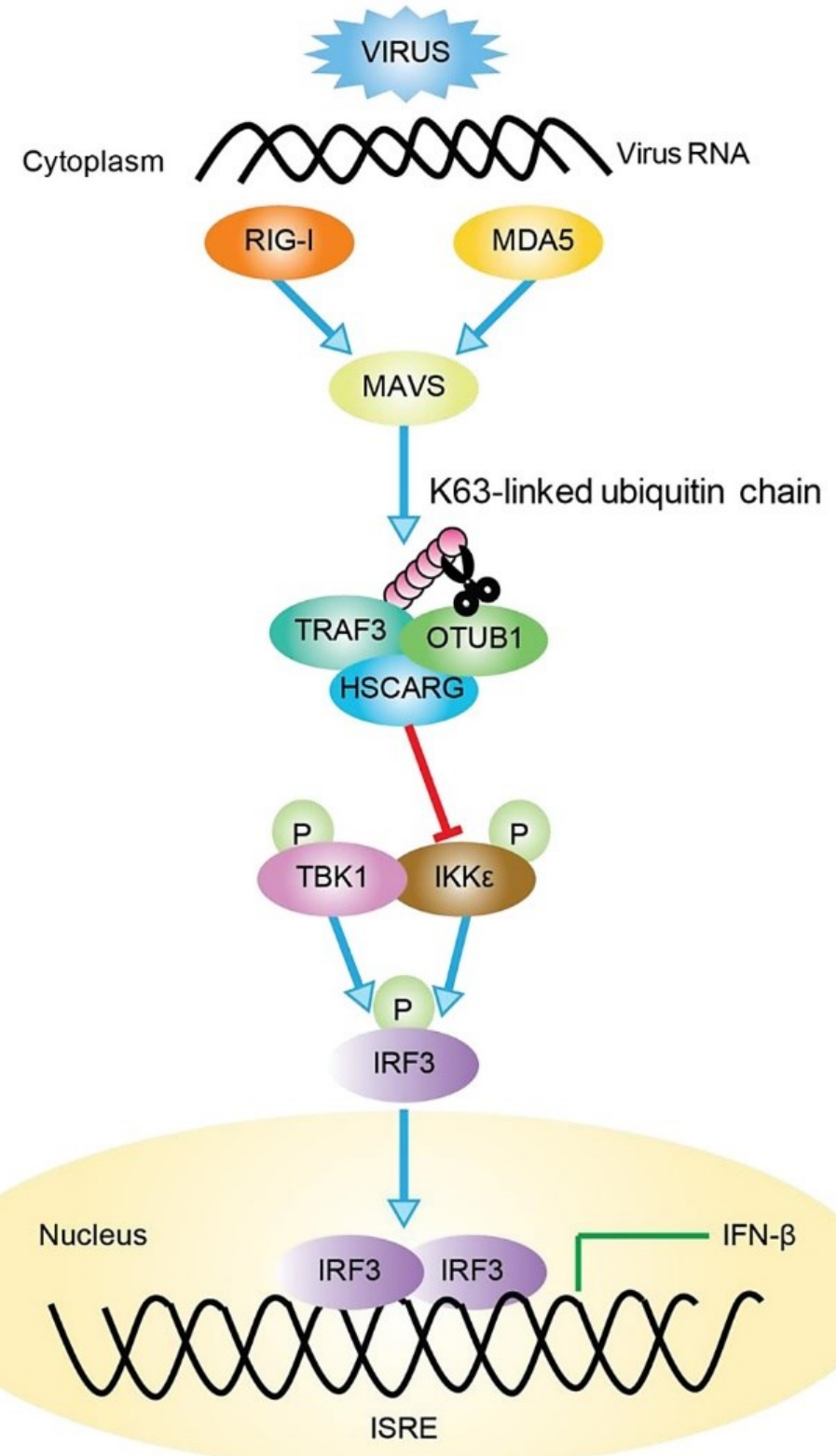




\section{CHAPTER 5}

\section{IDENTIFICATION OF A PRO-SURVIVAL NEUROPROTECTIVE PEPTIDE IN A MAMMALIAN HIBERNATOR; S-HUMANIN}

Manuscript in press; accepted 2019-06-05

Szereszewski KE, Storey KB. 2019. Identification of a prosurvival neuroprotective mitochondrial peptide in a mammalian hibernator. Cell Biochemistry \& Function. 2019; 1-10. https://doi.org/10.1002/cbf.3422 


\begin{abstract}
Hibernation requires the intricate regulation of physiological and biochemical adaptations to facilitate the decrease in metabolic rate and activation of pro-survival factors needed for winter survival. Mitochondria play important roles in eliciting these responses and in coordinating the required energy shifts. Herein, we report the presence of a novel mitochondrial peptide, s-humanin, in the hibernating thirteen-lined ground squirrel, Ictidomys tridecemlineatus. S-humanin was shown to have strong structural and sequence similarities to its human analogue, humanin - a powerful neuroprotective mitochondrial peptide. An assessment of the protein and gene expression levels of this peptide in ground squirrels revealed stark tissue-specific regulatory responses whereby transcript levels increased in brain cortex, skeletal muscle, and adipose tissues during hibernation, suggesting a protective torpor-induced activation. Accompanying peptide measurements found that s-humanin levels were suppressed in liver of torpid squirrels, but enhanced in brain cortex. The enhanced transcript and protein levels of s-humanin in brain cortex suggest that it is actively involved in protecting delicate brain tissues and neuronal connections from hibernation-associated stresses. We propose that this squirrel-specific peptide is involved in modulating tissue-specific cytoprotective functions, expanding its role from human-specific neuroprotection to environmental stress-protection.
\end{abstract}




\subsection{Introduction}

To survive cold winter months many small mammals, such as ground squirrels, use hibernation. Responding to the high metabolic cost of maintaining a euthermic body temperature of $\sim 37^{\circ} \mathrm{C}$ in the cold, they retreat into a torpid state and allow their core body temperature $\left(\mathrm{T}_{\mathrm{b}}\right)$ to drop to near ambient (often to near $0^{\circ} \mathrm{C}$ ), and their metabolic rate to fall to just $1-5 \%$ of euthermic values $[14,17,18]$. Hibernators cycle through prolonged periods of torpor (sometimes lasting several weeks) that are interspersed with brief periods of arousal when both metabolic rate and $\mathrm{T}_{\mathrm{b}}$ return to euthermic values. Arousal is costly since it requires high rates of thermogenesis (mainly by brown adipose tissue), blood reperfusion and oxygen consumption as well as imposing oxidative stress on organs $[18,205,206]$ but overall, by hibernating, animals can accrue metabolic energy savings of $\sim 90 \%$ as compared with the cost of maintaining euthermia over the winter $[14,18]$. However, to achieve the hypometabolic state of torpor requires significant reorganization of an animal's metabolism, including a switch to a primary reliance on body lipid reserves for fuel, a suppression of ATP-expensive metabolic processes (ion pumps, transcription, translation, etc.) and an enhancement of cell preservation strategies (antioxidant defenses, chaperones, other pro-survival mechanisms, etc.).

Hibernation is not only fascinating by itself but mammalian hibernators are also useful model systems with which to explore molecular mechanisms that could ameliorate various human neurological disorders including ischemic injury and Alzheimer's disease (AD) [207-210]. In AD, for example, plaques formed by Amyloid-beta (A $\beta$ ) and neurofibrillary tangles contribute to the degradation of neurons in the brain. Another feature of AD is the accumulation of oxidative damage to DNA, RNA, protein and lipids [211, 212]; indeed, $\mathrm{A} \beta$ itself shows oxidative damage in amyloid plaques. However, in hibernating ground squirrels, cerebral blood flow can decrease to as low as $10 \%$ of euthermic values, yet animals avoid any significant brain injury [207]. The lack of damage is due in part to the very low metabolic rate and deep hypothermia of the hibernating state that can attenuate neurodegeneration - but hibernation also includes inducible protective measures against cellular damage such as enhanced chaperone levels, antioxidants and anti-apoptotic mechanisms $[17,18,119,207]$. Another interesting feature of hibernation is that neural 
structures (e.g. spines, synaptic structures, dendritic branches) all decrease in complexity, size and number during torpor [213, 214] but are regenerated (synaptogenesis) during arousal with no cognitive loss [215-218]. In this way, ground squirrels are able to successfully enter and exit from hibernation without neuronal injury or death.

While searching for pro-survival factors that could aid resistance to apoptosis in the unaffected brain areas of a patient with Alzheimer's disease (AD), research by the Nishimoto lab in Japan discovered a novel peptide that they proposed was implicated in neuroprotection. This peptide, named humanin, is encoded within the $16 \mathrm{~S}$ ribosomal RNA gene of the mitochondrial genome (MT-RNR2) and is 24 or 21 amino acids in length depending on the location of the translation machinery (cytosolic vs. mitochondrial) [61, 219, 220]. The secreted peptide (amino acid sequence: 5'MAPRGFSCLLLLTSEIDLPVK-3') has been shown to have anti-apoptotic effects [221] and antagonize neurotoxicity and various types of Alzheimer's disease genes [222]. When translated in the mitochondrion, the resulting humanin peptide lacks the three carboxyterminal amino acids but remains fully functional. The amino acid structure includes a positively charged $\mathrm{N}$-terminal domain, a central hydrophobic region, and a polar $\mathrm{C}$ terminal domain, which conforms to the general design of signaling peptides [223]. The 3D model of humanin shows a flexible structure with definite turn points that allow it to interact with potential receptors whereas its central alpha-helical section (Gly5 to Leu18) provides for specific interactions and/or passage through hydrophobic environments such as membranes [223]. Residues 3-19 have also been described as the "core domain" of the peptide and are responsible for its neuroprotective activity [224]. Modification of core domain amino acids was found to yield analogues resulting in increased potency, affect binding to growth-factor binding proteins, as well as affect its own secretion [225].

It has been proposed that humanin can suppress $\mathrm{AD}$ by reducing neuronal cell death caused by $A \beta$ accumulation [226] and oxidative damage [227-230]. Humanin exerts this effect by mediating the remodeling of dendritic structural complexities and by preventing mitochondrial dysfunctions. This effect is mediated via the activation of the JAK2/STAT3 pathway, triggering the inhibition of AD-related neurotoxicity [231], and in part by ERK and PI3K pathway activation, as seen in mice [232]. In addition to its neuroprotective 
effects (also including improving memory and learning) [233-235], humanin has been proposed to have several cytoprotective effects, including protection against oxidative stress, atherosclerotic plaque formation, stimulating pancreatic beta-cell and insulin sensitivity, and suppressing apoptosis. Apoptotic effects have been well characterized [65, $221,225,228,233,235-242]$; they include protection from $A \beta$-induced toxicity of cerebrovascular smooth muscle cells and by interacting with insulin-like growth factorbinding protein 3 (IGFBP3) where humanin blocks IGFBP3-induced apoptosis in glial cell lines [231].

Given that humanin is an important neuroprotective agent, we hypothesized that a ground squirrel homologue could play a significant role in neuroprotection of the brain during hibernation and that the peptide might aid cytoprotection in other tissues as well. The present study demonstrates the presence of a ground squirrel homologue "s-humanin" peptide in tissues of hibernating thirteen-lined ground squirrels (Ictidomys tridecemlineatus), and evaluates the tissue-specific regulation of gene and protein expression of the squirrel peptide over the torpor-arousal cycle. The data revealed tissuespecific regulation at both the protein and transcript levels suggesting that s-humanin is in fact involved in various neuroprotective and cytoprotective mechanisms facilitating squirrel hibernation.

\subsection{Materials and Methods}

\subsubsection{Model prediction}

The 3D model prediction for the squirrel peptide was accomplished using the I-Tasser server [243-245] (http://zhanglab.ccmb.med.umich.edu/I-TASSER/), a hierarchical template-based method of protein structure and function prediction. Structure prediction is performed based on three principles: template identification, full-length structure assembly, and structure-based function annotation. To determine s-humanin structure, the predicted s-humanin nucleotide sequence was translated by the vertebrate mitochondrial code (giving 5'-MAPRGFSCLLLLTSEIDLPVK -3') and was then inputted into the I- 
Tasser server. Briefly, target sequences were searched through the Protein Data Bank (PDB) structure library to find possible folds. Continuous fragments were excised from the aligned regions and were used to reassemble full-length models while unaligned regions were built by ab initio modeling. The predicted model has a helical structure involving residues Phe6 to Leu9 and an overall turn-like structure between residues Gly5 and Ile16. Various scoring values were implemented to determine the best possible model. These PDB models were validated with a secondary structure to enhance reliability of the predicted model. Finally, a Levitt-Gerstein LG score and a MaxSub score were compared for likelihood of model probability. The humanin 3D model (PDB ID: 1Y32) was then aligned to the predicted squirrel-specific sequence using the molecular visualization software PyMOL.

\subsubsection{Animal protocols}

Thirteen-lined ground squirrels (Ictidomys tridecemlineatus) were captured, treated, and organs harvested following the same protocol as previously described in Chapter 2 [102]

Animal experiments were conducted as previously described [102]. All animal experiments were conducted by in the laboratory of Dr. J.M. Hallenbeck lab (NINDS, Bethesda, Maryland) and approved by the Institutional Animal Care and Use Committee. Equal numbers of male and female squirrels ( $\sim 5-10)$ approximately between 1 and 3 years of age were sampled for this experiment and an $n=4$ squirrels were used for each experimental condition. Samples of liver, muscle, brown adipose tissue (BAT), white adipose tissue (WAT), brain cortex (BCTX) and brainstem (BSTEM) were collected and were flash-frozen in liquid nitrogen, shipped on dry ice to Carleton University, and then stored in $-80^{\circ} \mathrm{C}$ until use.

\subsubsection{Quantitative PCR}

RT-qPCR was used to examine the relative expression of s-humanin gene transcripts. RNA extraction was performed as previously described [188]. An aliquot of $171 \mu \mathrm{L}$ 
autoclaved water was added to final each RNA sample, and then serial dilutions of $10^{-2}$ and $10^{-3}$ were made. Forward and reverse primers for $s$-humanin were designed based on the consensus sequence of human humanin sequence (Genbank accession no. AY029066.1) and the ground squirrel mitochondrial genome (Genbank accession no. KP698974) using the NCBI primer design program. Primers were synthesized by Sigma Genosys. Primer sequences for s-humanin were: forward 5' - TGCCCAGTGACACACGTTTA - 3' and reverse 5' - AGCTCCATAGGGTCTTCTCGT - 3'. Tissue-specific housekeeping genes were used as controls; tbp for brown adipose tissue (BAT); $\alpha$-tubulin for liver (LIV); $\gamma$ actin for skeletal muscle (MUS); $\alpha$-actin for white adipose tissue (WAT); and gapdh for brain stem (BSTEM) and brain cortex (BCTX). Amplification was performed as described previously [188] using a Bio-Rad CFX Connect thermocycler. A 2-fold dilution series standard curve of pooled samples was run for each quantification run. Thermocycling consisted of an initial denaturing stem at $95^{\circ} \mathrm{C}$ for 5 minutes, followed by 40 cycles of denaturing at $95^{\circ} \mathrm{C}$ for $10 \mathrm{~s}$, annealing at $60^{\circ} \mathrm{C}$ for $20 \mathrm{~s}$, and extension at $72^{\circ} \mathrm{C}$ for $20 \mathrm{~s}$. Postrun melt analyses were also run from 55 to $95^{\circ} \mathrm{C}$ for all reactions to determine reaction efficiencies. Critical threshold $(\mathrm{Ct})$ values for each animal condition (EC, EN, ET, LT, EA, and IA) were standardized to the $\mathrm{Ct}$ of the respective housekeeping gene from the same sample. The statistical comparative threshold cycle $\left(\Delta \Delta \mathrm{C}_{\mathrm{t}}\right)$ method was used to determine relative gene expression for each tissue whereby mRNA quantification was done by calculating the ratio of starting quantity of target gene to the reference gene of each sample. Data are expressed as relative mean expression $( \pm \mathrm{SEM})$ for $n=4$ independent samples from different animals. Statistical analysis of the data was performed using a one-way ANOVA followed by a post-hoc Tukey test to identify significant differences among groups. Statistical difference was accepted if $p<0.05$. For each parameter measured, values that were not statistically different from one another share the same letter notation. All statistical analysis and Figure generation was performed using RBioplot [104].

\subsubsection{Protein extraction and ELISA assay}

Total protein extracts of ground squirrel tissues were prepared according to manufacturer's instructions for the human putative humanin peptide (MT-RNR2) ELISA kit from CUSABIO (Cat \# CSB-EL015084HU). Briefly, frozen tissue samples of $\sim 50 \mathrm{mg}$ 
each were quickly rinsed with $1 \mathrm{X}$ PBS buffer and then homogenized in $1 \mathrm{~mL}$ of $1 \mathrm{X}$ PBS and stored overnight at $-20^{\circ} \mathrm{C}$. After two short freeze-thaw cycles, homogenates were centrifuged for $5 \mathrm{~min}$ at $5,000 \mathrm{~g}$ at $4^{\circ} \mathrm{C}$. The supernatant was removed and assayed immediately.

All reagents and standards were prepared according to manufacturer's instructions from stock reagents supplied with the kit including preparation of 7 serial dilutions of reconstituted standard $(28-1800 \mathrm{pg} / \mathrm{mL})$ plus a blank (sample diluent). For assay, $100 \mu \mathrm{l}$ aliquots of extracts of EC and LT samples, standards and blank were loaded into wells of the manufacturer-supplied microplate, then covered with an adhesive strip and incubated for $2 \mathrm{~h}$ at $37^{\circ} \mathrm{C}$. Next, the liquid was removed from each well and $100 \mu \mathrm{L}$ of biotin antibody (1X) was added. Wells were then covered with a new adhesive strip and incubated for 1 hour at $37^{\circ} \mathrm{C}$. Each well was then aspirated and washed $3 \mathrm{X}$ with $200 \mu \mathrm{L}$ of wash buffer for 2 minutes. Next, $100 \mu \mathrm{L}$ of HRP-avidin (1X) was added to each well and incubated for $1 \mathrm{~h}$ at $37^{\circ} \mathrm{C}$. Wells were washed as previously described for $3 \times 2$ min and then $90 \mu \mathrm{L}$ of TMB substrate was added to each well and incubated for $15-30 \mathrm{~min}$ at $37^{\circ} \mathrm{C}$, protected from light. Next, $50 \mu \mathrm{L}$ of stop solution was added to each well and then the optical density was measured spectrophotometrically at $450 \mathrm{~nm}$ with background correction set to $540 \mathrm{~nm}$. Mean standardized values for peptide abundance $\pm \operatorname{SEM}(n=4$ independent tissue extracts

from different animals) were calculated. Data were analyzed using the Student's $t$-test to identify significant differences between EC and LT conditions. All statistical analysis and figure generation was performed using RBioplot [104].

\subsection{Results}

\subsubsection{Sequence complementarity}

The predicted I. tridecemlineatus s-humanin mitochondrial gene sequence was determined via sequence complementarity with the humanin mitochondrial sequence by performing a Blastn search of the 13-lined ground squirrel mitochondrial genome (Genbank accession no. KP698974.1). The predicted nucleotide sequence obtained was $88 \%$ complementary to that of humanin (Fig. 5.1A) and was also encoded within the $16 \mathrm{~S}$ rRNA portion of the squirrel mitochondrial genome [246]. Next, the predicted s-humanin 
amino acid sequence (translated via the vertebrate mitochondrial code) was determined and aligned with the sequences from other mammals obtained from the NCBI data bank: golden-mantled ground squirrel (Callosperophilus lateralis, Accession No. KP698975.1), Norway rat (Rattus norvegicus, Accession No. KF011917.1), little brown bat (Myotis lucifugus, KT901455.1), David's myotis bat (Myotis davidii, Accession No. KM233172.1), domestic dog (Canis familiaris, Accession No. AY729880.1), cow (Bos taurus, Accession No. NC_006853.1), Swinhoe's striped squirrel (Tamiops swinhoei, Accession No. KP027416.1), Siberian chipmunk (Tamias sibiricus, Accession No. KF668525.1), naked mole rat (Heterocephalus glaber, Accession No. HQ689652.1) and the red squirrel (Sciurus vulgaris - Accession No. AJ238588). All sequences and compared to humanin using the multiple sequence alignment tool in Clustal Omega (Fig. 5.1B).

Since the peptide can move into the cytoplasm or be retained in the mitochondria for translation, its sequence will differ depending on the location of the translation machinery. Despite its small size, and likelihood for sequence conservation across species, humanin is in fact not present in all mammalian species. Some animals have been shown to express more than one form of the peptide whereas others express it only from one subcellular compartment. This study aimed to illustrate the sequence conservation of humanin among various mammals as well as its translational origin (Fig. 5.1B). Furthermore, particular peptide regions are highly conserved across species, whereas in other regions some bases vary, even among species from similar taxa. Hence, the versatility of the peptide can be seen, even across species of relatively recent evolutionary divergence (Fig. 5.1B). The variation across mammalian species is also illustrated by sequences with a letter " $\mathrm{M}$ " indicating the shorter mitochondrial peptides translated with the vertebrate mitochondrial code, whereas sequences with a letter " $\mathrm{R}$ " indicate the longer isoforms translated in the cytoplasm with the standard genetic code. The predicted s-humanin peptide showed a high degree of conservation to Homo sapiens humanin and the peptide in other mammalian species; indeed, amino acid positions 1, 2, 4, 5, 6, 8-11 and 14-21 were identical in all species (Fig. 5.1B). The most highly variable site was positon 3 where 8 different amino acids occurred among the 10 species listed. Positions 7, 12, and 13 showed variability in some species as compared with the ground squirrel sequence but conformed at other sites. 
Except for position 3, the sequence from the golden-mantled ground squirrel C. lateralis (another hibernator) was identical with that of I. tridecemlineatus.

\subsubsection{Homology Modeling}

A predicted 3D model of the s-humanin peptide was generated using the bioinformatics homology modeling program I-Tasser. The NMR structure of humanin (Fig. 5.2A) was obtained from the RCSB protein data bank online system (1 y32 NMR structure of Humanin in 30\% TFE solution). The predicted model of s-humanin was obtained from the community-wide Critical Assessment of Structure Prediction (CASP) tool and the I-Tasser program using the predicted amino acid sequence (Fig. 5.2B). A scoring function (C-score) based on relative clustering structural density and consensus score of multiple threading templates was used to estimate the accuracy of the I-Tasser predictions. Details for the ITasser model prediction are explained in [243]. The current model was analyzed based on an LG score and a MaxSub score. LG scores $>4.0$ are evaluated as extremely good models, and $>1.5$ as fairly good. MaxSub scores $>0.8$ are evaluated as extremely good models, and $>0.1$ as fairly good. S-humanin was found to have an LG score of 4.396 and a MaxSub score of 0.680 indicating a high reliability as a very good model.

\subsubsection{S-humanin transcript levels}

Relative transcript levels of s-humanin were assessed using quantitative PCR (qPCR) to examine gene expression in multiple tissues of 13-lined ground squirrels across six points of the torpor-arousal cycle. Sampling points were: euthermic in the cold room (EC), entrance into torpor (EN), early torpor (ET), late torpor (LT), early arousal (EA) and interbout arousal (IA) (see Figure 1.1 for more detail). Figure 5.3 shows that s-humanin transcript levels did not change in liver across the torpor-arousal cycle whereas the other tissues showed significant changes. In skeletal muscle, a $1.49 \pm 0.13$-fold increase was noted in s-humanin gene transcripts as squirrels entered torpor (compared with EC) but transcript levels were reduced again across the rest of the cycle. In brown adipose tissue (BAT), a significant $3.00 \pm 0.40$-fold increase in s-humanin transcripts occurred during entrance into torpor, followed by a return to near-EC (control) values over the remainder 
of the cycle. White adipose tissue (WAT) showed a different pattern with transcript levels rising strongly during torpor to peak during $\mathrm{LT}$ at $2.88 \pm 0.25$-fold over EC values. This was followed by a strong drop during early arousal to just $0.32 \pm 0.08 \%$ (as compared with EC) before returning to control levels during interbout arousal. S-humanin transcript levels were also assessed in two regions of the brain (cortex and brainstem) comparing EC and LT conditions (Fig. 5.4). No significant change was noted in the brainstem, but a significant increase in transcript levels to $1.66 \pm 0.12$-fold in LT was noted in the cortex compared with EC.

\subsubsection{S-humanin protein levels}

Relative protein levels of s-humanin were also assessed in the six tissues of $I$. tridecemlineatus using an enzyme-linked immunosorbent assay (ELISA) to compare euthermic controls (EC) and animals in prolonged torpor (LT). Figure 5.5 shows that shumanin levels changed significantly in two tissues: liver and brain cortex. Liver showed a significant decrease in s-humanin content during torpor with values falling to $0.80 \pm$ $0.01 \%$ as compared with EC. A significant increase in expression was noted in the brain cortex by $1.48 \pm 0.13$-fold but no significant change occurred in the brainstem. An upward trend was noted in both adipose tissues, but changes were not significantly different from euthermic values. Finally, no significant change was noted in skeletal muscle between EC and LT conditions.

\subsection{Discussion}

\subsubsection{S-humanin and sequence homology}

The present study investigated the tissue-specific regulation and structural model prediction of a previously unknown peptide from thirteen-lined ground squirrels, shumanin. The human homologue of this peptide, humanin, was first reported in 2001 [222] and in subsequent years has been shown to have multiple neuroprotective and cytoprotective actions $[61,220]$. S-humanin may have analogous actions that could serve specific protective roles during the winter hibernation of ground squirrels. The examination 
of mRNA transcript levels, protein levels, sequence homology and 3D model prediction of s-humanin presented here was determined based on sequence similarity to humanin. This is supported by evidence from the current study that found an $88 \%$ sequence homology of the s-humanin gene to humanin, and a similar RMSD value of $3.317 \AA$.

Comparison of the squirrel peptide homologue to other species revealed interesting findings, showing that most amino acid residues were fully conserved between these mammalian species whereas others that were quite variable (Fig. 1). The characteristics of the variant amino acids revealed a functional pattern that was also noted in a previous studies [225, 247]. The region between proline 3 to proline 19 was described as the "core domain" of humanin that is essential for the secretion of the full-length peptide from the cell despite the wide variability at position 3 between species. The function of individual residues has also been well characterized: the phenylalanine at position 6 as well as lysine at position 21 were found to be essential for binding to IGFBP-3 [225, 239, 247]; residues $3,7-9,12-14$, and 19 were crucial for pro-apoptotic function [59, 222, 225]; and amino acids at positions 9-11, 19 and 20 were found to be critical for humanin secretion [225, 247] revealing that the specific sequence of this short peptide is key to its various cytoprotective functions. Further studies have shown a structural/functional role for the cysteine residue at position 8 (conserved in all species). When this cysteine was replaced with an alanine, peptide activity was lost. However, when the amino acid at position 14 (serine) was replaced by a glycine, a 1000-fold increase in pro-apoptotic activity was noted $[60,240]$. Therefore, the specific amino-acid sequence of the peptide appears to play an important role in its regulation. Our findings suggest another layer of regulation that may depend on the amino acid position 3; this residue appears to provide species-specificity, being different for each of the animals analyzed (Fig. 5.1B) and showing that its conservation among evolutionary groups does not appear to be very stringent [248]. We originally postulated that sequence conservation would be higher among similar groups such as the ground squirrel hibernators compared to non-hibernators or among recently diverged mammalian groups. Indeed, the sequence of the hibernating golden-mantled ground squirrel (C. lateralis) differed from that of I. tridecemlineatus only at position 3 and the hibernating chipmunk (T. sibiricus) differed only at position 12 but note that all three of these species also belong to the subfamily Xerinae of the family (Sciuridae). 
However, the hibernating bats (M. lucifugus and M. davidii) also showed just 2 differences from the s-humanin sequence such that high sequence conservation may exist among these species that all drop their $\mathrm{T}_{\mathrm{b}}$ to low temperatures during hibernation, but this was not always the case [246, 249-251].) Non-hibernators including human, cow, dog, red squirrel and naked mole rat all showed three or more sequence differences from s-humanin (except rat and striped squirrel had just two).

\subsubsection{Tissue Regulation}

At the protein level, humanin has been shown to be a key player in various survival pathways such as anti-apoptosis, antioxidants and neuroprotection during periods of stress $[59,227,230,231]$. As a homologue of humanin, we propose that s-humanin likely also participates in the activation of protective pathways during hibernation. In humans, humanin activation is dependent on its secretion from key tissues (e.g. testes, colon, brain and skeletal muscle) [61] and transport via the circulation to other tissues which is accomplished by peptide binding to the carrier protein insulin-like growth factor-binding protein 3 (IGFBP-3) [59, 239]. S-humanin transcripts and protein levels were detected in multiple tissues: liver, skeletal muscle, white and brown adipose and brain. Both gene and protein expression showed differential tissue-specific regulation over the torpor-arousal cycle. Liver s-humanin may not have a crucial role across the hibernation cycle since transcript levels were generally unchanged and protein levels actually decreased. However, muscle expression of s-humanin RNA was induced during entrance into torpor followed by a decrease in early torpor and subsequent return to control values. Additionally, protein levels were unchanged in response to torpor suggesting that perhaps the entrance period is most crucial for the initial induction of this cytoprotective peptide in muscle in preparation for prolonged torpor.

Brown and white adipose tissues (BAT and WAT) showed significant elevation of shumanin transcript levels during entrance into torpor in BAT (increased $\sim 3$-fold) as well as during early torpor through to late torpor in WAT (rising $\sim 3$-fold) compared to EC 
values. The strong upregulation of transcripts during entrance into torpor suggests that the s-humanin peptide might have its greatest impact during the entrance and early torpor phases. However, peptide levels appeared elevated (but not significantly) in late torpor in both adipose tissues. These increases in s-humanin gene expression in adipose tissues could be indicative of an induction of protective pro-survival pathways. In fact, both of these tissues are crucial to hibernation success. BAT is the primary thermogenic organ that rewarms the squirrel body during arousal from torpor using non-shivering thermogenesis fueled by high rates of fatty acid oxidation by uncoupled mitochondria in BAT [205, 252, 253]. The very high oxygen consumption of BAT during arousal as well as the increased oxygen consumption of all organs as they return back to euthermic function also leads to high levels of reactive oxygen species (ROSs) generated during the arousal period [7]. This is validated by the precipitous drop in the levels of plasma ascorbate (a powerful antioxidant) over the same timeframe [206]. Humanin has been shown to be a key modulator of oxidative stress via the induction of antioxidant enzymes such as SOD [227, 231, 254] and glutathione peroxidase [226] as well as mediating chaperone-mediated autophagy [230], suggesting a comparable action for s-humanin. Additionally, studies have shown that oxidative stress is widely involved in the development of various neuropathologies that can be counteracted by the effects of humanin. Therefore, it is probable that s-humanin plays a significant role in BAT over the hibernation cycle in the mitigation of oxidative stress.

White adipose tissue is also crucial to hibernation success because it is the main storage depot of lipids that are the primary fuel supporting survival over the non-feeding winter months [253]. However, WAT is also a significant player in the immune system and secretes various pro- and anti-inflammatory agents during periods of stress [255]. The increase in s-humanin expression noted in WAT was nearly 3-fold in late torpor followed by a significant drop to $\sim 0.32$ during arousal. This could be the result of a role in inflammatory regulation. Humanin has been shown to regulate inflammatory processes through the activation of the formyl-peptide receptor-like 1 and 2 (FPRL1 and FPRL2) which mediate the immune response as well as chemotaxis [227, 256]. Humanin acts as a ligand for the FPRL receptors which relay signals through the ERK 1/2 signaling cascade to suppress the induction of inflammatory agents and the proliferation of adipocytes [42, 
227]. In the hibernating squirrel, p-ERK $1 / 2$ protein levels were previously shown to decrease significantly during entrance, late torpor and arousal in WAT to $\sim 0.3,0.1$ and 0.2 fold of control values, respectively [42], suggesting a suppression of the immune response and/or adipocyte growth during prioritized energy savings.

Lastly, two sections of ground squirrel brain were assessed comparing control (EC) and late torpor (LT) conditions: brainstem and brain cortex. The cortex showed a significant upregulation of both s-humanin transcript ( $\sim 1.66$-fold) and protein $(\sim 1.5$-fold $)$ levels in torpid animals. This suggests that torpor stimulated an increase in the amount of s-humanin produced (and potentially secreted) from the brain cortex. Humanin was originally discovered as a neuroprotective agent that suppressed $A \beta$-related pathologies as well as tau plaque formation in Alzheimer disease of the brain [60, 228, 231] but many other protective functions have now been described. The brain cortex is known to be important role in cognitive control such as higher order thinking, goal-oriented planning, motivation, sensory input control, etc. [257]. By contrast, the brainstem is responsible for more autonomic functions. Our study showed elevated s-humanin transcript and protein levels in the cortex, in contrast to the brainstem that showed no change, and this suggests that enhanced protection of the cortex area of the brain is key to neuroprotection during torpor [258]. We suggest that because ground squirrels must maintain superior neuronal plasticity and synaptogenesis over multiple torpor bouts [215-218], that this region has likely prioritized for neuroprotection, compared to the brainstem region whose functions are known to be suppressed during torpor (e.g. controls over respiration, heart rate, $\mathrm{T}_{\mathrm{b}}$ and circadian rhythm).

Additionally, it has been shown that hibernating ground squirrels do not appear to show any significant neurodegeneration upon exiting from a torpor bout, making the squirrel an excellent model to contribute to the study of neurological disorders such as Alzheimer's [15]. As previously mentioned, the neurons in squirrel brain retract and reduce in size and complexity during torpor but regain their original morphology when torpor ends, permitting the squirrel to retain cognitive and motor functions upon arousal. The neuroprotective actions of humanin may be crucial to this through actions that could control and coordinate various pathways. These could include (a) attenuation of neuronal cell death 
via inhibition of the pro-apoptotic Bcl-2 family member, Bax (hindering its translocation from the cytosol into the mitochondria) [221, 239], (b) modulation of oxidative damage via induction of antioxidant enzymes such as SOD through the ERK signaling cascade or via stimulation of the PI3K and the JAK/STAT pathways [60, 226, 239], or (c) suppression of amyloid- $\beta$-induced tau hyperphosphorylation (one of the characteristic features of $\mathrm{AD}$ pathology) by inhibiting the phosphorylation of Tyr307 on protein phosphatase 2A (PP2A), thereby causing its activation [226]. Therefore, increases in both s-humanin transcript and protein levels in the cortex are highly indicative of its protective function for areas of highlevel cognition and survival.

In summary, this study describes s-humanin as the ground squirrel-specific homologue of humanin, proposing cyto- and neuro-protective functions in adipose tissues and brain cerebral cortex. We propose that these mechanisms are upregulated at different times across the torpor-arousal cycle of hibernation in these tissues to assist long-term survival and to suppress oxidative or inflammatory damage. Therefore, s-humanin appears to be multi-faceted protective mitochondrial peptide that may be very important for the coordination of survival pathways during hibernation in the thirteen-lined ground squirrel. 


\section{Figures}

A

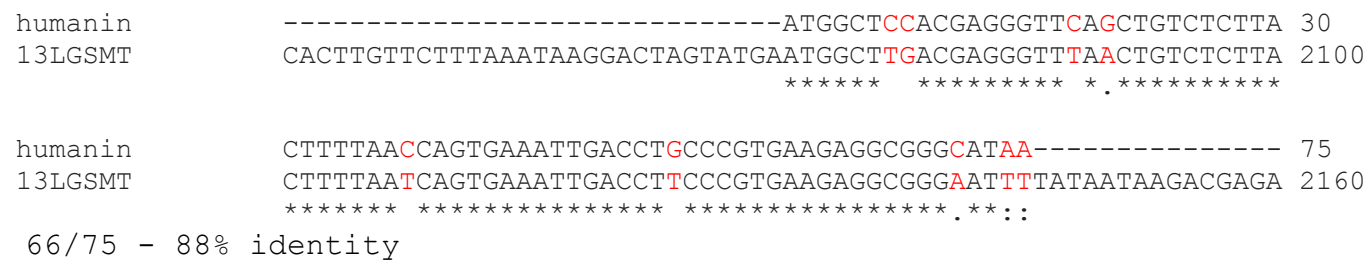

B

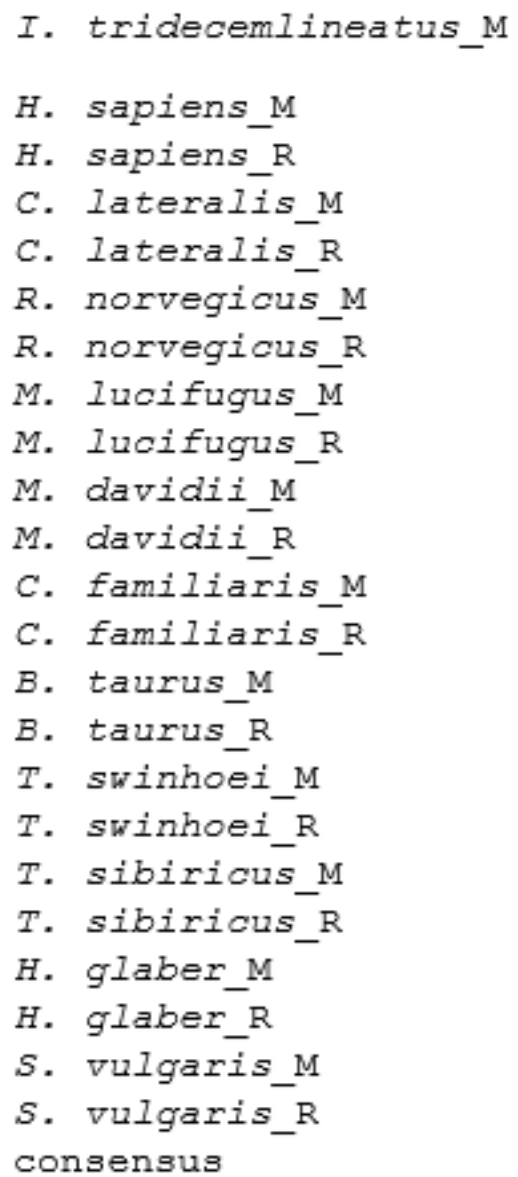

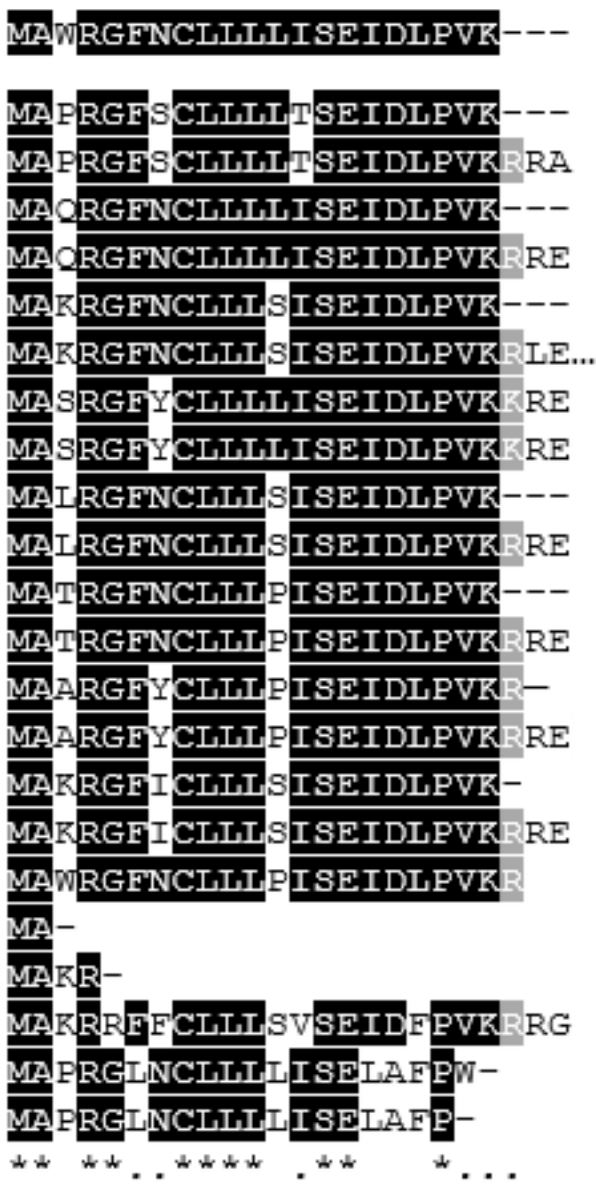

Fig. 5.1. Multiple sequence alignment of the predicted homologues of humanin peptide encoded by MT-RNR2 and MT-RNR2-like mitochondrial and nuclear genes in several mammalian species. (A) Alignment of human humanin and predicted S-humanin gene sequences. (B). Alignment the amino acid sequences of mammalian species done in Clustal Omega and rendered graphically by BOXSHADE 3.21. Identical amino acid residues are highlighted in black and similar amino acids are highlighted in gray. " $\mathrm{M}$ " indicates the shorter predicted mitochondrial isoforms translated with the mitochondrial genetic code, whereas " $R$ " indicates the longer predicted nuclear isoforms translated with the standard genetic code. Species names and accession numbers for sequences are defined in the Methods (section 5.2). 
A

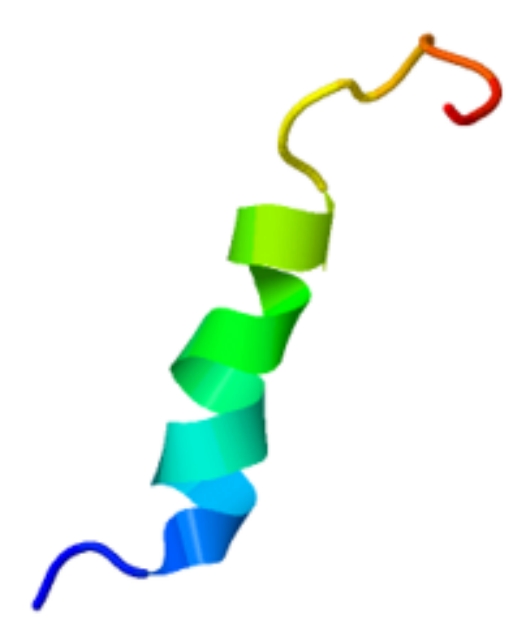

B

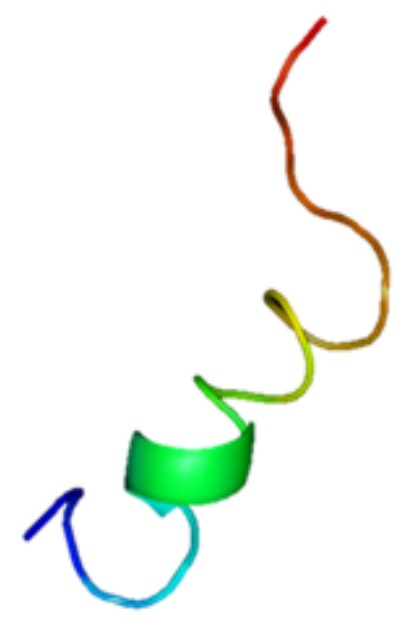

Fig. 5.2. Homology modeling of humanin (A) and predicted S-humanin (B) performed using the I-Tasser program with the human PDB code as the primary template. 


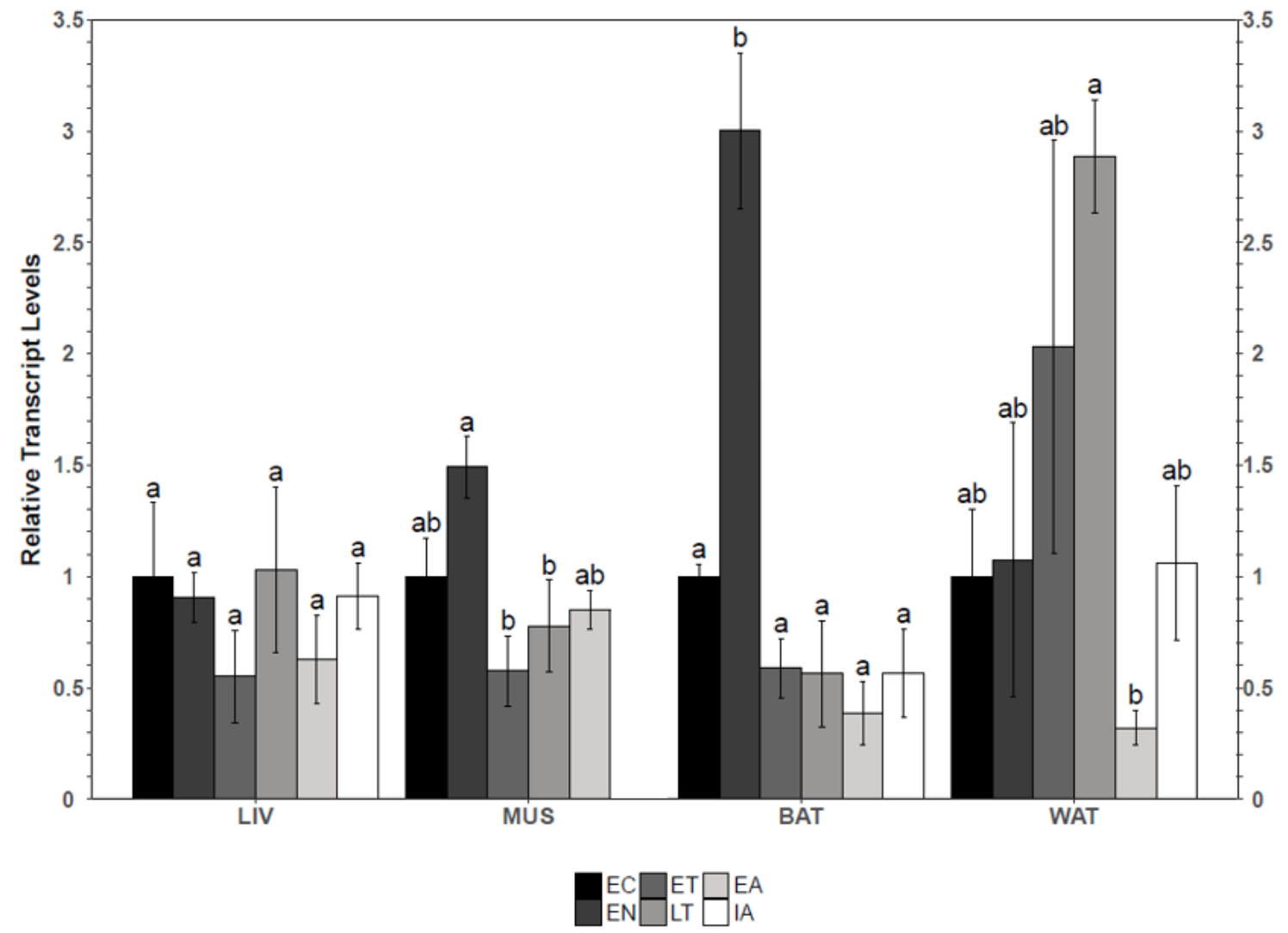

Fig. 5.3. Histogram of standardized s-humanin gene transcript levels across the torporarousal cycle in liver (LIV), skeletal muscle (MUS), brown adipose tissue (BAT) and white adipose tissue (WAT). Data are mean critical threshold $(\mathrm{Ct}) \pm \operatorname{standard}$ error $(n=4$ independent trials on tissue from different animals). Values that share the same letter are not significantly different $(p<0.05)$. 


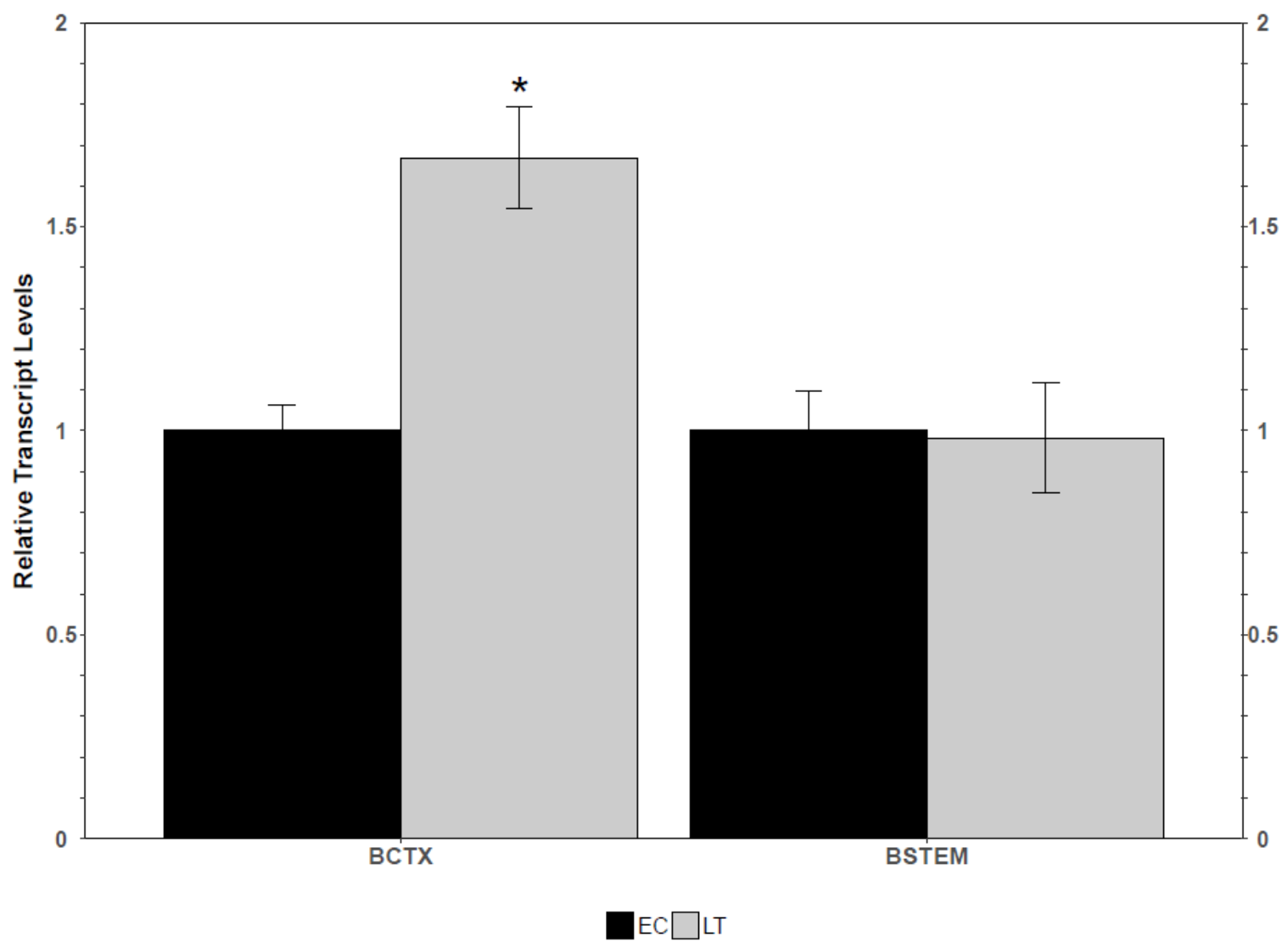

Fig. 5.4. Histogram of standardized s-humanin relative transcript levels under EC and LT conditions in brain cortex (BCTX) and brainstem (BSTEM). Data are mean critical threshold $(\mathrm{Ct}) \pm$ standard error $(n=4$ independent trials on tissue from different animals). Asterisk $(*)$ denotes a significant change compared to control levels $(p<0.05)$. 


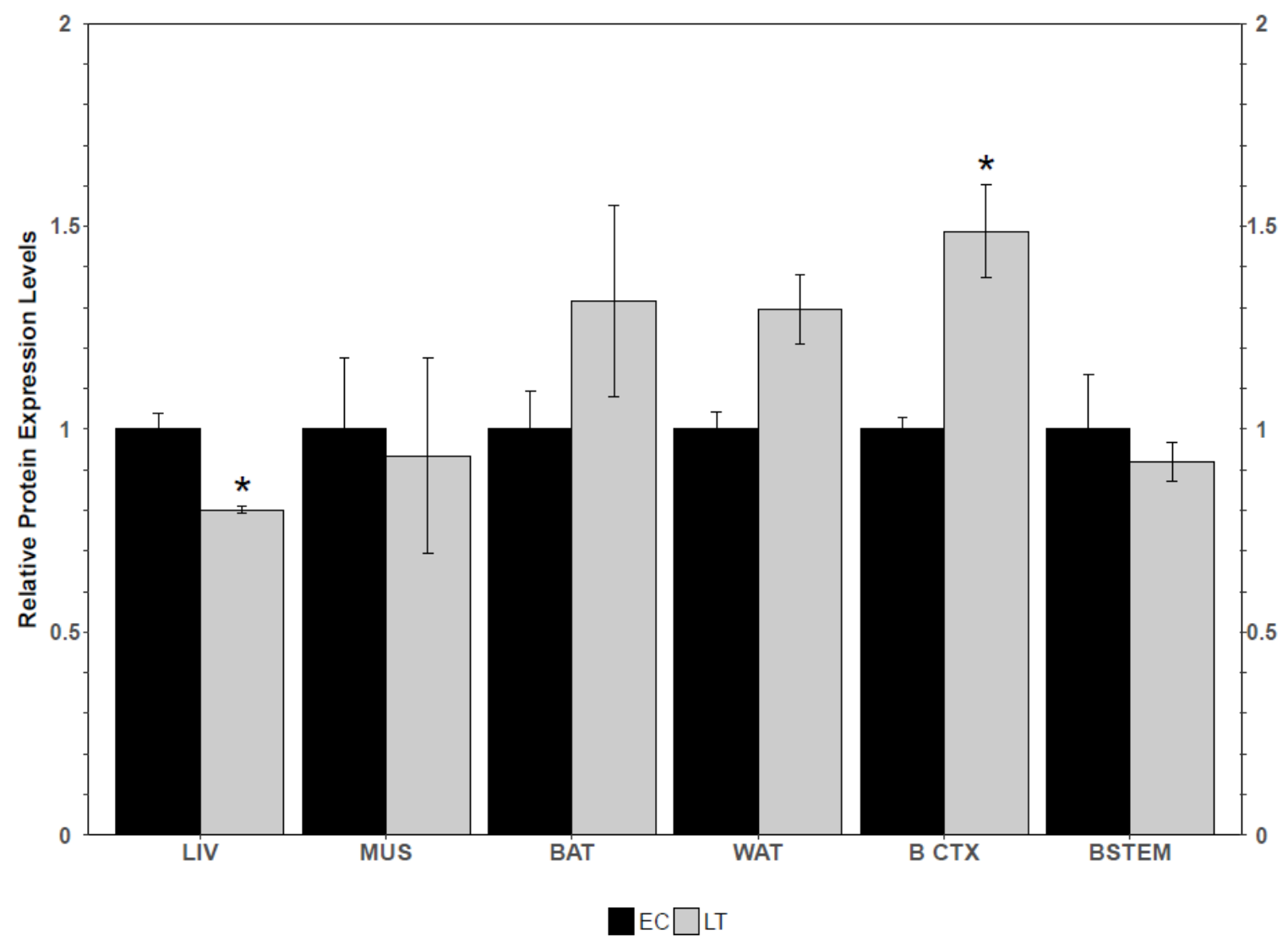

Fig. 5.5. Histogram of standardized s-humanin relative protein levels in control and late torpor. Data are mean \pm standard errors $(n=4$ independent trials on tissue from different animals). Asterisk $(*)$ denotes a significant change compared to control levels $(p<0.05)$. 


\section{CHAPTER 6}

\section{GENERAL DISCUSSION}




\subsection{General Discussion}

The concept of hibernation has been extensively studied using various species of mammals as models. Knowledge gained from studies of the underlying metabolic regulation of hibernation has permitted several advancements in fields of medical research including applications to organ transplantation, repair of organ dysfunction, treatment of disease, and the potential for suspended animation for space travel [259] and has been remarkably useful in the treatment of various diseases such as in the case of cardiac arrest. In 2015, surgeons began clinical trials on the use of profound hypothermia (mimicking hibernation) to lower a patient's body temperature in order to maintain viability for over two hours without any circulating blood after a significant hemorrhage [260]. Less extreme hypothermia has been employed in another surgical procedure where a surgery performed on the aortic arch required blood to be rerouted through a heart-lung machine [261]. It was shown that lowering the patient's core body temperature enabled surgeons to maintain brain viability without circulation [262]. Nowadays, the routine use of mild hypothermia $\left(33-34^{\circ} \mathrm{C}\right)$ is used on patients who suffered cardiac arrest and are comatose upon resuscitation. The effects on patient survival are so dramatic that post-cardiac arrest hypothermia is now a standard part of clinical practice guidelines and is also being examined for the protection of the brain after a stroke [263]. Therefore, the use of the hibernating ground squirrel as a model for hibernation research is very practical as it allows us to observe the effects of profound, yet natural, hypothermia $\left(\sim 5^{\circ} \mathrm{C}\right)$ on a mammal.

Hibernating mammals employ a suite of adaptive mechanisms to cope with the harsh environmental conditions of winter that would be harmful or lethal to nonhibernating mammals. As previously mentioned, hibernators survive hypothermia, ischemia-reperfusion, and restricted food resources by upregulating defensive mechanisms to enhance cell preservation and ensure a smooth transition to and from the hypometabolic state. To investigate the protective mechanisms against ischemia-reperfusion, I surveyed targets across a range of cellular processes including the antioxidant response, the DNA damage response, the inflammatory response and the regulation of a known protective mitochondrial peptide in the thirteen-lined ground squirrel (Ictidomys tridecemlineatus). As a result, I identified key targets responsible for the transition out of hibernation such as 
1) the cytoprotective expression of peroxiredoxins, 2) DNA-damage repair proteins, 3) the IRF3 transcription factor and the resulting IFN-induced genes, and 4) a cyto- and neuroprotective mitochondrial peptide. The data show that while global molecular controls suppress many ATP-expensive cellular processes to achieve the hypometabolic state during torpor, arousal from torpor relies on the complex coordination of various cytoprotective strategies including the ones mentioned here. In this capacity, the present thesis has identified the molecular mechanisms that are crucial to successful exit from torpor when animals arouse back to euthermia.

\section{Peroxiredoxins as more than antioxidant enzymes}

Antioxidant enzymes have long been known to be important players in the maintenance of cellular integrity and in combating toxic effects of ROS. However, even ROS have been shown to play important roles such as regulating cell growth, differentiation, progression and cell death, and therefore low concentrations of ROS may be indispensable to cellular signaling and defense. Antioxidants play important roles in the body by preventing oxidant-induced damage to the genome and cell components. They are capable of stabilizing or deactivating free radicals and act by reducing their energy or by giving up their own electrons to stabilize/destroy ROS. Countless studies have examined the beneficial effects of antioxidant enzymes and have linked their functions to various diseases such as aging, cancer, diabetes, Alzheimer's stroke, heart attack and atherosclerosis $[9,51,264,265]$. Therefore, by reducing the exposure to free radicals and consuming antioxidant rich foods, the body's potential for reducing the risk of related morbidities is significantly enhanced. However, when the balance between ROS production and antioxidant defenses is lost, oxidative stress results and the dysregulation of various cellular functions occurs, leading to various pathological conditions.

ROS is a term that encompasses many reactive oxygen containing molecules including free radicals. Types of ROS include the hydroxyl radical, superoxide, hydrogen peroxide, singlet oxygen, nitric oxide, hypochlorite, and several lipid peroxides. Each of these is capable of reacting with membrane lipids, nucleic acids, proteins, enzymes and

other small molecules resulting in extensive cellular damage. ROS are generated in a 
number of ways including (1) as a consequence of aerobic metabolism where $90-95 \%$ of the oxygen utilized by the cell is consumed during mitochondrial respiration, but a small proportion is lost to react with free electrons generating radicals [10]; (2) as oxidative bursts from phagocytes to kill foreign pathogens [266, 267]; and (3) during xenobiotic metabolism [268-270]. Consequently, other activities such as vigorous exercise, chronic inflammation (as in diabetes), infections, illnesses, exposure to allergens and toxins may all contribute to an increase in oxidant load [271-276].

In the present thesis, I describe the expression of PRDX proteins in the liver, BAT and WAT of thirteen-lined ground squirrels (Chapter 2). PRDXs are not only antioxidant enzymes but also play roles in mediating cytoprotective pathways such as: stress-induced death through suppression of JNK signaling, enhancement of NF- $\mathrm{kB}$ expression, regulation of circadian rhythms, protection of cells against DNA damaging agents, preventing infection, imparting immunity as well as regulating tumor growth and metastasis $[27,51$, 96, 120, 177, 277]. The data presented in Chapter 2 revealed that PRDX2, 3, 4 and 6 were significantly enhanced at different time points over the torpor/arousal cycle suggesting that these isoforms are responsive to hibernation in a tissue-specific manner. Although the measurement of overall antioxidant capacity did not reveal significant changes, it can be postulated that this was due to a balancing effect caused by a decrease in the antioxidant activities of other factors. TRX1 was also found to be induced in the liver, BAT and WAT [178] which supports the regulatory mechanism of TRX-dependent PRDX activation. An interesting point was the lack of induction of the antioxidant response during early arousal (aside from PRDX4 in the liver, PRDX2 in the BAT, and PRDX6 in the WAT), a time that would be expected to show an elevated response. It is possible however, that the antioxidant response takes more time to activate, as evidenced by the increase in expression during interbout arousal, or that the maintenance of antioxidant defences at control levels throughout the rest of the cycle is enough to maintain its cytoprotective features. The only PRDX isoform that was consistently found to be suppressed was PRDX1. This isoform does confer antioxidant activity, however a possible reason for its suppression is the redundancy due to PRDX2 and 3. Another possible explanation is that PRDX1 exerts its cytoprotective functions, such as tumor suppression, via the suppression of PTEN/AKT activity [278]. A study examining PRDX1 knockout mice found that they produced more 
cell ROS and died prematurely of Ras-induced cancer. It was found that binding of PRDX1 to PTEN is essential for protecting PTEN from oxidation-induced inactivation and therefore it plays an important role in $\mathrm{H}_{2} \mathrm{O}_{2}$-induced tumour suppression [278]. In fact, this is supported by other work performed in our lab where non-phosphorylated PTEN protein expression and its catalytic activity were elevated during torpor. Therefore, the decrease in PRDX1 that was seen in all tissues could be allowing for the increase in PTEN, as reportedin the skeletal muscle of hibernating ground squirrels [279]. Overall, PRDX activity shows a protective phenotype that is both isoform- and tissue-specific. The ground squirrel appears to employ tight regulatory mechanisms to control the antioxidant response based on the demands of the tissue and assist in the maintenance of viability throughout the torpor-arousal cycle.

Specific Hypothesis 1 revisited: Despite the fact that the expression of PRDXs was not enhanced during arousal, the results suggest that their functions are likely important over the entire process of hibernation, and contribute to other cytoprotective functions that assist the smooth transition in and out of torpor.

\section{DNA repair is "halted" during torpor}

DNA damage occurs in response to endogenous and exogenous agents and initiates a cascading repair response. The ability of a cell to initiate repair is highly dependent on the sensing of damage and the transmission of those signals to repair factors. Understanding the mechanisms that enable cells to sense damage and react appropriately is essential to our understanding of pathologies such as cancer. The mechanics of the repair pathway have long been known and illustrate the initiation of various repair proteins depending on the type of break. Single stranded breaks are quickly repaired before turning into double stranded breaks that, if left unrepaired, may result in gross chromosomal aberrations. Therefore, in order to prevent these potentially lethal effects, cells initiate cell cycle arrest to allow time to repair the damage or induce programmed cell death if the damage is excessive. While the regulation of these pathways is well understood under normal or disease states, the effect of hibernation on the DNA damage repair response is poorly understood. It has long been postulated that because hibernation is a period of 
metabolic rate suppression, that the repair machinery is likely also paused until the return to euthermic conditions. Previous studies have shown that ground squirrels exit from hibernation without any ill effects to their DNA and tissues [207]. Therefore, the aim of the research described in Chapter 3 was to elucidate the regulation of the DNA repair response during hibernation to determine whether the response is in fact globally suppressed or whether damage is in fact accumulating, but being quickly repaired.

As previously mentioned, the cycle of torpor and arousal is marked by periods of ischemia-reperfusion which result in a highly oxidative environment. A well-studied marker of oxidative stress is the oxidation of guanine bases in DNA to produce in 8hydroxy-deoxyguanosine. This marker has been shown to occur in numerous pathologies including cancer, stroke, atherosclerosis, diabetes, neurodegenerative diseases, liver diseases and sleep apnea [39, 140, 149, 150, 280, 281]. Poly-(ADP)ribosyl-polymerase-1 (PARP-1) is a multifaceted enzyme at the center of a wide variety of cellular stress responses. It processes diverse signals and directs cells to specific fates (e.g. repair or cell death). PARP-1 is the most abundant and ubiquitous member of a family of 17 related mammalian proteins [132]. PARP-1 catalyzes the covalent attachment of PAR polymers on itself, the site of the break, as well as acceptor proteins (histones, DNA repair proteins, transcription factors and chromatin modulators) using $\mathrm{NAD}^{+}$as a donor of ADP-ribose units [166]. PARP-1 then recruits many other enzymes to the site of the lesion to initiate repair. Understanding how the repair is initiated in a tissue-specific, or site-specific manner is vital to its medical application as a suppressor of apoptosis during organ transplantation. Hibernation in ground squirrels thus represents a unique opportunity to understand the regulatory mechanisms of DNA repair in a stress-responsive pathway.

In light of this, I chose to study whether DNA damage is in fact occurring during hibernation, by assessing a canonical marker of DNA damage and tracing this control mechanism down to the recruited repair enzymes (Ku80, PARP, Mre11, P95, Rad50, XLF, ATM and ATR). I proposed that the DNA repair response would be differentially regulated at various levels including by post-translational modifications (PTMs) and protein-protein interactions that require very little ATP expenditure. Indeed, the data showed that PARP1 was differentially expressed in a tissue-specific manner. Other PTMs of PARP-1, 
including sumoylation, acetylation, and phosphorylation [282,283] also appear to regulate PARP-1 enzyme activity but, to date, the molecular tools (e,g, specific antibodies) are unavailable since discovery these modifications is very recent.

Of particular interest was the differential regulation of downstream enzymes in the liver and skeletal muscle tissues. The protein expression levels of individual repair enzymes were differentially regulated whereby a significant increase in the sensors (Ku80 and Rad50) was noted in the liver and muscle, respectively, but a decrease in the XLF ligase was noted in both tissues. The only other specific change was a decrease in p-Mre11 in the liver but a strong increase in this target in the muscle. These results suggest that there is likely a steady-state maintenance of binding to sites of damage, but no actual resection of broken ends. Ku80 is a DNA-binding subunit of the DNA-PK complex in NHEJ and is involved in double-stranded break repair [284]. Mice lacking Ku80 have growth dysfunctions, show deficiency in V(D)J recombination and are hypersensitive to gammairradiation [285, 286]. Ku80 knockout cells also exhibit significant repair defects, telomere shortening and chromosomal instability [287]. PARP-1 and Ku80 have high affinity for DNA strand breaks and their interaction is important for genomic stability and other chromatin functions [287]. Therefore, it is likely that the increase in these two factors is as a result of binding to sites of damage to initiate repair, but actual resection does not appear to occur during torpor. Similarly, in the muscle, Rad50 is part of the MRN multi-protein complex composed of Mre11, Rad50 and Nbs1 which assemble at the site of DNA damage and participate in repair [288] analogously to $\mathrm{Ku} 80$.

The suppression in the XLF ligase suggests that binding of sensor enzymes is not suppressed, but ligation or repair of broken ends does not occur during torpor. However, subsequent studies would be required to examine XLF expression during early arousal and interbout arousal to confirm this hypothesis. XLF stimulates ligation repair by interacting with XRCC4 to form filaments of alternating XRCC4 and XLF dimers that bridge DNA ends in vitro[169]. Interestingly, a recent study examined the regulation of XLF in the context of DNA repair and found that AKT-mediated phosphorylation of XLF impairs its NHEJ-repair [170]. Although we did not measure the phosphorylation status of XLF, it is possible that this is an added layer of regulation that permits the reversible control of XLF- 
dependent ligation during a period of hypometabolism. Studies in our lab have shown that AKT is in fact differentially regulated in a tissue specific manner and was found to be induced in the liver and heart of hibernating ground squirrels, but not in the skeletal muscle $[102,171]$. This suggests a possible mechanism for energy savings by suppressing some aspects of DNA repair during a hypometabolic period. During this time, I suspect that the binding of stabilizer enzymes to the sites of the break occurs, but ligation of broken ends is until the arousal period, once body temperature returns to euthermia and normal metabolic functions are reinstated.

In summary, more remains to be explored to further elucidate the regulatory mechanisms of the DNA-damage repair response. The liver appears to succumb to higher levels of damage, but also appears to repair itself in an energy-dependent manner whereas skeletal muscle also appears to be mitigating the damage, but it is also possible that it does not succumb to the same level of stress as the liver. The liver is the central organ that coordinates many aspects of metabolism for the whole body (e.g. nutrient processing, storage of glycogen, gluconeogenesis, ketogenesis (supplying fuel for brain during torpor), processing of nitrogenous wastes for excretion, detoxifying xenobiotics, and more and therefore, stronger protective strategies are employed to protect this tissue. This has been supported in numerous studies including this one and those mentioned early in this thesis where increased antioxidant defenses, anti-apoptosis, and chaperone defenses are induced in liver. By contrast, the skeletal muscle is fairly quiescent during torpor and does not have the same metabolic requirements as the liver. Additionally, skeletal muscle is found closer to the outside of the body than the liver and therefore could be colder than the internal organ. This hypothesis has not been examined in hibernators but it could be a potential explanation for my results due to what is known about hypothermia and decreased metabolic rate. It is plausible to assume that if the skeletal muscle were a couple of degrees colder than the liver, that it could be subjected to lesser oxidative stress, and therefore require less protective mechanisms.

Specific Hypothesis 2 revisited: Although the DNA-damage repair response was differentially regulated between the liver and the skeletal muscle, certain repair factors were in fact enhanced during torpor while others are likely delayed until arousal in order 
to complete repair. Various layers of regulation appear to control these mechanisms including post-translational mechanisms.

\section{Induction of Interferon genes}

The ability of cells to respond to extrinsic and intrinsic signals depends on the transmission of signals through highly ordered signaling pathways. Understanding how these mechanisms provide the cell with the information required to react to a specific signal is essential to our understanding of human health and disease. Several mechanisms have been identified as being activators of various signaling pathways. Phosphorylation by protein kinases and phosphatases is an important regulatory mechanism for the rapid and reversible activation of vital regulatory mechanisms. As well, various ligands such as fatty acids, growth factors, and hormones function to activate signaling pathways in response to a particular signal representative of the metabolic environment. It has been widely accepted that signal transduction and transcription factor activation play crucial roles in the overall health of the cell $[289,290]$ but the molecular mechanisms that connect signaling to response are not well understood in many instances. Therefore, by elucidating these mechanisms, this knowledge will provide valuable information and bridge the gap so that we may determine the components of these signaling pathways, the mechanisms for the coordinated response, and how these signals are transmitted to elicit the desired response.

The immune response relies on both innate and adaptive components mediated by various immune cells including leukocytes that phagocytose, kill pathogens and coordinate additional host responses by releasing inflammatory mediators and cytokines [291]. Once the invading agent is killed, components of the pathogen are presented to $\mathrm{T}$ cells, resulting in activation of the adaptive immune response and the establishment of protective immunity [291]. A key challenge is pathogen discrimination from self and, therefore, the evolution of receptors that recognize conserved motifs have greatly benefited this regulation. Pathogen-associated molecular patterns (PAMPs) are recognized by specific receptors and initiate the immune response. An important receptor family known as the Toll-like receptor (TLR) family has been shown to play an essential role in the activation of innate immunity. TLR signaling occurs through one of two major pathways: the MyD88- 
dependent pathway, which triggers the release of pro-inflammatory cytokines and the Toll/IL-1R domain-containing adaptor-inducing IFN-beta (TRIF)-dependent pathway which also contributes to pro-inflammatory cytokine release, and most importantly, induces type 1 IFN responses. To induce this response, TRIF recruits TRAF3, TBK1 and $\mathrm{IKK} \varepsilon$, which activate IRF3.

In light of this, I chose to identify the activation profile of the IRF3 signaling pathway and trace this down to the IFN genes transcribed by this factor. The upstream regulators of IRF3 were also examined in order to assess how they are regulated during hibernation. Importantly, I proposed that the IFN genes would be expressed in a timespecific manner implying that different stages of the torpor-arousal cycle would be more favourable for the expression of select genes depending on ATP expenditure. In fact, the data showed that the IRF3 transcription factor was subject to differential phosphorylation during hibernation and that its expression was induced in a tissue-specific manner. IRF3 transcript levels were maintained in the liver aside from during torpor, were maintained and then suppressed during arousal in BAT and were induced in WAT during arousal. Relative protein levels revealed a suppression in the liver during arousal, maintenance in BAT, and an induction in WAT during entrance into hibernation. Taken together, these data suggest a tight layer of regulation mediated by protein phosphorylation and perhaps suggest that IRF3 is translocated into the nucleus and initiates transcription of IFN genes during critical periods for specific tissues.

Although most tissues undergo a significant suppression of ATP-expensive processes during torpor, the liver has been shown to remain quite metabolically active, as evidenced by the increase or maintenance in various processes such as translation, apoptosis, autophagy and others mentioned previously. Therefore, it is plausible that IRF3 is phosphorylated, translocates into the nucleus and induces the expression of pro-survival inflammatory agents as a protective mechanism during this crucial time. However, upon arousal, interferon genes are not as necessary since the immune response will be reinitiated on a global level, and ATP supply will again be plentiful. By contrast, brown adipose is well-known to be involved in thermogenesis, a process of rewarming induced during early arousal. The data reveal a maintenance of p-IRF3 expression during early arousal and the 
time-points surrounding it, but a suppression during entrance. This aptly suggests a protective phenotype during the period most critical for this tissue's function [20, 43]. Finally, analysis of white adipose revealed a strong increase in p-IRF3 protein expression during entrance into torpor and a maintenance during early arousal at control levels. Although the WAT is well-known to provide the main source of fuel (triglycerides) during torpor, its role as a key endocrine organ has also been well-established. Therefore, it is postulated that the entrance into torpor stage is a key time-point for the induction of hormones and other factors (possibly including immune cells). Physiological and cellular functions during deep torpor and arousal have been examined in several studies, but our knowledge about entrance into hibernation is more limited. Entrance occurs rather spontaneously and is hard to be predicted, but it is characterized by a decrease in body temperature and a decrease in metabolic function. Several studies in woodchucks, marmots, and squirrels have shown that metabolic rate suppression precedes the development of hypothermia, indicating a temperature-independent depression [292-294]. Several studies from our lab have demonstrated differential regulation of various pathways across the torpor-arousal cycle and have noted an increase in certain ones during entrance such as HSP70 and Nrf2 expression, that were elevated in intestine and heart, respectively $[69,125]$. Other factors have also been measured in WAT but do not show significantly elevated expression during entrance aside from HSP60 [178]. Therefore, further studies into the exact protective mechanisms being employed by WAT during entrance into torpor should be performed. However, another study from our lab measured the levels of AGERAGE in WAT and revealed an increase in RAGE signaling and the downstream transcription factor $n f a t 5$ [180]. Therefore, although IRF3 may not be as significantly implicated for the immune response in WAT, it is possible that other mechanisms are being induced.

Upstream IRF3 inducers were differentially regulated in all tissues. In the liver, most targets were differentially regulated at various time points but could be conferring a method of IRF3 regulation dependent on both control by phosphorylation (by TBK1) and by alternative splicing of targets. Both IKK $\varepsilon$ and TBK1 are subject to alternative splicing and have been shown to regulate IFN gene expression in this way. Conversely, brown adipose showed an increase in several targets during the arousal stages of torpor, namely 
in MAVS and RIG (although not significantly), MDA5, TBK1 and STING. These results support the earlier hypothesis that IRF3 induction occurs during the stages of arousal in order to protect BAT, the tissue whose most critical function is exerted during this time. Finally, targets in WAT were postulated to be induced during entrance, but this theory did not correlate with the results. Three targets, IKKe, MAVS and MDA5 were all found to be significantly upregulated, but mainly during late torpor, and early and interbout arousals. However, TBK1 and STING expression were maintained at control levels. It is possible that the maintenance of TBK1 and IKK $\varepsilon$ expression was sufficient to activate p-IRF3. Incidentally, IRF3 activation has been shown to be site-specific. TBK1 and IKK $\varepsilon$ both phosphorylate IRF3 at site $2\left(\mathrm{Ser}^{396}-\mathrm{Ser}^{405}\right)$ which results in the removal of an autoinhibitory structure to allow interaction with the $\mathrm{CBP} / \mathrm{p} 300$ coactivators. This then facilitates phosphorylation at site $1\left(\operatorname{Ser}^{385}\right.$ and $\left.\operatorname{Ser}^{386}\right)$, required for IRF3 dimerization [295]. Therefore, it is possible that the increase seen in p-IRF3 Ser ${ }^{396}$ is simply the first phosphorylation event and that dimerization and shuttling into the nucleus for transcription has not yet occurred.

Further data are required to confirm the direct regulation between the upstream regulators and IRF3 activation during hibernation, but general correlations can be made based on their expression. In fact, downstream gene expression provides a good picture of IRF3-dependent transcription and provides an overall view of inflammatory activation. In all three tissues, various inflammatory cytokines were found to be induced at different points of the cycle. This suggests that the squirrel is maintaining its inflammatory pathways, but upregulating expression of specific genes during crucial times based on need. It is possible that these immune targets help to confer cytoprotection rather than simply acting as a response to viral infection and help to mitigate tissue damage such as in the case of liver. On the other hand, BAT appears to induce inflammatory gene expression mainly during arousal, suggesting the reactivation of the immune system during a time when there is larger energy availability.

Specific Hypothesis 3 revisited: IRF3 transcription and the expression of its downstream genes was shown to be regulated in a tissue-specific manner. However, inflammation does 
not appear to be suppressed in a global manner in all tissues but, instead, appears to confer cytoprotection in addition to pathogen protection.

\section{S-humanin}

Mitochondria are morphologically distinct from other organelles due to their double-membrane and the presence of the electron transport chain coupled with oxidative phosphorylation used to generate ATP [296]. Although they have a central role in energy transduction, they participate in a variety of other cellular functions including ion homeostasis, intermediary metabolism and apoptosis [57]. However, only a few functions are encoded by their own DNA as most mitochondrial proteins are specified by nuclear genes. Mitochondria have their own genetic system - a genome originating from an endosymbiotic $\alpha$-proteobacterial ancestor. The genetic function of the mitochondrial DNA (mtDNA) is well-conserved being involved in respiration, oxidative phosphorylation, transcription-translation, RNA maturation and protein import [57]. The size of the mitogenome in most eukaryotes is in the range of $15-60 \mathrm{kbp}$, but there are some exceptions[57]. The vertebrate mitochondrial genome is composed of 13 tightly packed protein-coding open reading frames (ORFs), two ribosomal RNAs and multiple tRNA clusters [297]. Transcription of these genes produces large polycistronic RNAs which are processed into distinct coding and non-coding molecules. Transcription also proceeds in an "all or none" fashion, meaning that if one transcript increases, they all increase [297]. The complete mitochondrial genome of the 13-lined ground squirrel was recently sequenced to analyze its gene arrangement [298]. It was determined to be $16,458 \mathrm{bp}$ in length and include the 37 genes typically found in other mammals and was assigned the GenBank accession number KP698974. From this, a portion of the 16S rRNA ORF was identified to contain the sequence analogous to a human mitochondrial peptide known as humanin.

Humanin is an important target of study since it has been confirmed as having a significant role as a neuroprotective peptide in humans. Upon its discovery, it was shown to exhibit strong protective roles in mediating $A \beta$-related pathologies as well as suppress tau plaque formation [226]. At the same time, other studies revealed that it has more than a neuroprotective effect and was also implicated in protection against apoptosis, insulin 
resistance and oxidative stress $[219,225,254]$. This led to the characterization of humanin in other vertebrates including cows, dogs, rats, mice, and other mammals (Szereszewski and Storey, 2019)[74]. This led us to hypothesize as to whether an analogue was present in the 13-lined ground squirrel and whether its expression would be differentially regulated across the torpor-arousal cycle. In this chapter, results revealed that s-humanin, the squirrel-specific analogue of humanin was indeed highly expressed in several tissues including the brain cortex at both the mRNA and protein expression level, as well as at the mRNA transcript level in the adipose tissues (Szereszewski and Storey, 2019).

Sequence homology between humanin, s-humanin and the peptide from other vertebrates revealed supporting evidence for amino-acid functional patterns. Previous studies revealed important amino acids for IGFBP-binding, pro-apoptotic function and for secretion. As well, the amino acid in position 3 was suggested to provide species-specificity as a result of being different in each of the animals analyzed. Data on mRNA and protein expression suggested that s-humanin has both a neuro- and cyto-protective function and likely plays a supporting role in hibernation. Ground squirrel neurons have been found to exhibit remarkable neuroplasticity, therefore s-humanin was suggested to be implicated in this process. Additionally, the adipose tissues are heavily involved in thermogenesis, antioxidant regulation, fatty acid storage and hormonal regulation and therefore are likely to need greater protection from oxidative damage over the torpor-arousal cycle.

Specific Hypothesis 4 revisited: The squirrel analogue of humanin was indeed found to be differentially regulated and was proposed to play a cytoprotective role in protecting key tissues during the transition in and out of hibernation.

\section{Final conclusions}

Oxidative stress is a critical component of the hibernation experience that cannot be avoided. However, hibernating mammals have developed extensive adaptive mechanisms to deal with this stress. The regulation of gene expression, signal transduction pathways, transcription factors, post-translational modifications and mitochondrial peptide secretion all provide the ground squirrel the proper tools to maintain smooth transitions 
from euthermia to torpor to arousal back to euthermia. Despite the global suppression of energy-expensive processes and control mechanisms that characterizes torpor, many pathways remain active, but others, as described in this study, show mechanisms that finetune their response so that pro-survival mechanisms are still maintained, but energyexpensive processes are preferentially suppressed until arousal. In this way, the ground squirrel is able to protect its tissues and macromolecules from damage.

Previous studies have characterized the physiological and molecular adaptations and regulatory mechanisms used by 13-lined ground squirrels (I. tridecemlineatus) and the results from these studies have generated many hypotheses with respect to the animal's responses at the cellular level, which were addressed in this thesis. In Chapter 2, the study of the PRDX isoforms suggested that they are regulating the antioxidant response in a tissue-specific manner during hibernation and in a time-dependent manner. Several PRDXs are induced at different times across the torpor-arousal cycle and are suggested to play roles in processes such as cytoprotection, anti-apoptosis, maintenance of mitochondrial integrity and function as well as some immune system regulatory functions. Chapter $\mathbf{3}$ focused on the DNA damage repair response. The results revealed that damage repair may be in a "holding state" during torpor. That is, although damage still occurs, repair enzymes are shuttled to the location of damage and bind to the site of the lesion, but maintain this holding pattern until conditions become more favourable for resection of broken ends. Specifically, more damage could be occurring in the liver in comparison to the muscle, and this is explained by the significant metabolic demands placed on the liver during the hibernation period. In Chapter 4, the inflammatory response to hibernation was examined at different time points throughout the torpor-arousal cycle with a particular focus on the IRF3 transcription factor and the expression of IFN-regulated genes. The results revealed that IRF3 activation is tissue-dependent where the adipose tissues appear to be most significantly implicated. Expression of downstream IFN genes revealed the strongest response in the brown adipose tissue where several genes were induced during interbout arousal. A specific response was initiated in the white adipose tissue for IFN activation, but not with the specific cytokines examined, and the liver revealed an induction for the recruitment of leukocytes. Therefore, the adipose tissues appear to exhibit the most significant pro-inflammatory response during the arousal period, likely for the recruitment 
of immune targets to sites of infection or as a means of cytoprotection. Results from Chapter 5 revealed the regulatory mechanisms of the squirrel-specific analogue of humanin, s-humanin. This newly identified mitochondrial peptide was shown to have high sequence similarity to the human analogue and revealed tissue-specific regulation during hibernation. Its expression was enhanced particularly in the brain cortex, skeletal muscle and adipose tissues at the gene level, and in the cortex at the protein level. This suggests that the brain, which is actively involved in maintaining neuronal connections and synaptic plasticity during torpor, is employing the use of this peptide as a neuroprotective target by suppressing apoptosis and the immune response as well as by suppressing $A \beta$-related pathologies such as tau plaque formation. 


\section{References}

1. Hermes-Lima M, Zenteno-Savín T (2002) Animal response to drastic changes in oxygen availability and physiological oxidative stress. Comp Biochem Physiol Part C Toxicol Pharmacol 133:537-556. https://doi.org/10.1016/S15320456(02)00080-7

2. Maltepe E, Saugstad OD (2009) Oxygen in Health and Disease: Regulation of Oxygen Homeostasis-Clinical Implications. Pediatr Res 65:261-268. https://doi.org/10.1203/PDR.0b013e31818fc83f

3. Semenza GL (2007) Life with oxygen. Science 318:62-4. https://doi.org/10.1126/science.1147949

4. Mittler R (2002) Oxidative stress, antioxidants and stress tolerance. Trends Plant Sci 7:405-410. https://doi.org/10.1016/S1360-1385(02)02312-9

5. Mittler R (2017) ROS Are Good. Trends Plant Sci 22:11-19. https://doi.org/10.1016/J.TPLANTS.2016.08.002

6. Chang BCD and CJ (2012) Chemistry and biology of reactive oxygen species in signaling or stress responses. Nat Chem Biol 7:504-11. https://doi.org/10.1038/nchembio.607.Chemistry

7. Morin PJ, Storey KB (2007) Antioxidant defense in hibernation: Cloning and expression of peroxiredoxins from hibernating ground squirrels, Spermophilus tridecemlineatus. Arch Biochem Biophys 461:59-65. https://doi.org/10.1016/j.abb.2007.01.035

8. Bandyopadhyay U, Das D, Banerjee, Ranajit K (1999) Reactive Oxygen Species, Oxidative Damage and Cell Death. Curr Sci 77:45-55. https://doi.org/10.1016/b978-0-12-805417-8.00004-4

9. Garbarino VR, Orr ME, Rodriguez KA, Buffenstein R (2015) Mechanisms of oxidative stress resistance in the brain: Lessons learned from hypoxia tolerant extremophilic vertebrates. Arch Biochem Biophys 576:8-16. https://doi.org/10.1016/j.abb.2015.01.029

10. Rao, P. S, Kalva S, Yerramilli A, Mamidi S (2011) Free Radicals and Tissue Damage: Role of Antioxidants. Free Radicals Antioxidants 1:2-7. https://doi.org/10.5530/ax.2011.4.2

11. Andziak B, O'Connor TP, Qi W, et al (2006) High oxidative damage levels in the longest-living rodent, the naked mole-rat. Aging Cell 5:463-471.

https://doi.org/10.1111/j.1474-9726.2006.00237.x

12. Murphy MP (2009) How mitochondria produce reactive oxygen species. Biochem J 417:1-13. https://doi.org/10.1042/BJ20081386

13. Storey K, Storey J (2010) Metabolic rate depression: the biochemistry of mammalian hibernation, Makowski,. Elsevier/Academic Press, Burlington 
14. Ruf T, Geiser F (2015) Daily torpor and hibernation in birds and mammals. Biol Rev 90:891-926. https://doi.org/10.1111/brv.12137

15. Stieler JT, Bullmann T, Kohl F, et al (2011) The physiological link between metabolic rate depression and tau phosphorylation in mammalian hibernation. PLoS One 6:e14530. https://doi.org/10.1371/journal.pone.0014530

16. Dark J (2005) Annual lipid cycles in hibernators: Integration of Physiology and Behavior. Annu Rev Nutr 25:469-497. https://doi.org/10.1146/annurev.nutr.25.050304.092514

17. Carey H V., Andrews MT, Martin SL (2003) Mammalian Hibernation: Cellular and Molecular Responses to Depressed Metabolism and Low Temperature. Physiol Rev 83:1153-1181. https://doi.org/10.1152/physrev.00008.2003

18. Storey KB (2010) Out cold: Biochemical regulation of mammalian hibernation - A mini-review. Gerontology 56:220-230. https://doi.org/10.1159/000228829

19. Wang LCH, Lee T-F (2000) Perspectives on Metabolic Suppression during Mammalian Hibernation and Daily Torpor. In: Life in the Cold. Springer Berlin Heidelberg, Berlin, Heidelberg, pp 149-158

20. Storey KB, Storey JM (2004) Mammalian Hibernation: Biochemical Adaptation and Gene Expression. In: Functional Metabolism. John Wiley \& Sons, Inc., Hoboken, NJ, USA, pp 443-471

21. Geiser F (2004) Metabolic rate and body temperature reduction during hibernation and daily torpor. Annu Rev Physiol 66:239-74.

https://doi.org/10.1146/annurev.physiol.66.032102.115105

22. Storey KB (2003) Mammalian hibernation. Transcriptional and translational controls. Adv Exp Med Biol 543:21-38

23. Storey KB (1996) Metabolic adaptations supporting anoxia tolerance in reptiles: Recent advances. Comp Biochem Physiol - B Biochem Mol Biol 113:23-35. https://doi.org/10.1016/0305-0491(95)02043-8

24. Ighodaro OM, Akinloye OA (2018) First line defence antioxidants-superoxide dismutase (SOD), catalase (CAT) and glutathione peroxidase (GPX): Their fundamental role in the entire antioxidant defence grid. Alexandria J Med 54:287293. https://doi.org/10.1016/J.AJME.2017.09.001

25. Fukai T, Ushio-Fukai M (2011) Superoxide dismutases: role in redox signaling, vascular function, and diseases. Antioxid Redox Signal 15:1583-606. https://doi.org/10.1089/ars.2011.3999

26. Chelikani P, Fita I, Loewen PC (2004) Diversity of structures and properties among catalases. Cell Mol Life Sci 61:192-208. https://doi.org/10.1007/s00018003-3206-5

27. Rhee SG (2016) Overview on Peroxiredoxin. Mol Cells 39:1-5. https://doi.org/10.14348/molcells.2016.2368 
28. Gallagher BM, Phelan SA (2007) Investigating transcriptional regulation of Prdx6 in mouse liver cells. Free Radic Biol Med 42:1270-1277.

https://doi.org/10.1016/j.freeradbiomed.2007.01.023

29. Drechsel DA, Patel M (2010) Respiration-dependent $\mathrm{H} 2 \mathrm{O} 2$ removal in brain mitochondria via the thioredoxin/peroxiredoxin system. J Biol Chem 285:27850 27858. https://doi.org/10.1074/jbc.M110.101196

30. Rhee SG, Kang SW, Chang T-S, et al (2001) Critical Review Peroxiredoxin, a Novel Family of Peroxidases. IUBMB Life 52:35-41

31. Rhee SG, Chang T-S, Bae YS, et al (2003) Cellular regulation by hydrogen peroxide. J Am Soc Nephrol 14:S211-5. https://doi.org/10.1097/01.asn.0000077404.45564.7e

32. Holmgren A (2000) Antioxidant Function of Thioredoxin and Glutaredoxin Systems. Antioxid Redox Signal 2:811-820. https://doi.org/10.1089/ars.2000.2.4811

33. Pannala VR, Dash RK (2015) Mechanistic characterization of the thioredoxin system in the removal of hydrogen peroxide. Free Radic Biol Med 78:42-55. https://doi.org/10.1016/J.FREERADBIOMED.2014.10.508

34. Hampton MB, O'Connor KM (2016) Peroxiredoxins and the Regulation of Cell Death. Mol Cells 39:72-6. https://doi.org/10.14348/molcells.2016.2351

35. Chatterjee N, Walker GC (2017) Mechanisms of DNA damage, repair, and mutagenesis. Environ Mol Mutagen 58:235-263. https://doi.org/10.1002/em.22087

36. Davis AJ, Chen DJ (2013) DNA double strand break repair via non-homologous end-joining. Transl Cancer Res 2:130-143. https://doi.org/10.3978/j.issn.2218676X.2013.04.02

37. Valavanidis A, Vlachogianni T, Fiotakis C (2009) 8-Hydroxy-2' -deoxyguanosine (8-OHdG): A critical biomarker of oxidative stress and carcinogenesis. J Environ Sci Heal - Part C Environ Carcinog Ecotoxicol Rev 27:120-139. https://doi.org/10.1080/10590500902885684

38. Winterbourn CC (2008) Reconciling the chemistry and biology of reactive oxygen species. Nat Chem Biol 4:278-286. https://doi.org/10.1038/nchembio.85

39. Luceri C, Bigagli E, Femia A Pietro, et al (2018) Aging related changes in circulating reactive oxygen species (ROS) and protein carbonyls are indicative of liver oxidative injury. Toxicol reports 5:141-145. https://doi.org/10.1016/j.toxrep.2017.12.017

40. Pang D, Yoo S, Dynan WS, et al (1997) Ku proteins join DNA fragments as shown by atomic force microscopy. Cancer Res 57:1412-5

41. Mari P-O, Florea BI, Persengiev SP, et al (2006) Dynamic assembly of end-joining complexes requires interaction between $\mathrm{Ku} 70 / 80$ and XRCC4. Proc Natl Acad Sci U S A 103:18597-602. https://doi.org/10.1073/pnas.0609061103 
42. Rouble AN, Tessier SN, Storey KB (2014) Characterization of adipocyte stress response pathways during hibernation in thirteen-lined ground squirrels. Mol Cell Biochem 393:271-282. https://doi.org/10.1007/s11010-014-2070-y

43. Yan J, Burman A, Nichols C, et al (2006) Detection of differential gene expression in brown adipose tissue of hibernating arctic ground squirrels with mouse microarrays. Physiol Genomics 25:346-353. https://doi.org/10.1152/physiolgenomics.00260.2005

44. Storey KB, Storey JM (2010) Metabolic rate depression. The biochemistry of mammalian hibernation. Elsevier

45. Logan SM, Luu BE, Storey KB (2016) Turn down genes for WAT? Activation of anti-apoptosis pathways protects white adipose tissue in metabolically depressed thirteen-lined ground squirrels. Mol Cell Biochem 416:47-62.

https://doi.org/10.1007/s11010-016-2695-0

46. Deng Y, Scherer PE (2010) Adipokines as novel biomarkers and regulators of the metabolic syndrome. Ann N Y Acad Sci 1212:E1-E19.

https://doi.org/10.1111/j.1749-6632.2010.05875.x

47. Gómez-Hernández A, Beneit N, Díaz-Castroverde S, Escribano Ó (2016) Differential Role of Adipose Tissues in Obesity and Related Metabolic and Vascular Complications. Int J Endocrinol 2016:1-15. https://doi.org/10.1155/2016/1216783

48. Bjørndal B, Burri L, Staalesen V, et al (2011) Different adipose depots: their role in the development of metabolic syndrome and mitochondrial response to hypolipidemic agents. J Obes 2011:490650. https://doi.org/10.1155/2011/490650

49. Fernández-Sánchez A, Madrigal-Santillán E, Bautista M, et al (2011) Inflammation, Oxidative Stress, and Obesity. Int J Mol Sci 12:3117-3132. https://doi.org/10.3390/ijms12053117

50. Siti HN, Kamisah Y, Kamsiah J (2015) The role of oxidative stress, antioxidants and vascular inflammation in cardiovascular disease (a review). Vascul. Pharmacol. 71:40-56

51. Abbasi A, Corpeleijn E, Gansevoort RT, et al (2014) Circulating peroxiredoxin 4 and type 2 diabetes risk: The Prevention of Renal and Vascular Endstage Disease (PREVEND) study. Diabetologia 57:1842-1849. https://doi.org/10.1007/s00125014-3278-9

52. Esposito K, Ciotola M, Schisano B, et al (2006) Oxidative stress in the metabolic syndrome. J Endocrinol Invest 29:791-795. https://doi.org/10.1007/BF03347372

53. Ozata M, Mergen M, Oktenli C, et al (2002) Increased oxidative stress and hypozincemia in male obesity. Clin Biochem 35:627-631. https://doi.org/10.1016/S0009-9120(02)00363-6

54. Kurtz CC, Lindell SL, Mangino MJ, Carey H V. (2006) Hibernation confers resistance to intestinal ischemia-reperfusion injury. Am J Physiol Liver Physiol 
291:G895-G901. https://doi.org/10.1152/ajpgi.00155.2006

55. Bouma HR, Carey H V., Kroese FGM (2010) Hibernation: the immune system at rest? J Leukoc Biol 88:619-624. https://doi.org/10.1189/jlb.0310174

56. Carey H V., Frank CL, Seifert JP (2000) Hibernation induces oxidative stress and activation of NF- $\mathrm{BB}$ in ground squirrel intestine. J Comp Physiol B Biochem Syst Environ Physiol 170:551-559. https://doi.org/10.1007/s003600000135

57. Burger G, Gray MW, Franz Lang B (2003) Mitochondrial genomes: anything goes. Trends Genet 19:709-716. https://doi.org/10.1016/J.TIG.2003.10.012

58. Nunnari J, Suomalainen A (2012) Mitochondria: In Sickness and in Health. Cell 148:1145-1159. https://doi.org/10.1016/J.CELL.2012.02.035

59. Hashimoto Y, Niikura T, Tajima H, et al (2001) A rescue factor abolishing neuronal cell death by a wide spectrum of familial Alzheimer's disease genes and A. Proc Natl Acad Sci 98:6336-6341. https://doi.org/10.1073/pnas.101133498

60. Hashimoto Y, Ito Y, Niikura T, et al (2001) Mechanisms of neuroprotection by a novel rescue factor humanin from Swedish mutant amyloid precursor protein. Biochem Biophys Res Commun 283:460-8. https://doi.org/10.1006/bbrc.2001.4765

61. Matsuoka M (2015) Protective Effects of Humanin and Calmodulin-Like Skin Protein in Alzheimer's Disease and Broad Range of Abnormalities. Mol Neurobiol 51:1232-1239. https://doi.org/10.1007/s12035-014-8799-1

62. Matsuoka M, Hashimoto Y, Aiso S, Nishimoto I (2006) Humanin and colivelin: Neuronal-death-suppressing peptides for Alzheimer's disease and amyotrophic lateral sclerosis. CNS Drug Rev 12:113-122. https://doi.org/10.1111/j.15273458.2006.00113.x

63. Hashimoto Y, Kurita M, Aiso S, et al (2009) Humanin Inhibits Neuronal Cell Death by Interacting with a Cytokine Receptor Complex or Complexes Involving CNTF Receptor/WSX-1/gp130. Mol Biol Cell 20:2864-2873. https://doi.org/10.1091/mbc.E09-02-0168

64. Yang Z, Zhang Q, Ge J, Tan Z (2008) Protective effects of tetramethylpyrazine on rat retinal cell cultures. Neurochem Int 52:1176-1187. https://doi.org/10.1016/J.NEUINT.2007.12.008

65. Zacharias DG, Kim SG, Massat AE, et al (2012) Humanin, a cytoprotective peptide, is expressed in carotid artherosclerotic plaques in humans. PLoS One 7:e31065. https://doi.org/10.1371/journal.pone.0031065

66. Bjelland S, Seeberg E (2003) Mutagenicity, toxicity and repair of DNA base damage induced by oxidation. Mutat Res Mol Mech Mutagen 531:37-80. https://doi.org/10.1016/J.MRFMMM.2003.07.002

67. Cadet J, Ravanat J-L, TavernaPorro M, et al (2012) Oxidatively generated complex DNA damage: Tandem and clustered lesions. Cancer Lett 327:5-15. 
https://doi.org/10.1016/J.CANLET.2012.04.005

68. Cadet J, Douki T, Ravanat J-L (2010) Oxidatively generated base damage to cellular DNA. Free Radic Biol Med 49:9-21.

https://doi.org/10.1016/J.FREERADBIOMED.2010.03.025

69. Carey H V, Andrews MT, Martin SL (2003) Mammalian hibernation: cellular and molecular responses to depressed metabolism and low temperature. Physiol Rev 83:1153-1181

70. Wei Y, Zhang J, Xu S, et al (2018) Controllable oxidative stress and tissue specificity in major tissues during the torpor-arousal cycle in hibernating Daurian ground squirrels. Open Biol 8:. https://doi.org/10.1098/rsob.180068

71. Ni Z, Storey KB (2010) Heme oxygenase expression and Nrf2 signaling during hibernation in ground squirrelsThis article is one of a selection of papers published in a Special Issue on Oxidative Stress in Health and Disease. Can J Physiol Pharmacol 88:379-387. https://doi.org/10.1139/Y10-017

72. Zhang Y, Storey KB (2018) Life in Suspended Animation: Role of Chaperone Proteins in Vertebrate and Invertebrate Stress Adaptation. In: Regulation of Heat Shock Protein Responses (Asea, A.A.A. and Kaur, P., eds.), Springer I. Springer, Cham, pp 95-137

73. von der Ohe CG, Darian-Smith C, Garner CC, Heller HC (2006) Ubiquitous and Temperature-Dependent Neural Plasticity in Hibernators. J Neurosci 26:1059010598. https://doi.org/10.1523/JNEUROSCI.2874-06.2006

74. Carey H V., Andrews MT, Martin SL (2003) Mammalian Hibernation: Cellular and Molecular Responses to Depressed Metabolism and Low Temperature. Physiol Rev 83:1153-1181. https://doi.org/10.1152/physrev.00008.2003

75. Storey K, Storey (2011) Heat shock proteins and hypometabolism: adaptive strategy for proteome preservation. Res Rep Biol 2:57. https://doi.org/10.2147/rrb.s13351

76. Malik AI, Storey KB (2011) Transcriptional regulation of antioxidant enzymes by FoxO1 under dehydration stress. Gene 485:114-119. https://doi.org/10.1016/j.gene.2011.06.014

77. Bagnyukova T V., Storey KB, Lushchak VI (2005) Adaptive response of antioxidant enzymes to catalase inhibition by aminotriazole in goldfish liver and kidney. Comp Biochem Physiol - B Biochem Mol Biol 142:335-341. https://doi.org/10.1016/j.cbpb.2005.08.003

78. Lushchak VI, Bagnyukova T V., Lushchak O V., et al (2005) Hypoxia and recovery perturb free radical processes and antioxidant potential in common carp (Cyprinus carpio) tissues. Int J Biochem Cell Biol 37:1319-1330.

https://doi.org/10.1016/j.biocel.2005.01.006

79. Willmore WG, Storey KB (1997) Antioxidant systems and anoxia tolerance in a freshwater turtle Trachemys scripta elegans. Mol Cell Biochem 170:177-185. 
https://doi.org/10.1023/A:1006817806010

80. Chainy GBN, Paital B, Dandapat J (2016) An Overview of Seasonal Changes in Oxidative Stress and Antioxidant Defence Parameters in Some Invertebrate and Vertebrate Species. Scientifica (Cairo) 2016:1-8. https://doi.org/10.1155/2016/6126570

81. Wang Y, Branicky R, Noë A, Hekimi S (2018) Superoxide dismutases: Dual roles in controlling ROS damage and regulating ROS signaling. J. Cell Biol. 217:19151928

82. Nita M, Grzybowski A (2016) The Role of the Reactive Oxygen Species and Oxidative Stress in the Pathomechanism of the Age-Related Ocular Diseases and Other Pathologies of the Anterior and Posterior Eye Segments in Adults. Oxid Med Cell Longev 2016:1-23. https://doi.org/10.1155/2016/3164734

83. Kim GH, Kim JE, Rhie SJ, Yoon S (2015) The Role of Oxidative Stress in Neurodegenerative Diseases. Exp Neurobiol 24:325. https://doi.org/10.5607/en.2015.24.4.325

84. Li S, Tan HY, Wang N, et al (2015) The role of oxidative stress and antioxidants in liver diseases. Int. J. Mol. Sci. 16:26087-26124

85. Buzadžić B, Spasić M, Saičić ZS, et al (1990) Antioxidant defenses in the ground squirrel Citellus citellus 2. The effect of hibernation. Free Radic Biol Med 9:407413. https://doi.org/10.1016/0891-5849(90)90017-D

86. Tessier SN, Katzenback BA, Pifferi F, et al (2015) Cytokine and Antioxidant Regulation in the Intestine of the Gray Mouse Lemur (Microcebus murinus) During Torpor. Genomics, Proteomics Bioinforma 13:127-135. https://doi.org/10.1016/j.gpb.2015.03.005

87. Wu CW, Biggar KK, Zhang J, et al (2015) Induction of Antioxidant and Heat Shock Protein Responses During Torpor in the Gray Mouse Lemur, Microcebus murinus. Genomics, Proteomics Bioinforma 13:119-126.

https://doi.org/10.1016/j.gpb.2015.03.004

88. Joanisse DR, Storey KB (1996) Oxidative damage and antioxidants in Rana sylvatica, the freeze-tolerant wood frog. Am J Physiol 271:R545-53. https://doi.org/10.1152/ajpregu.1996.271.3.R545

89. Hermes-Lima M, Storey KB (2017) Relationship between anoxia exposure and antioxidant status in the frog Rana pipiens. Am J Physiol Integr Comp Physiol 271:R918-R925. https://doi.org/10.1152/ajpregu.1996.271.4.r918

90. Nowakowska A, Caputa M, Rogalska J (2011) Defence against oxidative stress in two species of land snails (Helix pomatia and Helix aspersa) subjected to estivation. J Exp Zool Part A Ecol Genet Physiol 315 A:593-601. https://doi.org/10.1002/jez.713

91. Joanisse, Storey (1996) Oxidative stress and antioxidants in overwintering larvae of cold-hardy goldenrod gall insects. J Exp Biol 199:1483-91 
92. Netto LES, Antunes F (2016) The Roles of Peroxiredoxin and Thioredoxin in Hydrogen Peroxide Sensing and in Signal Transduction. Mol Cells 39:65-71. https://doi.org/10.14348/molcells.2016.2349

93. Latimer HR, Veal EA (2016) Peroxiredoxins in Regulation of MAPK Signalling Pathways; Sensors and Barriers to Signal Transduction. Mol Cells 39:40-45. https://doi.org/10.14348/molcells.2016.2327

94. Toledano MB, Huang B (2016) Microbial 2-Cys Peroxiredoxins: Insights into Their Complex Physiological Roles. Mol Cells 39:31-39. https://doi.org/10.14348/molcells.2016.2326

95. Jang HH, Lee KO, Chi YH, et al (2004) Two enzymes in one: Two yeast peroxiredoxins display oxidative stress-dependent switching from a peroxidase to a molecular chaperone function. Cell 117:625-635.

https://doi.org/10.1016/j.cell.2004.05.002

96. O-Neill JS, Reddy AB (2011) Circadian clocks in human red blood cells. Nature 469:498-504. https://doi.org/10.1038/nature09702

97. Furuta T, Imajo-Ohmi S, Fukuda H, et al (2008) Mast cell-mediated immune responses through IgE antibody and Toll-like receptor 4 by malarial peroxiredoxin. Eur J Immunol 38:1341-1350. https://doi.org/10.1002/eji.200738059

98. Kaszubska W, Falls HD, Schaefer VG, et al (2002) Protein tyrosine phosphatase $1 \mathrm{~B}$ negatively regulates leptin signaling in a hypothalamic cell line. Mol Cell Endocrinol 195:109-118. https://doi.org/10.1016/S0303-7207(02)00178-8

99. Irwin ME, Rivera-Del Valle N, Chandra J (2012) Redox Control of Leukemia: From Molecular Mechanisms to Therapeutic Opportunities. Antioxid Redox Signal 18:1349-1383. https://doi.org/10.1089/ars.2011.4258

100. Krapfenbauer K, Engidawork E, Cairns N, et al (2003) Aberrant expression of peroxiredoxin subtypes in neurodegenerative disorders. Brain Res 967:152-160. https://doi.org/10.1016/S0006-8993(02)04243-9

101. Ahsan MK, Lekli I, Ray D, et al (2009) Redox Regulation of Cell Survival by the Thioredoxin Superfamily: An Implication of Redox Gene Therapy in the Heart. Antioxid Redox Signal 11:2741-2758. https://doi.org/10.1089/ars.2009.2683

102. McMullen DC, Hallenbeck JM (2010) Regulation of Akt during torpor in the hibernating ground squirrel, Ictidomys tridecemlineatus. J Comp Physiol B Biochem Syst Environ Physiol 180:927-934. https://doi.org/10.1007/s00360-0100468-8

103. Eaton SL, Roche SL, Llavero Hurtado M, et al (2013) Total Protein Analysis as a Reliable Loading Control for Quantitative Fluorescent Western Blotting. PLoS One 8:e72457. https://doi.org/10.1371/journal.pone.0072457

104. Zhang J, Storey KB (2016) RBioplot: an easy-to-use R pipeline for automated statistical analysis and data visualization in molecular biology and biochemistry. PeerJ 4:e2436. https://doi.org/10.7717/peerj.2436 
105. Muleme HM, Walpole AC, Staples JF (2006) Mitochondrial Metabolism in Hibernation: Metabolic Suppression, Temperature Effects, and Substrate Preferences. Physiol Biochem Zool 79:474-483. https://doi.org/10.1086/501053

106. James R, Staples J, Brown J, et al (2013) The effects of hibernation on the contractile and biochemical properties of skeletal muscles in the thirteen-lined ground squirrel, Ictidomys tridecemlineatus. J Exp Biol 216:2587-94. https://doi.org/10.1242/jeb.080663

107. Baker PJ, Costanzo JP, Lee RE (2007) Oxidative stress and antioxidant capacity of a terrestrially hibernating hatchling turtle. J Comp Physiol B Biochem Syst Environ Physiol 177:875-883. https://doi.org/10.1007/s00360-007-0185-0

108. Page MM, Peters CW, Staples JF, Stuart JA (2009) Intracellular antioxidant enzymes are not globally upregulated during hibernation in the major oxidative tissues of the 13-lined ground squirrel Spermophilus tridecemlineatus. Comp Biochem Physiol - A Mol Integr Physiol 152:115-122. https://doi.org/10.1016/j.cbpa.2008.09.032

109. Wood ZA, Schröder E, Harris JR, Poole LB (2003) Structure, mechanism and regulation of peroxiredoxins. Trends Biochem. Sci. 28:32-40

110. Choi MH, Lee IK, Kim GW, et al (2005) Regulation of PDGF signalling and vascular remodelling by peroxiredoxin II. Nature 435:347-353. https://doi.org/10.1038/nature03587

111. Guyton KZ, Liu Y, Gorospe M, et al (1996) Activation of mitogen-activated protein kinase by $\mathrm{H} 2 \mathrm{O} 2$ : Role in cell survival following oxidant injury. J Biol Chem 271:4138-4142. https://doi.org/10.1074/jbc.271.8.4138

112. MacDonald JA, Storey KB (2005) Mitogen-activated protein kinases and selected downstream targets display organ-specific responses in the hibernating ground squirrel. Int J Biochem Cell Biol 37:679-691.

https://doi.org/10.1016/j.biocel.2004.05.023

113. Xu R, Andres-Mateos E, Mejias R, et al (2013) Hibernating squirrel muscle activates the endurance exercise pathway despite prolonged immobilization. Exp Neurol 247:392-401. https://doi.org/10.1016/j.expneurol.2013.01.005

114. Wonsey DR, Zeller KI, Dang C V (2002) The c-Myc target gene PRDX3 is required for mitochondrial homeostasis and neoplastic transformation. Proc Natl Acad Sci U S A 99:6649-54. https://doi.org/10.1073/pnas.102523299

115. Huh JY, Kim Y, Jeong J, et al (2011) Peroxiredoxin 3 Is a Key Molecule Regulating Adipocyte Oxidative Stress, Mitochondrial Biogenesis, and Adipokine Expression. Antioxid Redox Signal 16:229-243.

https://doi.org/10.1089/ars.2010.3766

116. Zhang J, Guo X, Hamada T, et al (2018) Protective Effects of Peroxiredoxin 4 (PRDX4) on Cholestatic Liver Injury. Int J Mol Sci 19:2509. https://doi.org/10.3390/ijms19092509 
117. Kim TH, Song J, Alcantara Llaguno SR, et al (2012) Suppression of peroxiredoxin 4 in glioblastoma cells increases apoptosis and reduces tumor growth. PLoS One 7:e42818. https://doi.org/10.1371/journal.pone.0042818

118. Rao Z, Wang S, Wang J (2017) Peroxiredoxin 4 inhibits IL-1 $\beta$-induced chondrocyte apoptosis via PI3K/AKT signaling. Biomed Pharmacother 90:414420. https://doi.org/10.1016/j.biopha.2017.03.075

119. Rouble AN, Hefler J, Mamady H, et al (2013) Anti-apoptotic signaling as a cytoprotective mechanism in mammalian hibernation. PeerJ 1:e29. https://doi.org/10.7717/peerj.29

120. Eismann T, Huber N, Shin T, et al (2009) Peroxiredoxin-6 protects against mitochondrial dysfunction and liver injury during ischemia-reperfusion in mice. Am J Physiol Liver Physiol 296:G266-G274. https://doi.org/10.1152/ajpgi.90583.2008

121. Pacifici F, Arriga R, Sorice GP, et al (2014) Peroxiredoxin 6, a novel player in the pathogenesis of diabetes. Diabetes 63:3210-3220. https://doi.org/10.2337/db140144

122. Nagy N, Malik G, Fisher AB, Das DK (2006) Targeted disruption of peroxiredoxin 6 gene renders the heart vulnerable to ischemia-reperfusion injury. Am J Physiol Circ Physiol 291:H2636-H2640. https://doi.org/10.1152/ajpheart.00399.2006

123. Itoh K, Chiba T, Takahashi S, et al (1997) An Nrf2/small Maf heterodimer mediates the induction of phase II detoxifying enzyme genes through antioxidant response elements. Biochem Biophys Res Commun 236:313-322. https://doi.org/10.1006/bbrc.1997.6943

124. Tessier SN, Storey KB (2010) Expression of myocyte enhancer factor-2 and downstream genes in ground squirrel skeletal muscle during hibernation. Mol Cell Biochem 344:151-162. https://doi.org/10.1007/s11010-010-0538-y

125. Morin P, Ni Z, McMullen DC, Storey KB (2008) Expression of Nrf2 and its downstream gene targets in hibernating 13-lined ground squirrels, Spermophilus tridecemlineatus. Mol Cell Biochem 312:121-129. https://doi.org/10.1007/s11010008-9727-3

126. Wang LCH, Wolowyk MW (1988) Torpor in mammals and birds. Can J Zool 66:133-137. https://doi.org/10.1139/z88-017

127. Logan SM, Tessier SN, Tye J, Storey KB (2016) Response of the JAK-STAT pathway to mammalian hibernation in 13-lined ground squirrel striated muscle. Mol Cell Biochem 414:115-127. https://doi.org/10.1007/s11010-016-2665-6

128. Marx JL (1987) Oxygen free radicals linked to many diseases. Science 235:52931. https://doi.org/10.1126/SCIENCE.3810154

129. Yakes FM, Van Houten B (1997) Mitochondrial DNA damage is more extensive and persists longer than nuclear DNA damage in human cells following oxidative 
stress. Proc Natl Acad Sci 94:514-519. https://doi.org/10.1073/pnas.94.2.514

130. Kasai H (1997) Analysis of a form of oxidative DNA damage, 8-hydroxy-2'deoxyguanosine, as a marker of cellular oxidative stress during carcinogenesis. Mutat Res - Rev Mutat Res 387:147-163. https://doi.org/10.1016/S13835742(97)00035-5

131. Wu CW, Storey KB (2014) FoxO3a-mediated activation of stress responsive genes during early torpor in a mammalian hibernator. Mol Cell Biochem 390:185-195. https://doi.org/10.1007/s11010-014-1969-7

132. Kim MY, Zhang T, Kraus WL (2005) Poly(ADP-ribosyl)ation by PARP-1: "PARlaying" NAD+ into a nuclear signal. Genes Dev 19:1951-1967. https://doi.org/10.1101/gad.1331805

133. Jackson SP, Bartek J (2009) The DNA-damage response in human biology and disease. Nature 461:1071-1078. https://doi.org/10.1038/nature08467

134. Ranjha L, Howard SM, Cejka P (2018) Main steps in DNA double-strand break repair: an introduction to homologous recombination and related processes. Chromosoma 1-28. https://doi.org/10.1007/s00412-017-0658-1

135. Gottlieb TM, Jackson SP (1993) The DNA-dependent protein kinase: Requirement for DNA ends and association with Ku antigen. Cell 72:131-142. https://doi.org/10.1016/0092-8674(93)90057-W

136. Paull TT, Gellert M (1998) The $3^{\prime}$ to $5^{\prime}$ exonuclease activity of Mre11 facilitates repair of DNA double-strand breaks. Mol Cell 1:969-979. https://doi.org/10.1016/S1097-2765(00)80097-0

137. Wu CW, Storey KB (2012) Pattern of cellular quiescence over the hibernation cycle in liver of thirteen-lined ground squirrels. Cell Cycle 11:1714-1726. https://doi.org/10.4161/cc.19799

138. Hickson I, Zhao Y, Richardson CJ, et al (2004) Identification and characterization of a novel and specific inhibitor of the ataxia-telangiectasia mutated kinase ATM. Cancer Res 64:9152-9159. https://doi.org/10.1158/0008-5472.CAN-04-2727

139. Burma S, Chen BP, Murphy M, et al (2001) ATM Phosphorylates Histone H2AX in Response to DNA Double-strand Breaks. J Biol Chem 276:42462-42467. https://doi.org/10.1074/jbc.C100466200

140. Lovell MA, Markesbery WR (2007) Oxidative DNA damage in mild cognitive impairment and late-stage Alzheimer's disease. Nucleic Acids Res 35:7497-7504. https://doi.org/10.1093/nar/gkm821

141. Mamady H, Storey KB (2006) Up-regulation of the endoplasmic reticulum molecular chaperone GRP78 during hibernation in thirteen-lined ground squirrels. Mol Cell Biochem 292:89-98. https://doi.org/10.1007/s11010-006-9221-8

142. Tøien Ø, Blake J, Barnes BM (2015) Thermoregulation and energetics in hibernating black bears: metabolic rate and the mystery of multi-day body 
temperature cycles. J Comp Physiol B 185:447-461.

https://doi.org/10.1007/s00360-015-0891-y

143. Gane B, Nandhakumar S, Bhat V, Rao R (2016) Effect of therapeutic hypothermia on chromosomal aberration in perinatal asphyxia. J. Pediatr. Neurosci. 11:25-28

144. Christian E, Zada G, Sung G, Giannotta SL (2008) A review of selective hypothermia in the management of traumatic brain injury. Neurosurg Focus 25:E9. https://doi.org/10.3171/FOC.2008.25.10.E9

145. Ratigan ED, McKay DB (2016) Exploring principles of hibernation for organ preservation. Transplant Rev 30:13-19.

https://doi.org/10.1016/J.TRRE.2015.08.002

146. Storey KB (1996) Oxidative Stress : Animal Adaptations in Nature. Brazilian J Med Biol Res 29:1715-1733

147. Rouble AN, Hefler J, Mamady H, et al (2013) Anti-apoptotic signaling as a cytoprotective mechanism in mammalian hibernation. PeerJ 1:e29.

https://doi.org/10.7717/peerj.29

148. Biggar KK, Storey KB (2015) Low-temperature microRNA expression in the painted turtle, Chrysemys picta during freezing stress. FEBS Lett 589:3665-3670. https://doi.org/10.1016/j.febslet.2015.10.026

149. Wu LL, Chiou C-C, Chang P-Y, Wu JT (2004) Urinary 8-OHdG: a marker of oxidative stress to DNA and a risk factor for cancer, atherosclerosis and diabetics. Clin Chim Acta 339:1-9. https://doi.org/10.1016/J.CCCN.2003.09.010

150. Kono Y, Nakamura K, Kimura H, et al (2006) Elevated Levels of Oxidative DNA Damage in Serum and Myocardium of Patients With Heart Failure. Circ J 70:1001-1005. https://doi.org/10.1253/circj.70.1001

151. Nakajima H, Unoda K-I, Ito T, et al (2012) The Relation of Urinary 8-OHdG, A Marker of Oxidative Stress to DNA, and Clinical Outcomes for Ischemic Stroke. Open Neurol J 6:51-7. https://doi.org/10.2174/1874205X01206010051

152. Ji X, Luo Y, Ling F, et al (2007) Mild hypothermia diminishes oxidative DNA damage and pro-death signaling events after cerebral ischemia: a mechanism for neuroprotection. Front Biosci 12:1737-1747

153. Allan ME, Storey KB (2012) Expression of NF- $\kappa$ B and downstream antioxidant genes in skeletal muscle of hibernating ground squirrels, Spermophilus tridecemlineatus. Cell Biochem Funct 30:166-174. https://doi.org/10.1002/cbf. 1832

154. Biggar KK, Wu C-W, Tessier SN, et al (2015) Modulation of Gene Expression in Key Survival Pathways During Daily Torpor in the Gray Mouse Lemur, Microcebus murinus. Genomics Proteomics Bioinformatics 13:111-118. https://doi.org/10.1016/J.GPB.2015.03.001

155. Krivoruchko A, Storey KB (2010) Forever Young: Mechanisms of Natural Anoxia 
Tolerance and Potential Links to Longevity. Med Cell Longev 3:186-198

156. Maréchal A, Zou L (2013) DNA damage sensing by the ATM and ATR kinases. Cold Spring Harb Perspect Biol 5:a012716.

https://doi.org/10.1101/cshperspect.a012716

157. Blackford AN, Jackson SP (2017) ATM, ATR, and DNA-PK: The Trinity at the Heart of the DNA Damage Response. Mol Cell 66:801-817. https://doi.org/10.1016/J.MOLCEL.2017.05.015

158. Roos WP, Thomas AD, Kaina B (2016) DNA damage and the balance between survival and death in cancer biology. Nat. Rev. Cancer 16:20-33

159. Pan P, Treat MD, van Breukelen F (2014) A systems-level approach to understanding transcriptional regulation by $\mathrm{p} 53$ during mammalian hibernation. J Exp Biol 217:2489-2498. https://doi.org/10.1242/jeb.103614

160. Lee J-H, Paull TT (2004) Direct Activation of the ATM Protein Kinase by the Mre11/Rad50/Nbs1 Complex. Science (80- ) 304:93-96. https://doi.org/10.1126/science.1091496

161. Zou L, Elledge SJ (2003) Sensing DNA damage through ATRIP recognition of RPA-ssDNA complexes. Science (80- ) 300:1542-1548. https://doi.org/10.1126/science. 1083430

162. Luu BE, Wijenayake S, Zhang J, et al (2018) Strategies of biochemical adaptation for hibernation in a South American marsupial, Dromiciops gliroides: 3 . Activation of pro-survival response pathways. Comp Biochem Physiol Part B Biochem Mol Biol 224:26-31. https://doi.org/10.1016/J.CBPB.2017.12.005

163. Wu C-W, Storey KB (2012) Regulation of the mTOR signaling network in hibernating thirteen-lined ground squirrels. J Exp Biol 215:1720-1727. https://doi.org/10.1242/jeb.066225

164. Ronson GE, Piberger AL, Higgs MR, et al (2018) PARP1 and PARP2 stabilise replication forks at base excision repair intermediates through Fbh1-dependent Rad51 regulation. Nat Commun 9:746. https://doi.org/10.1038/s41467-018-031592

165. Bürkle A (2001) PARP-1: A Regulator of Genomic Stability Linked with Mammalian Longevity. ChemBioChem 2:725. https://doi.org/10.1002/14397633(20011001)2:10<725::AID-CBIC725>3.0.CO;2-3

166. D'Amours D, Sallmann FR, Dixit VM, Poirier GG (2001) Gain-of-function of poly(ADP-ribose) polymerase-1 upon cleavage by apoptotic proteases: implications for apoptosis. J Cell Sci 114:3771-3778

167. Goode EL, Ulrich CM, Potter JD (2002) Polymorphisms in DNA Repair Genes and Associations with Cancer Risk. Cancer Epidemiol. Biomarkers Prev. 11:771772

168. Pierce AJ, Hu P, Han M, et al (2001) Ku DNA end-binding protein modulates 
homologous repair of double-strand breaks in mammalian cells. Genes Dev 15:3237-3242. https://doi.org/10.1101/gad.946401

169. Roy S, de Melo AJ, Xu Y, et al (2015) XRCC4/XLF Interaction Is Variably Required for DNA Repair and Is Not Required for Ligase IV Stimulation. Mol Cell Biol 35:3017-3028. https://doi.org/10.1128/mcb.01503-14

170. Liu P, Gan W, Guo C, et al (2015) Akt-Mediated Phosphorylation of XLF Impairs Non-Homologous End-Joining DNA Repair. Mol Cell 57:648-661. https://doi.org/10.1016/J.MOLCEL.2015.01.005

171. Wu C-W, Storey KB (2012) Regulation of the mTOR signaling network in hibernating thirteen-lined ground squirrels. J Exp Biol 215:1720-1727. https://doi.org/10.1242/jeb.066225

172. D'Amours D, Jackson SP (2002) The Mre11 complex: At the crossroads of DNA repair and checkpoint signalling. Nat. Rev. Mol. Cell Biol. 3:317-327

173. Kijas AW, Lim YC, Bolderson E, et al (2015) ATM-dependent phosphorylation of MRE11 controls extent of resection during homology directed repair by signalling through Exonuclease 1. Nucleic Acids Res 43:8352-67. https://doi.org/10.1093/nar/gkv754

174. Lavin MF, Kozlov S, Gatei M, Kijas AW (2015) ATM-dependent phosphorylation of all three members of the MRN complex: From sensor to adaptor. Biomolecules 5:2877-2902

175. Gatei M, Young D, Cerosaletti KM, et al (2000) ATM-dependent phosphorylation of nibrin in response to radiation exposure. Nat Genet 25:115-119. https://doi.org/10.1038/75508

176. Li Z, Li J, Kong Y, et al (2017) Plk1 phosphorylation of Mre11 antagonizes the DNA damage response. Cancer Res 77:3169-3180. https://doi.org/10.1158/00085472.CAN-16-2787

177. Morin PJ, Storey KB (2007) Antioxidant defense in hibernation: Cloning and expression of peroxiredoxins from hibernating ground squirrels, Spermophilus tridecemlineatus. Arch Biochem Biophys 461:59-65. https://doi.org/10.1016/j.abb.2007.01.035

178. Rouble AN, Tessier SN, Storey KB (2014) Characterization of adipocyte stress response pathways during hibernation in thirteen-lined ground squirrels. Mol Cell Biochem 393:271-282. https://doi.org/10.1007/s11010-014-2070-y

179. Logan SM, Tessier SN, Tye J, Storey KB (2016) Response of the JAK-STAT pathway to mammalian hibernation in 13-lined ground squirrel striated muscle. Mol Cell Biochem 414:115-127. https://doi.org/10.1007/s11010-016-2665-6

180. Logan SM, Storey KB (2018) Pro-inflammatory AGE-RAGE signaling is activated during arousal from hibernation in ground squirrel adipose. PeerJ 6:e4911. https://doi.org/10.7717/peerj.4911 
181. Kumari M, Wang X, Lantier L, et al (2016) IRF3 promotes adipose inflammation and insulin resistance and represses browning. J Clin Invest 126:2839-2854. https://doi.org/10.1172/JCI86080

182. Jialal I, Kaur H, Devaraj S (2014) Toll-like Receptor Status in Obesity and Metabolic Syndrome: A Translational Perspective. J Clin Endocrinol Metab 99:39-48. https://doi.org/10.1210/jc.2013-3092

183. Honda K, Takaoka A, Taniguchi T (2006) Type I Inteferon Gene Induction by the Interferon Regulatory Factor Family of Transcription Factors. Immunity 25:349360. https://doi.org/10.1016/J.IMMUNI.2006.08.009

184. Bowie AG, Unterholzner L (2008) Viral evasion and subversion of patternrecognition receptor signalling. Nat Rev Immunol 8:911-922. https://doi.org/10.1038/nri2436

185. Koop A, Lepenies I, Braum O, et al Novel splice variants of human IKKe negatively regulate IKKe-induced IRF3 and NF-kB activation. https://doi.org/10.1002/eji.201040814

186. Wang Z, Ji J, Peng D, et al (2016) Complex Regulation Pattern of IRF3 Activation Revealed by a Novel Dimerization Reporter System. J Immunol 196:4322-30. https://doi.org/10.4049/jimmunol.1502458

187. Biggar KK, Luu BE, Wu CW, et al (2018) Identification of novel and conserved microRNA and their expression in the gray mouse lemur, Microcebus murinus, a primate capable of daily torpor. Gene 677:332-339. https://doi.org/10.1016/J.GENE.2018.08.014

188. Logan SM, Storey KB (2016) Tissue-specific response of carbohydrate-responsive element binding protein (ChREBP) to mammalian hibernation in 13-lined ground squirrels. Cryobiology 73:103-111. https://doi.org/10.1016/j.cryobiol.2016.09.002

189. Dzal Y, McGuire LP, Veselka N, Fenton MB (2011) Going, going, gone: The impact of white-nose syndrome on the summer activity of the little brown bat (Myotis lucifugus). Biol. Lett. 7:392-394

190. Field KA, Johnson JS, Lilley TM, et al (2015) The White-Nose Syndrome Transcriptome: Activation of Anti-fungal Host Responses in Wing Tissue of Hibernating Little Brown Myotis. PLOS Pathog 11:e1005168. https://doi.org/10.1371/journal.ppat.1005168

191. Tanaka Y, Chen ZJ (2012) STING specifies IRF3 phosphorylation by TBK1 in the cytosolic DNA signaling pathway. Sci Signal 5:ra20. https://doi.org/10.1126/scisignal.2002521

192. Iracheta-Vellve A, Petrasek J, Gyongyosi B, et al (2016) Endoplasmic reticulum stress-induced hepatocellular death pathways mediate liver injury and fibrosis via stimulator of interferon genes. J Biol Chem 291:26794-26805. https://doi.org/10.1074/jbc.M116.736991

193. Deng W, Shi M, Han M, et al (2008) Negative regulation of virus-triggered IFN- 
beta signaling pathway by alternative splicing of TBK1. J Biol Chem 283:355907. https://doi.org/10.1074/jbc.M805775200

194. Janssens S, Burns K, Vercammen E, et al (2003) MyD88S, a splice variant of MyD88, differentially modulates NF-kB- and AP-1-dependent gene expression. FEBS Lett 548:103-107

195. Reikine S, Nguyen JB, Modis Y (2014) Pattern Recognition and Signaling Mechanisms of RIG-I and MDA5. Front Immunol 5:342. https://doi.org/10.3389/fimmu.2014.00342

196. Saxena A, Sachin K (2018) A Network Biology Approach for Assessing the Role of Pathologic Adipose Tissues in Insulin Resistance Using Meta-analysis of Microarray Datasets. Curr Genomics 19:630-636. https://doi.org/10.2174/1389202919666180726125645

197. Liu Y, Maekawa T, Yoshida K, et al (2019) The Transcription Factor ATF7 Controls Adipocyte Differentiation and Thermogenic Gene Programming. iScience 13:98-112. https://doi.org/10.1016/J.ISCI.2019.02.013

198. Peters M (1989) Mechanisms of Action of Interferons. Semin Liver Dis 9:

199. Gutterman JU (1994) Cytokine therapeutics: lessons from interferon alpha. Proc Natl Acad Sci U S A 91:1198-205. https://doi.org/10.1073/pnas.91.4.1198

200. Wieser V, Adolph TE, Grander C, et al (2018) Adipose type i interferon signalling protects against metabolic dysfunction. Gut 67:157-165. https://doi.org/10.1136/gutjnl-2016-313155

201. Zhao G-N, Jiang D-S, Li H (2015) Interferon regulatory factors: at the crossroads of immunity, metabolism, and disease. Biochim Biophys Acta - Mol Basis Dis 1852:365-378. https://doi.org/10.1016/J.BBADIS.2014.04.030

202. Prummel MF, Laurberg P (2003) Interferon- $\alpha$ and Autoimmune Thyroid Disease. Thyroid 13:547-551. https://doi.org/10.1089/105072503322238809

203. Hooks JJ, Moutsopoulos HM, Geis SA, et al (1979) Immune Interferon in the Circulation of Patients with Autoimmune Disease. N Engl J Med 301:5-8. https://doi.org/10.1056/NEJM197907053010102

204. Kim KA, Gu W, Lee IA, et al (2012) High Fat Diet-Induced Gut Microbiota Exacerbates Inflammation and Obesity in Mice via the TLR4 Signaling Pathway. PLoS One 7:e47713. https://doi.org/10.1371/journal.pone.0047713

205. Buck LC, Barnes BM (2000) Effects of ambient temperature on metabolic rate, respiratory quotient, and torpor in an arctic hibernator. Am J Physiol Integr Comp Physiol 279:R255-R262

206. Tøien Ø, Drew KL, Chao ML, Rice ME (2001) Ascorbate dynamics and oxygen consumption during arousal from hibernation in Arctic ground squirrels. Am J Physiol Regul Integr Comp Physiol 281:R572-83. https://doi.org/10.1152/ajpregu.2001.281.2.R572 
207. Dave KR, Christian SL, Perez-Pinzon MA, Drew KL (2012) Neuroprotection: Lessons from hibernators. Comp Biochem Physiol - B Biochem Mol Biol 162:1-9. https://doi.org/10.1016/j.cbpb.2012.01.008

208. Lee Y, Johnson KR, Hallenbeck JM (2012) Global Protein Conjugation by Ubiquitin-Like-Modifiers during Ischemic Stress Is Regulated by MicroRNAs and Confers Robust Tolerance to Ischemia. PLoS One 7:e47787. https://doi.org/10.1371/journal.pone.0047787

209. Dujardin S, Colin M, Buée L (2015) Invited review: Animal models of tauopathies and their implications for research/translation into the clinic

210. Arendt T, Stieler J, Holzer M (2015) Brain hypometabolism triggers PHF-like phosphorylation of tau, a major hallmark of Alzheimer's disease pathology. $\mathrm{J}$ Neural Transm 122:531-539. https://doi.org/10.1007/s00702-014-1342-8

211. Butterfield DA, Reed T, Newman SF, Sultana R (2007) Roles of amyloid $\beta$ peptide-associated oxidative stress and brain protein modifications in the pathogenesis of Alzheimer's disease and mild cognitive impairment. Free Radic Biol Med 43:658-677. https://doi.org/10.1016/j.freeradbiomed.2007.05.037

212. Hureau C, Faller P (2009) A $\beta$-mediated ROS production by Cu ions: Structural insights, mechanisms and relevance to Alzheimer's disease. Biochimie 91:12121217. https://doi.org/10.1016/j.biochi.2009.03.013

213. Kirov SA, Petrak LJ, Fiala JC, Harris KM (2004) Dendritic spines disappear with chilling but proliferate excessively upon rewarming of mature hippocampus. Neuroscience 127:69-80. https://doi.org/10.1016/j.neuroscience.2004.04.053

214. Fu Z, Lee SH, Simonetta A, et al (2007) Differential roles of Rap1 and Rap2 small GTPases in neurite retraction and synapse elimination in hippocampal spiny neurons. J Neurochem 100:118-131. https://doi.org/10.1111/j.14714159.2006.04195.x

215. Popov VI, Bocharova LS, Bragin AG (1992) Repeated changes of dendritic morphology in the hippocampus of ground squirrels in the course of hibernation. Neuroscience 48:45-51. https://doi.org/10.1016/0306-4522(92)90336-Z

216. Popov VI, Bocharova LS (1992) Hibernation-induced structural changes in synaptic contacts between mossy fibres and hippocampal pyramidal neurons. Neuroscience 48:53-62. https://doi.org/10.1016/0306-4522(92)90337-2

217. Magarinos AM, McEwen BS, Saboureau M, Pevet P (2006) Rapid and reversible changes in intrahippocampal connectivity during the course of hibernation in European hamsters. Proc Natl Acad Sci 103:18775-18780. https://doi.org/10.1073/pnas.0608785103

218. von der Ohe CG, Darian-Smith C, Garner CC, Heller HC (2006) Ubiquitous and Temperature-Dependent Neural Plasticity in Hibernators. J Neurosci 26:1059010598. https://doi.org/10.1523/JNEUROSCI.2874-06.2006

219. Charununtakorn ST, Shinlapawittayatorn K, Chattipakorn SC, Chattipakorn N 
(2016) Potential Roles of Humanin on Apoptosis in the Heart. Cardiovasc Ther 34:107-114. https://doi.org/10.1111/1755-5922.12168

220. Gong Z, Tas E, Muzumdar R (2014) Humanin and age-related diseases: A new link? Front Endocrinol (Lausanne) 5:210. https://doi.org/10.3389/fendo.2014.00210

221. Guo B, Zhai D, Cabezas E, et al (2003) Humanin peptide suppresses apoptosis by interfering with Bax activation. Nature 423:456-461. https://doi.org/10.1038/nature01627

222. Hashimoto Y, Niikura T, Ito I, et al (2001) Detailed characterization of neuroprotection by a rescue factor humanin against various Alzheimer's diseaserelevant insults. J Neurosci 21:9235-9245. https://doi.org/21/23/9235 [pii]

223. Benaki D, Zikos C, Evangelou A, et al (2005) Solution structure of humanin, a peptide against Alzheimer's disease-related neurotoxicity. Biochem Biophys Res Commun 329:152-160. https://doi.org/10.1016/J.BBRC.2005.01.100

224. Yamagishi Y, Hashimoto Y, Niikura T, Nishimoto I (2003) Identification of essential amino acids in Humanin, a neuroprotective factor against Alzheimer's disease-relevant insults. Peptides 24:585-595. https://doi.org/10.1016/S01969781(03)00106-2

225. Xiao J, Kim SJ, Cohen P, Yen K (2016) Humanin: Functional Interfaces with IGFI. Growth Horm IGF Res 29:21-27. https://doi.org/10.1016/j.ghir.2016.03.005

226. Chai GS, Duan DX, Ma RH, et al (2014) Humanin attenuates Alzheimer-like cognitive deficits and pathological changes induced by amyloid $\beta$-peptide in rats. Neurosci Bull 30:923-935. https://doi.org/10.1007/s12264-014-1479-3

227. Lee C, Yen K, Cohen P (2013) Humanin: A harbinger of mitochondrial-derived peptides? Trends Endocrinol Metab 24:222-228.

https://doi.org/10.1016/j.tem.2013.01.005

228. Xu X, Chua CC, Gao J, et al (2006) Humanin is a novel neuroprotective agent against stroke. Stroke 37:2613-2619.

https://doi.org/10.1161/01.STR.0000242772.94277.1f

229. Cohen P (2014) New role for the mitochondrial peptide humanin: Protective agent against chemotherapy-induced side effects. J Natl Cancer Inst 106:2-3. https://doi.org/10.1093/jnci/dju006

230. Gong Z, Tasset I (2018) Humanin enhances the cellular response to stress by activation of chaperone-mediated autophagy. Oncotarget 9:10832-10833. https://doi.org/10.18632/oncotarget.24396

231. Zhao ST, Huang XT, Zhang C, Ke Y (2012) Humanin protects cortical neurons from ischemia and reperfusion injury by the increased activity of superoxide dismutase. Neurochem Res 37:153-160. https://doi.org/10.1007/s11064-011-05930 
232. Xu X, Chua CC, Gao J, et al (2008) Neuroprotective effect of humanin on cerebral ischemia/reperfusion injury is mediated by a PI3K/Akt pathway. Brain Res 1227:12-18. https://doi.org/10.1016/j.brainres.2008.06.018

233. Kariya S, Hirano M, Nagai Y, et al (2005) Humanin attenuates apoptosis induced by DRPLA proteins with expanded polyglutamine stretches. J Mol Neurosci 25:165-169. https://doi.org/10.1385/JMN:25:2:165

234. Sponne I, Fifre A, Koziel V, et al (2004) Humanin rescues cortical neurons from prion-peptide-induced apoptosis. Mol Cell Neurosci 25:95-102. https://doi.org/10.1016/j.mcn.2003.09.017

235. Hoang PT, Park P, Cobb LJ, et al (2010) The neurosurvival factor Humanin inhibits $\beta$-cell apoptosis via signal transducer and activator of transcription 3 activation and delays and ameliorates diabetes in nonobese diabetic mice. Metabolism 59:343-349. https://doi.org/10.1016/j.metabol.2009.08.001

236. Oh YK, Bachar AR, Zacharias DG, et al (2011) Humanin preserves endothelial function and prevents atherosclerotic plaque progression in hypercholesterolemic ApoE deficient mice. Atherosclerosis 219:65-73. https://doi.org/10.1016/j.atherosclerosis.2011.06.038

237. Muzumdar RH, Huffman DM, Calvert JW, et al (2010) Acute humanin therapy attenuates myocardial ischemia and reperfusion injury in mice. Arterioscler Thromb Vasc Biol 30:1940-1948. https://doi.org/10.1161/ATVBAHA.110.205997

238. Muzumdar RH, Huffman DM, Atzmon G, et al (2009) Humanin: A novel central regulator of peripheral insulin action. PLoS One 4:1-11. https://doi.org/10.1371/journal.pone.0006334

239. Ikonen M, Liu B, Hashimoto Y, et al (2003) Interaction between the Alzheimer's survival peptide humanin and insulin-like growth factor-binding protein 3 regulates cell survival and apoptosis. Proc Natl Acad Sci U S A 100:13042-13047. https://doi.org/10.1073/pnas.2135111100

240. Mamiya T, Ukai M (2001) [Gly(14)]-Humanin improved the learning and memory impairment induced by scopolamine in vivo. Br J Pharmacol 134:1597-9. https://doi.org/10.1038/sj.bjp.0704429

241. Kuliawat R, Klein L, Gong Z, et al (2013) Potent humanin analog increases glucose-stimulated insulin secretion through enhanced metabolism in the cell. FASEB J 27:4890-4898. https://doi.org/10.1096/fj.13-231092

242. Bachar AR, Scheffer L, Schroeder AS, et al (2010) Humanin is expressed in human vascular walls and has a cytoprotective effect against oxidized LDLinduced oxidative stress. Cardiovasc Res 88:360-366.

https://doi.org/10.1093/cvr/cvq191

243. Zhang Y (2008) I-TASSER server for protein 3D structure prediction. In: BMC Bioinformatics. https://bmcbioinformatics.biomedcentral.com/articles/10.1186/1471-2105-9-40. 
Accessed 30 Aug 2017

244. Roy A, Kucukural A, Zhang Y (2010) I-TASSER: A unified platform for automated protein structure and function prediction. Nat Protoc 5:725-738. https://doi.org/10.1038/nprot.2010.5

245. Yang J, Yan R, Roy A, et al (2015) The I-TASSER Suite: protein structure and function prediction. Nat Methods 12:7-8. https://doi.org/10.1038/nmeth.3213

246. Zhang L, Storey KB, Yu D-N, et al (2016) The complete mitochondrial genome of Ictidomys tridecemlineatus (Rodentia: Sciuridae). Mitochondrial DNA 27:26082609. https://doi.org/10.3109/19401736.2015.1041117

247. Bodzioch M, Lapicka-Bodzioch K, Zapala B, et al (2009) Evidence for potential functionality of nuclearly-encoded humanin isoforms. Genomics 94:247-256. https://doi.org/10.1016/j.ygeno.2009.05.006

248. Logan IS (2017) Pseudogenization of the Humanin gene is common in the mitochondrial DNA of many vertebrates. Zool Res 38:198-202.

https://doi.org/10.24272/j.issn.2095-8137.2017.049

249. Zhang L, Huang Y, Storey KB, et al (2016) Complete mitochondrial genomes of Callospermophilus lateralis and Urocitellus richardsonii (Rodentia: Sciuridae). Mitochondrial DNA Part B Resour 1:359-360. https://doi.org/10.1080/23802359.2016.1168716

250. Yu D, Qian K, Storey KB, et al (2015) The complete mitochondrial genome of Nyctalus noctula (Chiroptera: Vespertilionidae). Mitochondrial DNA 00:1-2. https://doi.org/10.3109/19401736.2015.1028032

251. Yu D, Qian K, Storey KB, et al (2016) The complete mitochondrial genome of Myotis lucifugus ( Chiroptera: Vespertilionidae ). 1394:2423-2424.

https://doi.org/10.3109/19401736.2015.1030625

252. Hittel D, Storey KB (2002) The translation state of differentially expressed mRNAs in the hibernating 13-lined ground squirrel (Spermophilus tridecemlineatus). Arch Biochem Biophys 401:244-254. https://doi.org/10.1016/S0003-9861(02)00048-6

253. Eddy SF, Morin P, Storey KB (2005) Cloning and expression of PPAR-gamma and PGC-1alpha from the hibernating ground squirrel, Spermophilus tridecemlineatus. Mol Cell Biochem 269:175-82. https://doi.org/10.1007/s11010005-3459-4

254. Yen K, Lee C, Mehta H, Cohen P (2013) in Stress Resistance. J Mol Endocrinol 50:1-15. https://doi.org/10.1530/JME-12-0203.The

255. Caruso C, Balistreri CR, Candore G (2010) The role of adipose tissue and adipokines in obesity-related inflammatory diseases. Mediators Inflamm 2010:119. https://doi.org/10.1155/2010/802078

256. Harada M, Habata Y, Hosoya M, et al (2004) N-Formylated humanin activates 
both formyl peptide receptor-like 1 and 2. Biochem Biophys Res Commun 324:255-261. https://doi.org/10.1016/j.bbrc.2004.09.046

257. Miller EK (2000) The prefontral cortex and cognitive control. Nat Rev Neurosci 1:59-65. https://doi.org/10.1038/35036228

258. Storey KB, Storey JM (1990) Metabolic Rate Depression and Biochemical Adaptation in Anaerobiosis, Hibernation and Estivation. Q Rev Biol 65:145-174. https://doi.org/10.1086/416717

259. Kloner RA, Bolli R, Marban E, et al (1998) Medical and Cellular Implications of Stunning, Hibernation, and Preconditioning. Circulation 97:1848-1867. https://doi.org/10.1161/01.CIR.97.18.1848

260. Mazzeffi M, Marotta M, Lin H-M, Fischer G (2012) Duration of deep hypothermia during aortic surgery and the risk of perioperative blood transfusion. Ann Card Anaesth 15:266. https://doi.org/10.4103/0971-9784.101855

261. Pacini D, Pantaleo A, Di Marco L, et al (2014) Visceral organ protection in aortic arch surgery: safety of moderate hypothermia. Eur J Cardio-Thoracic Surg 46:438-443. https://doi.org/10.1093/ejcts/ezt665

262. "The Hypothermia after Cardiac Arrest Study Group" (2002) Mild Therapeutic Hypothermia to Improve the Neurologic Outcome after Cardiac Arrest. N Engl J Med 346:549-556. https://doi.org/10.1056/NEJMoa012689

263. Beccaria PF, Turi S, Cristofolini M, et al (2010) Post cardiac arrest therapeutic hypothermia in adult patients, state of art and practical considerations. HSR Proc Intensive Care Cardiovasc Anesth 2:93-103

264. Drew KL, Rice ME, Kuhn TB, Smith MA (2001) Neuroprotective adaptations in hibernation: Therapeutic implications for ischemia-reperfusion, traumatic brain injury and neurodegenerative diseases. Free Radic Biol Med 31:563-573. https://doi.org/10.1016/S0891-5849(01)00628-1

265. Ames BN, Shigenaga MK, Hagen TM (1993) Oxidants, antioxidants, and the degenerative diseases of aging. Proc Natl Acad Sci 90:7915-7922. https://doi.org/10.1073/pnas.90.17.7915

266. Babior BM (2000) Phagocytes and oxidative stress. Am J Med 109:33-44. https://doi.org/10.1016/S0002-9343(00)00481-2

267. Splettstoesser WD, Schuff-Werner P (2002) Oxidative stress in phagocytes \&quot; The enemy within\&quot; Microsc Res Tech 57:441-455. https://doi.org/10.1002/jemt.10098

268. Langen RC., Korn S., Wouters EF. (2003) ROS in the local and systemic pathogenesis of COPD. Free Radic Biol Med 35:226-235. https://doi.org/10.1016/S0891-5849(03)00316-2

269. Eling TE, Curtis JF (1992) Xenobiotic metabolism by prostaglandin H synthase. Pharmacol Ther 53:261-273. https://doi.org/10.1016/0163-7258(92)90012-O 
270. O'Brien PJ (1988) Radical formation during the peroxidase catalyzed metabolism of carcinogens and xenobiotics: The reactivity of these radicals with GSH, DNA, and unsaturated lipid. Free Radic Biol Med 4:169-183. https://doi.org/10.1016/0891-5849(88)90025-1

271. Tsai K, Hsu TG, Hsu KM, et al (2001) Oxidative DNA damage in human peripheral leukocytes induced by massive aerobic exercise. Free Radic Biol Med 31:1465-1472. https://doi.org/10.1016/S0891-5849(01)00729-8

272. Hulgan T, Morrow J, D'Aquila RT, et al (2003) Oxidant Stress Is Increased during Treatment of Human Immunodeficiency Virus Infection. Clin Infect Dis 37:17111717. https://doi.org/10.1086/379776

273. Fernando N, Wickremesinghe S, Niloofa R, et al (2016) Protein Carbonyl as a Biomarker of Oxidative Stress in Severe Leptospirosis, and Its Usefulness in Differentiating Leptospirosis from Dengue Infections. PLoS One 11:e0156085. https://doi.org/10.1371/journal.pone.0156085

274. Sarban S, Kocyigit A, Yazar M, Isikan UE (2005) Plasma total antioxidant capacity, lipid peroxidation, and erythrocyte antioxidant enzyme activities in patients with rheumatoid arthritis and osteoarthritis. Clin Biochem 38:981-986. https://doi.org/10.1016/j.clinbiochem.2005.08.003

275. Marseglia L, Manti S, D'Angelo G, et al (2014) Oxidative Stress in Obesity: A Critical Component in Human Diseases. Int J Mol Sci 16:378-400. https://doi.org/10.3390/ijms16010378

276. Blasiak J, Arabski M, Krupa R, et al (2004) DNA damage and repair in type 2 diabetes mellitus. Mutat Res - Fundam Mol Mech Mutagen 554:297-304. https://doi.org/10.1016/j.mrfmmm.2004.05.011

277. Ishii T, Warabi E, Yanagawa T (2012) Physiological Relevance of Antioxid/Redox Genes; Learning from Genetically Modified Animals Guest Editor: Junichi Fujii Novel roles of peroxiredoxins in inflammation, cancer and innate immunity. J Clin Biochem Nutr 50:91-105. https://doi.org/10.3164/jcbn.11-109

278. Cao J, Schulte J, Knight A, et al (2009) Prdx1 inhibits tumorigenesis via regulating PTEN/AKT activity. EMBO J 28:1505-1517. https://doi.org/10.1038/emboj.2009.101

279. Wu CW, Bell RA, Storey KB (2015) Post-translational regulation of PTEN catalytic function and protein stability in the hibernating 13-lined ground squirrel. Biochim Biophys Acta - Gen Subj 1850:2196-2202. https://doi.org/10.1016/j.bbagen.2015.07.004

280. Snyder B, Shell B, Cunningham JT, Cunningham RL (2017) Chronic intermittent hypoxia induces oxidative stress and inflammation in brain regions associated with early-stage neurodegeneration. Physiol Rep 5:. https://doi.org/10.14814/phy2.13258

281. Nair D, Ramesh V, Gozal D (2012) Adverse cognitive effects of high-fat diet in a 
murine model of sleep apnea are mediated by NADPH oxidase activity.

Neuroscience 227:361-369. https://doi.org/10.1016/j.neuroscience.2012.09.068

282. Ko HL, Ren EC, Ko HL, Ren EC (2012) Functional Aspects of PARP1 in DNA Repair and Transcription. Biomolecules 2:524-548. https://doi.org/10.3390/biom2040524

283. Mangerich A, Bürkle A (2012) Pleiotropic Cellular Functions of PARP1 in Longevity and Aging: Genome Maintenance Meets Inflammation. Oxid Med Cell Longev 2012:1-19. https://doi.org/10.1155/2012/321653

284. Smith GC, Jackson SP (1999) The DNA-dependent protein kinase. Genes Dev 13:916-34. https://doi.org/10.1101/gad.13.8.916

285. Zhu C, Bogue MA, Lim D-S, et al (1996) Ku86-Deficient Mice Exhibit Severe Combined Immunodeficiency and Defective Processing of V(D)J Recombination Intermediates. Cell 86:379-389. https://doi.org/10.1016/S0092-8674(00)80111-7

286. Nussenzweig A, Chen C, da Costa Soares V, et al (1996) Requirement for Ku80 in growth and immunoglobulin V(D)J recombination. Nature 382:551-555. https://doi.org/10.1038/382551a0

287. Tong W-M, Cortes U, Hande MP, et al (2002) Synergistic role of Ku80 and poly(ADP-ribose) polymerase in suppressing chromosomal aberrations and liver cancer formation. Cancer Res 62:6990-6

288. Syed A, Tainer JA (2018) The MRE11-RAD50-NBS1 Complex Conducts the Orchestration of Damage Signaling and Outcomes to Stress in DNA Replication and Repair. Annu Rev Biochem 87:263-294. https://doi.org/10.1146/annurevbiochem-062917-012415

289. Rose BA, Force T, Wang Y (2010) Mitogen-Activated Protein Kinase Signaling in the Heart: Angels Versus Demons in a Heart-Breaking Tale. Physiol Rev 90:15071546. https://doi.org/10.1152/physrev.00054.2009

290. Akazawa H, Komuro I (2003) Roles of Cardiac Transcription Factors in Cardiac Hypertrophy. Circ Res 92:1079-1088. https://doi.org/10.1161/01.RES.0000072977.86706.23

291. Aderem A, Underhill DM (1999) Mechanisms of phagocytosis in macrophages. Annu Rev Immunol 17:593-623. https://doi.org/10.1146/annurev.immunol.17.1.593

292. Heldmaier G.R. (1993) Suppression of metabolic rate in hibernation. Oxford: Westview Press. , Boulder, San Francisco

293. Ortmann S, Heldmaier G (2000) Regulation of body temperature and energy requirements of hibernating Alpine marmots ( Marmota marmota ). Am J Physiol Integr Comp Physiol 278:R698-R704. https://doi.org/10.1152/ajpregu.2000.278.3.R698

294. Heldmaier G, Ortmann S, Elvert R (2004) Natural hypometabolism during 
hibernation and daily torpor in mammals. Respir Physiol Neurobiol 141:317-329. https://doi.org/10.1016/J.RESP.2004.03.014

295. Panne D, McWhirter SM, Maniatis T, Harrison SC (2007) Interferon regulatory factor 3 is regulated by a dual phosphorylation-dependent switch. J Biol Chem 282:22816-22. https://doi.org/10.1074/jbc.M703019200

296. Saraste M (1999) Oxidative phosphorylation at the fin de siècle. Science 283:1488-93. https://doi.org/10.1126/science.283.5407.1488

297. Clayton DA (1991) Replication and Transcription of Vertebrate Mitochondrial DNA. Annu Rev Cell Biol 7:453-478.

https://doi.org/10.1146/annurev.cb.07.110191.002321

298. Zhang L, Storey KB, Yu DN, et al (2016) The complete mitochondrial genome of Ictidomys tridecemlineatus (Rodentia: Sciuridae). Mitochondrial DNA 27:26082609. https://doi.org/10.3109/19401736.2015.1041117 